University of Nebraska - Lincoln

DigitalCommons@University of Nebraska - Lincoln

$12-2008$

\title{
Effective-range theory for an electron in a short-range potential and a laser field
}

M. V. Frolov

Voronezh State University, Russia, frolov@phys.vsu.ru

N. L. Manakov

Voronezh State University, Russia, manakov@phys.vsu.ru

Anthony F. Starace

University of Nebraska-Lincoln, astarace1@unl.edu

Follow this and additional works at: https://digitalcommons.unl.edu/physicsstarace

Part of the Physics Commons

Frolov, M. V.; Manakov, N. L.; and Starace, Anthony F., "Effective-range theory for an electron in a shortrange potential and a laser field" (2008). Anthony F. Starace Publications. 164.

https://digitalcommons.unl.edu/physicsstarace/164

This Article is brought to you for free and open access by the Research Papers in Physics and Astronomy at DigitalCommons@University of Nebraska - Lincoln. It has been accepted for inclusion in Anthony F. Starace Publications by an authorized administrator of DigitalCommons@University of Nebraska - Lincoln. 


\title{
Effective-range theory for an electron in a short-range potential and a laser field
}

\author{
M. V. Frolov ${ }^{*}$ and N. L. Manakov ${ }^{\dagger}$ \\ Department of Physics, Voronezh State University, Voronezh 394006, Russia \\ Anthony F. Starace \\ Department of Physics and Astronomy, The University of Nebraska, Lincoln, Nebraska 68588-0111, USA
}

(Received 12 August 2008; published 19 December 2008)

\begin{abstract}
The time-dependent effective-range (TDER) theory introduced by Frolov et al. [Phys. Rev. Lett. 91, 053003 (2003)] and used in numerous applications for processes involving a linearly polarized intense laser field is presented in detail for the general problem of an electron, initially bound in a short-range potential, interacting with a laser field of arbitrary polarization. The TDER theory combines the well-known effective-range theory for a weakly bound electron in a short-range potential with the quasistationary, quasienergy state (QQES) or Floquet formulation for an electron interacting with a harmonic, time-dependent field, such as a monochromatic laser field. We present the basic underlying assumptions, the relevant boundary conditions, and the key equations for both the quasienergy $\epsilon$ of the system and its associated, three-dimensional, time-dependent wave function $\Phi_{\epsilon}(\mathbf{r}, t)$. The specific equations appropriate for two initial state symmetries (i.e., $s$ - or $p$-valence electrons) and for three cases of laser field polarization (linear, circular, and elliptical) are derived and discussed. Results of the formulation are presented for the specific case of the Stark shift (and level splitting, in the case of an initially degenerate $p$ state) and detachment rates for $\mathrm{H}^{-}, \mathrm{O}^{-}$, the negative alkali-metal ions, and the negative halogen ions.
\end{abstract}

DOI: 10.1103/PhysRevA.78.063418

PACS number(s): 32.80.Rm, 32.80.Gc, 31.15.-p

\section{INTRODUCTION}

The most interesting phenomena resulting from the interaction of an intense laser field with an atomic or molecular system require for their theoretical description not only a nonperturbative treatment of the laser field, but also a proper accounting of interactions of the active electron(s) with the remaining atomic or molecular system. Such phenomena include the well-known "plateaus" in the energy spectra for above-threshold ionization or detachment (ATI or ATD), high harmonic generation (HHG), and laser-assisted electron-atom scattering (LAES); the "knee" in nonsequential double ionization; and the dichroic (i.e., laser field helicity-dependent) effects in the angular distribution of electrons ionized or detached from an atomic system by (or scattered from an atom in the presence of) an elliptically polarized laser field. The "plateau" features and the "knee" may be understood qualitatively in terms of the well-known rescattering scenario $[1,2]$ (see also recent reviews in $[3,4]$ ), in which an ionized or detached electron is driven back to the atomic or molecular system from which it originated. In the case of LAES [5-7], the scattered electron is similarly driven back to the atomic or molecular system from which it was scattered. The dichroic effects in ATI, ATD, and LAES may be understood most simply in terms of the nonzero partialwave phase shifts of an electron moving in the field of an effective atomic potential (see the recent review in [8]). (Note that whereas plateau features usually vanish in strong field processes involving a circularly polarized laser field,

\footnotetext{
*frolov@phys.vsu.ru

†manakov@phys.vsu.ru

$\ddagger$ astarace1@unl.edu
}

they do exist in this case for LAES [7].) Other than for processes involving ionization and/or excitation of more than one electron, a single-active-electron approximation has been found to work well. Within this approximation, the most accurate quantitative descriptions of strong-field phenomena, especially for processes initiated by short laser pulses, involve the direct numerical solution of the time-dependent Schrödinger equation. However, such calculations (i) are often time consuming and hence are most often carried out only for a limited range of laser field parameters, and (ii) the underlying physical origin and interpretation of the results of such calculations are often not transparent and usually require an analysis using simpler theoretical models. Moreover, these models are often specific to the strong-field phenomenon considered. Alternatively, the exact solution of the Schrödinger equation for an approximate model atomic system interacting with a strong laser field offers three main advantages: first, much of the analysis can be done analytically, so that interpretation of the results is often relatively straightforward; second, owing to the less time-consuming numerical requirements, an extensive survey of a strong-field phenomenon over a wide range of laser field parameters is possible; and, third, the existence of such a model system solution on the level of the active electron's wave function permits a unified qualitative description of different strongfield phenomena within the framework of the same theoretical model.

In this paper we give a detailed account of a theory for obtaining the exact solution for such an approximate, singleactive-electron model system interacting with an intense laser field: the time-dependent effective range (TDER) theory. The TDER theory combines two well-established theoretical formulations: the effective range theory (see, e.g., Ref. [9]) for describing a weakly bound electron in a short-range potential that is modeled by two parameters, the scattering 
length and the effective range, and the quasistationary, quasienergy state (QQES) — or Floquet-theory (see, e.g., Ref. [10]) for describing a system interacting with a monochromatic laser field. The TDER theory thus provides a rare opportunity to obtain the exact, three-dimensional solution for a realistic model system (having a single bound state) in which an electron interacts dynamically with both an intense laser field and a model potential, with both of these interactions treated nonperturbatively. A brief report of the TDER theory was presented in 2003 [11], in which the theory was used to analyze the dependence of strong-field ATD phenomena on the initial orbital angular momentum of the active electron. It has since been applied to interpret [12] the first experiment on multiphoton detachment of $\mathrm{F}^{-}$[13], to predict the existence of rescattering effects in the multiphoton regime [14], to analyze the quantum origin of rescattering plateau phenomena in strong field processes [15], to analyze the initial-state symmetry dependence of threshold phenomena in strong-field processes [16], to analyze the initial-state symmetry dependence of HHG rates as well as to benchmark the accuracy of more approximate methods for obtaining HHG rates [17], to predict order-of-magnitude enhancements of LAES yields owing to well-known threshold phenomena [18], and to interpret structures in the variation of harmonic yields with wavelength as due to threshold phenomena [19]. We note that the simpler, single-parameter, zero-range potential (ZRP) model system [20] has been extremely successful in modeling strong-field processes in atoms [21-24] (see also recent reviews in $[4,8,25]$ ). Its main limitation, however, is that it can only describe an active electron bound initially in an $s$ state. This is a significant limitation because a number of studies have shown that strong-field processes are sensitive to the active electron's initial-state symmetry [11,16-19,26,27]. A key motive for the development of the TDER theory is to overcome this inherent limitation of the ZRP model.

Despite our many applications of the TDER theory $[12,14,19]$, a detailed presentation of the theoretical formulation is nevertheless necessary for the following reasons. First, both our initial brief presentation of the TDER theory [11] and its various applications were restricted to the case of a linearly polarized laser field, so that the cases of circular or elliptical laser polarization have not been presented. Second, owing to the magnetic quantum number degeneracy of initial electron states having nonzero orbital angular momentum, a general presentation for this case for an arbitrary laser polarization simply cannot be given. In particular, the choice of an appropriate quantization axis depends on the particular laser polarization being analyzed. These facts govern the presentation of the theory. In this paper we discuss first those general features of the TDER theory that are applicable in all cases. We then present in detail the theory for the simplest case of an electron in an initial $s$ state. Finally, we discuss the case of an electron in an initial state having nonzero orbital angular momentum-namely, an electron initially in a $p$ state. For the latter case, we discuss in turn the specific details necessary for obtaining the complex quasienergy and the QQES wave functions of an initially degenerate bound state for the cases of linear, circular, and elliptic laser polarization. In all cases we illustrate the theory by means of applications to strong-field processes involving negative ions. Since the analysis of plateau features in different strong-field processes requires a more detailed treatment of the QQES wave functions that is specific to each process, in this paper we restrict ourselves to presenting numerical results for effects that are described by the complex quasienergy-i.e., the Stark shift and splitting and the total detachment rates for ground-state negative ions having valence $s$ or $p$ electrons.

More specifically, this paper is organized as follows. In Sec. II we discuss the basic assumptions of the TDER theory, formulate boundary conditions for the QQES wave function at small distances, and discuss the general form of the QQES wave function in the TDER theory for the most general case of an elliptic laser polarization and arbitrary angular momentum $l$ of an electron initially bound in a short-range potential $U(r)$. In Sec. III we apply this general formulation to the case of $l=0$, discuss the connection to the ZRP model, and for the example of the $\mathrm{H}^{-}$ion, provide numerical comparisons of TDER and ZRP results for both the complex quasienergy and certain Fourier coefficients of the time-dependent QQES wave function over a wide range of laser frequencies, intensities, and ellipticities. We also present numerical TDER results for total detachment rates of some negative ions with outer $s$ electrons (namely, $\mathrm{H}^{-}$and the negative alkali-metal ions) for different laser wavelengths and intensities. In Secs. IV-VI we present a detailed analysis of the QQES wave function and the complex quasienergy for an initially degenerate (in the angular momentum quantum number $m$ ) bound $p$ state for the cases of linear (Sec. IV), circular (Sec. V), and elliptic (Sec. VI) laser polarization. While most numerical illustrations of the theory are presented for the $\mathrm{F}^{-}$ion, TDER detachment rates are also presented for other halogen ions and for $\mathrm{O}^{-}$. In Sec. VII we summarize the key results of this paper and present some conclusions. Some mathematical derivations and analyses as well as explicit forms of the matrix elements that enter the TDER equations for the complex quasienergy are presented in three appendixes.

\section{GENERAL FORMULATION}

We consider the quasistationary state of an electron that evolves from an initial bound state $\psi_{0}(\mathbf{r})$ in a spherically symmetric potential $U(r)$ after the adiabatic turn on of a monochromatic laser field having an arbitrary (elliptic) polarization. We require that $U(r)$ vanish for $r \gtrsim r_{c}$ and that it support a shallow bound state $\psi_{0}(\mathbf{r}) \equiv \psi_{\kappa l m}(\mathbf{r})=R_{\kappa l}(r) Y_{l m}(\hat{\mathbf{r}})$, having an energy $E_{0}=-\hbar^{2} \kappa^{2} / 2 m_{e}$, an angular momentum $l$, and an angular momentum projection $m$. By designating the bound state $\psi_{0}(\mathbf{r})$ as "shallow," we mean that $r_{c} \ll \kappa^{-1}$. The initial-state wave function is such that for $r \ll \kappa^{-1} R_{\kappa l}(r) \sim r^{l}$ and for $r \gg \kappa^{-1}$

$$
R_{\kappa l}\left(r \gg \kappa^{-1}\right) \approx C_{\kappa l} r^{-1} \exp (-\kappa r) .
$$

The binding energy $\left|E_{0}\right|$ and the asymptotic coefficient $C_{\kappa l}$ are regarded as parameters of the problem.

Since the photon energy $\hbar \omega$ is assumed to be less than or comparable to the binding energy $\left|E_{0}\right|$, the dipole approximation is appropriate. The electron-photon interaction in length gauge is thus 


$$
V(\mathbf{r}, t)=|e|(\mathbf{r} \cdot \mathbf{F}(t)),
$$

where the electric vector of the laser field is given by

$$
\mathbf{F}(t)=F \operatorname{Re}\left(\mathbf{e} e^{-i \omega t}\right),
$$

where $F$ is the laser field amplitude and $\mathbf{e}$ is its unit photon polarization vector, which is complex for the general case of elliptic polarization: $\left(\mathbf{e} \cdot \mathbf{e}^{*}\right)=1$ and $(\mathbf{e} \cdot \mathbf{k})=0$, where $\mathbf{k}$ $=(\omega / c) \hat{k}$ is the photon wave vector. The degrees of linear $(\ell)$ and circular $(\xi)$ polarization of the laser field, connected to the often-used ellipticity $\eta$ (i.e., the ratio of the minor to major axes of the polarization ellipse), are [28]

$$
\begin{gathered}
\ell=(\mathbf{e} \cdot \mathbf{e})=\mathbf{e}^{2}=\left(1-\eta^{2}\right) /\left(1+\eta^{2}\right), \\
\xi=i\left(\hat{k} \cdot\left[\mathbf{e} \times \mathbf{e}^{*}\right]\right)=2 \eta /\left(1+\eta^{2}\right),
\end{gathered}
$$

where $-1 \leqslant \xi \leqslant+1,0 \leqslant \ell \leqslant 1$, and $\ell^{2}+\xi^{2}=1$. Note that with our definition (3) for $\mathbf{F}(t)$, the laser intensity $I=c F^{2} /(8 \pi)$ does not depend on the polarization.

The exponential (in time) decay of a bound system subjected to a monochromatic perturbation may be described in terms of the complex quasienergy, $\epsilon=\operatorname{Re} \epsilon-i \hbar \Gamma / 2$ (see, e.g., [10]). In this approach, the wave function of a bound electron in the presence of a laser field is sought in the quasienergy (or Floquet) form

$$
\Psi_{\epsilon}(\mathbf{r}, t)=e^{-i \epsilon t / \hbar} \Phi_{\epsilon}(\mathbf{r}, t),
$$

where $\Phi_{\epsilon}(\mathbf{r}, t)$ is periodic in time:

$$
\Phi_{\epsilon}(\mathbf{r}, t)=\sum_{k} \Phi_{k}(\mathbf{r}) e^{-i k \omega t} .
$$

At large distances, the quasienergy harmonics $\Phi_{k}(\mathbf{r})$ behave asymptotically as a superposition of outgoing spherical waves in open detachment channels, $k>n_{0}$ $=\left[\left(|\operatorname{Re} \epsilon|+u_{p}\right) / \hbar \omega\right]$, and decrease exponentially otherwise. (This behavior is the same as for a quasistationary state in time-independent problems [9].) The ponderomotive energy is $u_{p}=e^{2} F^{2} /\left(4 m_{e} \omega^{2}\right)$. Re $\epsilon$ and $\Gamma$ determine the position (i.e., the Stark shift $\left.\Delta \epsilon=\operatorname{Re} \epsilon-E_{0}\right)$ and the total decay rate (i.e., the laser field-induced width $\hbar \Gamma$ ) of the initial bound state $\psi_{0}(\mathbf{r})$. The QQES $\Phi_{\epsilon}(\mathbf{r}, t)$ should be used instead of $\psi_{0}(\mathbf{r})$ for nonperturbative calculations of multiphoton transition amplitudes-e.g., for angular distributions of detached electrons in ATD and for the yields of high-order harmonics in HHG [25,29]. The quasienergy $\epsilon$ is the complex eigenvalue of the "stationary" Schrödinger equation

$$
\left[i \hbar \frac{\partial}{\partial t}+\epsilon+\frac{\hbar^{2}}{2 m_{e}} \Delta-U(r)-V(\mathbf{r}, t)\right] \Phi_{\epsilon}(\mathbf{r}, t)=0
$$

in the space of coordinate-dependent, time-periodic functions; $\epsilon$ is complex owing to the complex asymptotic boundary condition for $\Phi_{\epsilon}(\mathbf{r}, t)$.

\section{A. Boundary condition for the QQES wave function near the origin}

Since the binding potential $U(r)$ vanishes at $r=r_{c}$, the general solution of Eq. (7) for $r>r_{c}$, satisfying outgoing- wave boundary conditions at $r \rightarrow \infty$, is a wave packet of solutions of Eq. (7) with $U(r)=0$-i.e., free-electron states in the laser field. An equation for $\epsilon$ may be obtained by matching this solution to the solution of Eq. (7) inside the well $U(r)$. This task is complicated, in particular, because the perturbation $V(\mathbf{r}, t)$ destroys the spherical symmetry of the problem, but simplifies for a short-range potential well $U(r)$. For this case the actual matching may be replaced by the enforcement of the proper boundary condition for $\Phi_{\epsilon}(\mathbf{r}, t)$ at small $r$. For the case of a shallow bound state of $s$ symmetry, this boundary condition may be formulated at the origin, $r \rightarrow 0$, in accordance with the general ideas of the ZRP model [20-23]. However, for $l>0$, the function $R_{\kappa l}(r)$ vanishes at the origin and thus the boundary condition that replaces Eq. (7) inside the well $U(r)$ must be formulated on a sphere of finite radius (of order $r_{c}$ ). Before formulating this condition for our timedependent problem, we sketch briefly the method developed in Refs. [30,31] for obtaining the quasistationary state $\psi_{E}(\mathbf{r})$ corresponding to the initial state $\psi_{\kappa l m}(\mathbf{r})$ of a weakly bound electron subjected to a long-range static perturbation $V(\mathbf{r})$ - e.g., due to static electric or magnetic fields. The key idea is that the two potentials $U(r)$ and $V(\mathbf{r})$ exert their influence on the electron predominantly in two very different coordinate ranges; i.e., $U(r)$ is important primarily for $r$ $\lesssim r_{c}$, while $V(\mathbf{r})$ is important primarily for $r \gg \kappa^{-1}$. In the region $r_{c} \lesssim r \ll \kappa^{-1}$, the low-energy electron may be considered as virtually free. Thus its wave function may be approximated as a linear superposition of regular and irregular solutions of the field-free equation (7) for the angular momentum $l$ because the potential $U(r)$ binds an electron only in the $|\kappa l m\rangle$ state. As shown in Refs. [30,31] (see also [32]), at distances that are small compared to the "radius" $\kappa^{-1}$ of the bound state $\psi_{\kappa l m}$, the $l$-wave component of $\psi_{E}(\mathbf{r})$ has a universal form that is largely independent of the shape of $U(r)$ :

$$
\int \psi_{E}(\mathbf{r}) Y_{l, m}^{*}(\hat{\mathbf{r}}) d \Omega_{\mathbf{r}} \sim r^{-l-1}+\cdots+B_{l}(E)\left[r^{l}+\cdots\right],
$$

where $B_{l}(E)$ is the ratio of the coefficients of the regular $\left(\sim r^{l}\right)$ and irregular $\left(\sim r^{-l-1}\right)$ solutions of the free-electron Schrödinger equation. The form $(8)$ of $\psi_{E}(\mathbf{r})$ in the $l$-wave channel is consistent with known results for low-energy electron scattering from a potential $U(r)$ [9], in which case $B_{l}(E)$ is expressed in terms of the scattering phase $\delta_{l}(k)$ :

$$
(2 l-1) ! !(2 l+1) ! ! B_{l}(E) \equiv k^{2 l+1} \cot \delta_{l}(k),
$$

where $k=\sqrt{2 m_{e} E} / \hbar$. In accordance with the well-known analyticity arguments common in describing collision problems, the effective-range parametrization for $\delta_{l}(k)$ provides an analytic expression for $B_{l}(E)$ :

$$
(2 l-1) ! !(2 l+1) ! ! B_{l}(E)=-1 / a_{l}+r_{l} k^{2} / 2 .
$$

Since the energy $E$ is complex, this expression for the coefficient $B_{l}(E)$ should be considered as the analytic continuation of the scattering phase $\delta_{l}$ for the case of complex $E$. For the bound- or quasistationary-state problem, the fundamental parameters of the effective-range approach for collision problems, the scattering length $\left(a_{l}\right)$ and the effective range 
$\left(r_{l}\right)$, may be expressed in terms of the binding energy (or $\kappa$ $\left.=\sqrt{2 m_{e}\left|E_{0}\right|} / \hbar\right)$ and the asymptotic coefficient $C_{\kappa l}$ in (1) $[31,33]$ :

$$
\begin{gathered}
(-1)^{l} \kappa^{2 l+1}-a_{l}^{-1}-r_{l} \kappa^{2} / 2=0, \\
(-1)^{l}(2 l+1)-r_{l} \kappa^{-(2 l-1)}=2 \kappa C_{\kappa l}^{-2} .
\end{gathered}
$$

Finally, the boundary condition (8) for $\psi_{E}(\mathbf{r})$ at small $r$ allows one to obtain a transcendental equation for the complex energy $E$ by propagating the $l$-wave part of the appropriate analytic solution of the Schrödinger equation for the potential $V(\mathbf{r})$ to the region of small $r$ and comparing it with Eq. (8).

Returning to Eq. (7), which involves the time-dependent perturbation $V(\mathbf{r}, t)$, it is clear that the ideas of Refs. [30,31] for handling the Schrödinger equation in the case that there are two potentials having significantly different ranges-i.e., $U(r)$ and $V(\mathbf{r}, t)$-are applicable in our case. However, for the QQES, the small- $r$ form (8) should be modified in view of the time dependence of $\Phi_{\epsilon}(\mathbf{r}, t)$. To generalize Eq. (8) for the case of a monochromatic external perturbation, we assume that the $l$-wave component of $\Phi_{\epsilon}(\mathbf{r}, t)$ with a given projection $m$ at small distances involves a time-dependent function $f^{(m)}(t)=\sum_{n} f_{n}^{(m)} e^{-i n \omega t}$. (This is similar to the boundary condition at $r=0$ for the QQES in the ZRP model $[8,23]$.) Although the effects of the potential $V(\mathbf{r}, t)$ are negligible at small distances the derivative $\partial / \partial t$ in Eq. (7) cannot be neglected. In order that the small- $r$ form of $\Phi_{\epsilon}(\mathbf{r}, t)$ satisfy Eq. (7) at small $r \gtrsim r_{c}$ (i.e., in the region $r_{c} \lesssim r \ll \kappa^{-1}$, where both potentials $U(r)$ and $V(\mathbf{r}, t)$ may be omitted), it should involve the quasienergy $\epsilon$ combined with the term $n \hbar \omega$. Specifically, the parameter $E$ in Eq. (10) for the case of a monochromatic perturbation should be replaced by $\mathcal{E}=\epsilon+n \hbar \omega$. Therefore, we postulate the following generalization of the small- $r$ form (8) for the $l$-wave component of the QQES wave function corresponding to the case of a monochromatic, long-range external perturbation $V(\mathbf{r}, t)[11]$ :

$$
\begin{aligned}
\int \Phi_{\epsilon}(\mathbf{r}, t) Y_{l, m}^{*}(\hat{\mathbf{r}}) d \Omega_{\mathbf{r}}= & \sum_{n} \phi_{l, n}(r) e^{-i n \omega t} \sim \sum_{n}\left[r^{-l-1}+\cdots\right. \\
& \left.+B_{l}(\epsilon+n \hbar \omega)\left(r^{l}+\cdots\right)\right] f_{n}^{(m)} e^{-i n \omega t} \\
= & {\left[\frac{1}{r^{l+1}}+\cdots+B_{l}(\epsilon)\left(r^{l}+\cdots\right)\right] f^{(m)}(t) } \\
& +i \frac{(2 l+1)}{(2 l+1) !^{2}} \frac{r_{l} m_{e}}{\hbar} \frac{d}{d t} f^{(m)}(t)\left(r^{l}+\cdots\right),
\end{aligned}
$$

where the parametrization (10) for the coefficient $B_{l}(E)$ remains valid upon substituting $E \rightarrow \mathcal{E}=\epsilon+n \hbar \omega$, and $\phi_{l, n}(r)$ defines the $n$th QQES harmonic in the $l$-wave channel. Note that although Eq. (12) is written only for the $l$-wave component, the exact result for $\Phi_{\epsilon}(\mathbf{r}, t)$ at $r>r_{c}$ (see below) involves all angular momentum components $l^{\prime}$. However, components with $l^{\prime} \neq l$ are laser field induced and thus behave regularly, $\sim r^{l^{\prime}}$, at small $r$. This situation is consistent with the ideas of effective-range theory: since the potential $U(r)$ supports only a bound state of $l$ symmetry, only the scattering phase in the $l$ wave is affected by the interaction of the electron with the binding potential.

Before proceeding, we emphasize the decisive role of the function $f^{(m)}(t)$ for understanding the effects of the binding potential in various strong-laser-field processes and their dependence on the polarization state of the laser field. As shown in Eq. (12), the Fourier coefficients $f_{n}^{(m)}$ determine the population of the $l$-wave components $\phi_{l, n}(r)$ of quasienergy harmonics $\Phi_{n}(\mathbf{r})$ of the QQES $\Phi_{\epsilon}(\mathbf{r}, t)$ in Eq. (6) at small distances $r \leqq r_{c}$. These harmonics appear as a result of absorption $(n>0)$ or stimulated emission $(n<0)$ of $|n|$ photons by an electron having fixed angular momentum $l$ and interacting with the binding potential $U(r)$. Indeed, namely in this channel the electron "feels" the potential $U(r)$ and may absorb and emit photons, thus generating the quasienergy harmonics $\phi_{l, n}(\mathbf{r})$. Therefore, in our approach, all binding potential effects are concentrated in the factors $f_{n}^{(m)}$. Although these coefficients have been introduced initially (at small $r$ ) only in the $l$-wave channel, after matching Eq. (12) with the solution $\Phi_{\epsilon}(\mathbf{r}, t)$ outside the well, they enter the laser fieldinduced angular momentum components of $\Phi_{\epsilon}(\mathbf{r}, t)$ with $l^{\prime}$ $\neq l, \phi_{l^{\prime}, n}$. Thus, they provide the interchannel couplings between the multiphoton channels with different $k$ and $l$ in the QQES wave function in (6). These couplings stem from the effects of the binding potential on the electron in a strong laser field. In our case, these effects occur only in the $l$ wave and are taken into account essentially exactly, thereby affecting all other $l^{\prime} \neq l$ partial waves through interchannel coupling.

The small- $r$ boundary condition (12) corresponds to the case when the magnetic quantum number $m$ remains a conserved quantum number in the presence of an interaction $V(\mathbf{r}, t)$. Otherwise, the right-hand side (RHS) of Eq. (12) involves a sum over all allowed projections $m^{\prime}\left(-l \leqslant m^{\prime} \leqslant l\right)$, with different functions $f^{\left(m^{\prime}\right)}(t)$. As follows from dipole selection rules, the angular momentum $l$ can be conserved only when the electron and laser field exchange an even number of photons, $\Delta n$. Thus only even Fourier coefficients $f_{2 k}^{(m)}$ should be nonzero. For the case of linear polarization, the magnetic quantum number $m$ is conserved in the reference frame with the $z$ axis along the laser polarization direction. Thus there are no additional restrictions on the number $\Delta n$ of exchanged photons, whereas for a circularly polarized laser field, $\Delta n=|\Delta m|$ (in the reference frame with the $z$ axis along the photon wave vector $\mathbf{k})$. Since $|\Delta m| \leqslant 2 l$, for the circular polarization case only a few coefficients $f_{2 k}^{(m)}$ are nonzero for an initial state having an arbitrary angular momentum $l$.

\section{B. General form of the QQES wave function outside the potential well $U(r)$}

Since the small- $r$ form (12) involves an unknown function $f^{(m)}(t)$, this function should be embedded also in the general solution of Eq. (7) outside of the well-i.e., where $U(r)=0$. This solution must thus satisfy the outgoing-wave boundary condition at $r \rightarrow \infty$. Hence it may be expressed in terms of the retarded Green's function of a free electron in a laser field, $G\left(\mathbf{r}, t ; \mathbf{r}^{\prime}=0, t^{\prime}\right)$, multiplied by $f^{(m)}\left(t^{\prime}\right)$ and integrated over $t^{\prime}$. 
In what follows, we define $G$ by the equation

$$
\left(i \hbar \frac{\partial}{\partial t}+\frac{\hbar^{2}}{2 m_{e}} \Delta-V(\mathbf{r}, t)\right) G\left(\mathbf{r}, t ; \mathbf{r}^{\prime}, t^{\prime}\right)=\delta\left(\mathbf{r}-\mathbf{r}^{\prime}\right) \delta\left(t-t^{\prime}\right)
$$

and use the well-known Feynman's form for $G\left(\mathbf{r}, t ; \mathbf{r}^{\prime}, t^{\prime}\right)$,

$$
\begin{aligned}
G\left(\mathbf{r}, t ; \mathbf{r}^{\prime}, t^{\prime}\right) & =-\frac{i}{\hbar}\left[\frac{m_{e}}{2 \pi i \hbar\left(t-t^{\prime}\right)}\right]^{3 / 2} e^{i S\left(\mathbf{r}, t ; \mathbf{r}^{\prime}, t^{\prime}\right) / \hbar} \\
& =G_{0}\left(\mathbf{r}, t ; \mathbf{r}^{\prime}, t^{\prime}\right) e^{i\left[R\left(\mathbf{r}, t ; \mathbf{r}^{\prime}, t^{\prime}\right)+M\left(t, t^{\prime}\right)\right] / \hbar},
\end{aligned}
$$

where $S$ is the classical action for an electron in a laser field and $G_{0}$ is the free-electron Green's function:

$$
\begin{aligned}
& G_{0}\left(\mathbf{r}, t ; \mathbf{r}, t^{\prime}\right)=-\frac{i}{\hbar}\left[\frac{m_{e}}{2 \pi i \hbar\left(t-t^{\prime}\right)}\right]^{3 / 2} \\
& \times \Theta\left(t-t^{\prime}\right) e^{i m_{e}\left(\mathbf{r}-\mathbf{r}^{\prime}\right)^{2} /\left[2\left(t-t^{\prime}\right) \hbar\right],} \\
& R\left(\mathbf{r}, t ; \mathbf{r}^{\prime}, t^{\prime}\right)= \frac{|e|}{\omega^{2}}\left[(\mathbf{r} \cdot \dot{\mathbf{F}}(t))-\left(\mathbf{r}^{\prime} \cdot \dot{\mathbf{F}}\left(t^{\prime}\right)\right)\right. \\
&\left.-\frac{\left(\mathbf{r}-\mathbf{r}^{\prime}\right) \cdot\left(\mathbf{F}(t)-\mathbf{F}\left(t^{\prime}\right)\right)}{t-t^{\prime}}\right], \\
& M\left(t, t^{\prime}\right)=\frac{e^{2}}{2 m_{e} \omega^{4}}\left[\frac{\left[\mathbf{F}(t)-\mathbf{F}\left(t^{\prime}\right)\right]^{2}}{t-t^{\prime}}-\int_{t^{\prime}}^{t}[\dot{\mathbf{F}}(\tau)]^{2} d \tau\right],
\end{aligned}
$$

$\dot{\mathbf{F}}(t) \equiv d \mathbf{F}(t) / d t$, and $\Theta(x)$ is the Heaviside (step) function. In addition, according to Eq. (12), the desired result for $\Phi_{\epsilon}(\mathbf{r}, t)$ should involve the singular terms $\sim r^{-l-1} Y_{l, m}(\hat{\mathbf{r}})$ at $r \rightarrow 0$. Such terms may appear after $l$-fold differentiation of $G\left(\mathbf{r}, t ; \mathbf{r}^{\prime}, t^{\prime}\right)$ over $\mathbf{r}^{\prime}$ followed by an integration over $t^{\prime}$ [involving the function $f^{(m)}\left(t^{\prime}\right)$ ] and, finally, the substitution $\mathbf{r}^{\prime}=0$. As follows from the explicit form of $G$, such differentiation does not change the asymptotic behavior of $\Phi_{\epsilon}(\mathbf{r}, t)$ for $r \rightarrow \infty$. [The idea of differentiating the Green's function in order to obtain the singular solutions for a weakly bound electron with $l>0$ was used first by Demkov and Drukarev [30] for static field potentials $V(\mathbf{r})$ (see also Refs. [31,32]).]

In order to obtain the general form of $\Phi_{\epsilon}(\mathbf{r}, t)$ for $r>r_{c}$, we introduce an auxiliary function

$$
\chi_{\epsilon}\left(\mathbf{r}, \mathbf{r}^{\prime}, t\right)=-\frac{2 \pi \hbar^{2}}{m_{e} \kappa^{l}} \int_{-\infty}^{t} d t^{\prime} e^{i \epsilon\left(t-t^{\prime}\right) / \hbar} f^{(m)}\left(t^{\prime}\right) G\left(\mathbf{r}, t ; \mathbf{r}^{\prime}, t^{\prime}\right),
$$

where $G$ is the retarded Green's function (13) of a free electron interacting with a laser field according to Eq. (2). We employ also the following chain of operator identities involving the $\nabla$ operator:

$$
\begin{aligned}
\mathcal{Y}_{l, m}\left(\nabla_{\mathbf{r}}\right) e^{\alpha r^{2}} & \equiv \sqrt{\frac{(2 l+1) ! !}{4 \pi l !}}\left\{\nabla_{\mathbf{r}}\right\}_{l m} e^{\alpha r^{2}} \\
& =\sqrt{\frac{(2 l+1) ! !}{4 \pi l !}}(2 \alpha)^{l}\{\mathbf{r}\}_{l, m} e^{\alpha r^{2}} \\
& \equiv(2 \alpha r)^{l} e^{\alpha r^{2}} Y_{l, m}(\hat{\mathbf{r}}),
\end{aligned}
$$

where $\mathcal{Y}_{l, m}(\mathbf{a}) \equiv a^{l} Y_{l, m}(\hat{\mathbf{a}})$ is a solid spherical harmonic and $\{a\}_{l, m}$ is the irreducible tensor product of $l$ rank-1 tensors (i.e., $\mathbf{r}$ or $\nabla_{\mathbf{r}}$ ),

$$
\left.\{a\}_{l, m}=\left\{\cdots\{a \otimes a\}_{2} \otimes \cdots a\right\}_{l-1} \otimes a\right\}_{l m},
$$

which is independent of the coupling scheme for the operators $a$ [34]. [In these equations, standard definitions of angular momentum algebra are used [35]; in particular, $Y_{l, m}(\hat{\mathbf{r}})$ $=\sqrt{(2 l+1) ! ! /(4 \pi l !)}\left\{\hat{\mathbf{r}}_{l, m}.\right]$ Taking into account the explicit form of the Green's function $G$ in Eq. (13) and the identities (16), the general form of the QQES $\Phi_{\epsilon}(\mathbf{r}, t)$ corresponding to an initial state of $l$ symmetry may be derived for $r>r_{c}$ as follows:

$$
\begin{aligned}
\Phi_{\epsilon}(\mathbf{r}, t)= & \left.C_{\kappa l} \mathcal{Y}_{l, m}\left(\nabla_{\mathbf{r}^{\prime}}\right) \chi\left(\mathbf{r}, \mathbf{r}^{\prime}, t\right)\right|_{\mathbf{r}^{\prime}=0} \\
= & -C_{\kappa l} \frac{2 \pi \hbar^{2}}{m_{e}}\left(-\frac{i}{\hbar \kappa}\right)^{l} \int_{-\infty}^{t} e^{i \epsilon\left(t-t^{\prime}\right) / \hbar} f^{(m)}\left(t^{\prime}\right) \\
& \times \mathcal{Y}_{l, m}\left(\frac{m_{e} \mathbf{r}}{\left(t-t^{\prime}\right)}+\boldsymbol{\alpha}\left(t, t^{\prime}\right)\right) G\left(\mathbf{r}, t ; 0, t^{\prime}\right) d t^{\prime},
\end{aligned}
$$

where

$$
\boldsymbol{\alpha}\left(t, t^{\prime}\right)=\frac{|e|}{\omega^{2}}\left[\dot{\mathbf{F}}\left(t^{\prime}\right)-\frac{\mathbf{F}(t)-\mathbf{F}\left(t^{\prime}\right)}{t-t^{\prime}}\right] .
$$

As shown in Appendix A, the function (18) satisfies the boundary condition (12) at small $r$ and, for $\mathbf{F}(t)=0$, reduces to the well-known $l$-wave solution of the Schrödinger equation for a free electron of energy $\epsilon$ having the outgoing spherical-wave asymptotic form.

The general procedure for obtaining the equations for $\epsilon$ and $f^{(m)}(t)$ described above varies, depending on the parameters of the problem. In particular, the magnetic quantum number $m$ of the initial state is conserved, as was assumed in the above equations, only in the case of a linearly polarized field whose polarization vector $\mathbf{e}=\mathbf{e}_{z} \equiv \hat{\boldsymbol{\epsilon}}$ defines the $z$ axis For the case of elliptic and circular polarizations, the substates with different $m$ are mixed by the laser field. Thus, in general, the QQES wave function involves a superposition of solutions (18) with different $m$ and different, $m$-dependent functions $f^{(m)}(t)$. In the general case of elliptic polarization, the equations for both the complex quasienergy $\epsilon$ and the functions $f^{(m)}(t)$ become quite cumbersome for initial bound states with high orbital momenta. Thus we restrict our considerations in this paper to the two cases that are most important for applications: $l=0$ ( $s$ state) and $l=1$ ( $p$ state).

For weakly bound electrons, the valence electron's binding energy can vary greatly for different atomic systems, leading to great differences in the rates for strong-field processes. These large differences make it difficult to isolate the effects of other system differences, such as the symmetry of the initial state of the valence electron. In order to better compare results for atomic systems having different binding energies, therefore, we use in what follows scaled units (s.u.), so that each system has a binding energy of 1 s.u. Also, laser field strengths are scaled to the electric field strengths felt by valence electrons in their ground states. 
Specifically, energies and $\omega$ are measured in units of $\left|E_{0}\right|$ and $\left|E_{0}\right| / \hbar$, laser field amplitudes, $F$, in units of $F_{0}$ $=\sqrt{2 m_{e}\left|E_{0}\right|^{3}} /|e| \hbar$, and lengths in units of $\kappa^{-1}$. (However, for the sake of clarity, we shall retain the explicit unit notation $\kappa^{-1}$ when comparing quantities having different length scales.) The scaled unit of intensity, $I_{0}=c F_{0}^{2} /(8 \pi)$, for the $\mathrm{H}^{-}$ and $\mathrm{F}^{-}$ions has the values $I_{0}\left(H^{-}\right)=1.5 \times 10^{12} \mathrm{~W} / \mathrm{cm}^{2}$ and $I_{0}\left(F^{-}\right)=1.37 \times 10^{14} \mathrm{~W} / \mathrm{cm}^{2}$. Thus, the scaled unit of intensity for $\mathrm{H}^{-}\left(\mathrm{F}^{-}\right)$is four (two) orders of magnitude smaller than that for the $\mathrm{H}$ atom: $I_{0}(H)=8.77 \times 10^{15} \mathrm{~W} / \mathrm{cm}^{2}$.

\section{CASE OF AN INITIAL $s$ STATE AND ITS RELATION TO THE ZRP MODEL}

The simplest case is that in which the potential $U(r)$ supports a bound $s$ state having the asymptotic form (1) with coefficient $C_{\kappa l} \equiv C_{0}$. According to Eq. (10) and the small- $r$ boundary condition (12) (with both in s.u.), for proper matching we need the solution of Eq. (7) outside the potential well $U(r)$ that behaves for $r \ll \kappa^{-1}$ as

$$
\left(\frac{1}{r}-\frac{1}{a_{0}}+\frac{1}{2} \epsilon r_{0}\right) f(t)+i \frac{r_{0}}{2} \frac{d f(t)}{d t},
$$

where $f(t) \equiv f^{(m=0)}(t)$. According to Eq. (18), the general form of the QQES wave function outside the well is essentially independent of the shape of $U(r)$ and for $l=0$ becomes (in s.u.)

$$
\Phi_{\epsilon}(\mathbf{r}, t)=-\sqrt{4 \pi} C_{0} \int_{0}^{\infty} d \tau e^{i \epsilon \tau} G(\mathbf{r}, t ; 0, t-\tau) f(t-\tau) .
$$

Expanding the wave function (21) in $r$ [see Eq. (A11)] and comparing the result with the boundary condition (20), one obtains the integro-differential eigenvalue equation for $\epsilon$ and $f(t)$ :

$$
\begin{aligned}
& {\left[\frac{r_{0}}{2}\left(\epsilon-E_{0}\right)-1\right] f(t)+i \frac{r_{0}}{2} \frac{d f(t)}{d t}} \\
& \quad=\frac{1}{\sqrt{4 \pi i}} \int_{0}^{\infty} \frac{d \tau}{\tau^{3 / 2}}\left[e^{i S(t, t-\tau)+i \epsilon \tau} f(t-\tau)-f(t)\right],
\end{aligned}
$$

where $\epsilon-E_{0} \equiv \epsilon+1$, and $S$ is the classical action at the origin, $S\left(t, t^{\prime}\right) \equiv S\left(\mathbf{r}=0, t ; \mathbf{r}^{\prime}=0, t^{\prime}\right)$,

$$
\begin{aligned}
S(t, t-\tau)= & \frac{u_{p}}{\omega}\left[\frac{4 \sin ^{2}(\omega \tau / 2)}{\omega \tau}-\omega \tau-\ell \cos \omega(2 t-\tau)\right. \\
& \left.\times\left(\frac{4 \sin ^{2}(\omega \tau / 2)}{\omega \tau}-\sin \omega \tau\right)\right],
\end{aligned}
$$

where $\ell$ is the degree of linear polarization [cf. Eq. (4)]. In contrast to the case of static perturbations $V(\mathbf{r})$, where the use of the effective-range theory leads to a transcendental equation for the complex energy of the quasistationary state $[30,31]$, the resulting equations for the complex quasienergy of the QQES are more complicated. We have found that the most efficient way to solve the eigenvalue equation (22) is to convert it to a linear system of algebraic equations for the
Fourier coefficients $f_{n}$ of $f(t)$. As was argued qualitatively in Sec. II, only even coefficients $f_{n=2 k}$ are nonzero, as follows also directly from the explicit form of $S(t, t-\tau)$.

Taking into account the effective-range relations (11), the parameters $a_{0}$ and $r_{0}$ in Eq. (22) may be expressed in terms of $\kappa$ and $C_{0}$ as follows:

$$
1 / a_{0}=\kappa\left(1-\kappa r_{0} / 2\right), \quad \kappa r_{0}=1-2 \kappa C_{0}^{-2} .
$$

Generally, the effective-range expansions, like Eq. (10), assume that the parameter $r_{0}$ is of the order of $r_{c}$-i.e., the effective range of the potential $U(r)$. Thus, for a shallow bound state, the parameter $\kappa r_{0}$ is small. In the lowest-order approximation (assuming $\kappa r_{0} \ll 1$ ), one may neglect $r_{0}$ in Eq. (24), yielding $a_{0}=\kappa^{-1}$ and $C_{0} \equiv C_{0}^{z r p}=\sqrt{2 \kappa}$. (This approximation corresponds to the scattering length approximation in collision problems [9].) In this case, the empirical parameter $r_{0}$ (or, equivalently, $C_{0}$ ) does not affect the results for $\epsilon$ and $f(t)$ and the results in Eqs. (21) and (22) coincide with those in the ZRP model $[8,23]$. This fact has a simple explanation, taking into account that Eq. (20) at $r_{0}=0$ reduces to the following:

$$
\left(r^{-1}-\kappa\right) f(t) .
$$

Since $r_{0} \sim r_{c}$ [36], the approximation $r_{0}=0$ means that the boundary condition (25) may be formulated directly at the origin, as is done in the QQES approach for the ZRP model [23]. Thus, for the case of the ZRP model, the QQES wave function (21) is valid at any $r$ and may be normalized using the proper normalization condition for the QQESs [25].

The TDER theory results involving both parameters $\kappa$ and $r_{0}$ allow one to account more precisely for the specifics of particular negative ions because they contain a next-order correction to the ZRP result. First, they give the effectiverange-based justification for introducing the proper asymptotic factor $C_{0}$ for the QQES wave function (21) when applying the ZRP results to real ions. [For the case of onephoton detachment of $\mathrm{H}^{-}$, similar arguments were used in Ref. [37] (see also [38]).] Second, in the TDER theory the effective radius $r_{0}$ enters Eq. (22) for $\epsilon$ and $f(t)$, thus giving corrections beyond the ZRP model to these important parameters. The role of these corrections becomes clear after expanding $f(t)$ in even harmonics of the laser field and rewriting Eq. (22) as a matrix equation

$$
\zeta(\epsilon, 2 k \omega) f_{2 k}=\sum_{k^{\prime}=-\infty}^{\infty} M_{k, k^{\prime}}(\epsilon) f_{2 k^{\prime}},
$$

where

$$
\zeta(\epsilon, 2 k \omega)=\frac{r_{0}}{2}(\epsilon+1+2 k \omega)-1-i \sqrt{\epsilon+2 k \omega},
$$

and the explicit form of $M_{k, k^{\prime}}(\epsilon)$ in terms of one-dimensional integrals of Bessel functions is given in Appendix B. Note that in deriving the matrix equation (26) from Eq. (22) the diagonal matrix elements that originate from the integral on the RHS of Eq. (22) have both field-independent and fielddependent parts. The term $i \sqrt{\epsilon+2 k \omega}$ on the RHS of Eq. (27) is the field-independent part of these matrix elements, so that in our final form (26) of the matrix equation each matrix 
TABLE I. Parameters for negative ions with $s$-state outer electrons; $\mathcal{R}_{0}=\left[C_{0} / C_{0}^{(Z R P)}\right]^{2}$, where $C_{0}^{(Z R P)}=\sqrt{2 \kappa}$.

\begin{tabular}{lccccccc}
\hline \hline Ion & $\left|E_{0}\right|(\mathrm{eV})$ & $\kappa($ a.u. $)$ & $C_{0}($ a.u $)$ & $C_{0}$ (s.u.) & $\mathcal{R}_{0}$ & $a_{0} \kappa$ & $r_{0} \kappa$ \\
\hline $\mathrm{H}^{-}$ & 0.755 & 0.236 & 1.12 & 2.304 & 2.65 & 1.453 \\
$\mathrm{Li}^{-}$ & 0.612 & 0.212 & 1 & 2.172 & 2.36 & 1.405 \\
$\mathrm{Na}^{-}$ & 0.550 & 0.201 & 1 & 2.231 & 2.49 & 1.427 & 0.623 \\
$\mathrm{~K}^{-}$ & 0.502 & 0.192 & 0.9 & 2.054 & 2.11 & 1.357 \\
$\mathrm{Rb}^{-}$ & 0.486 & 0.189 & 0.8 & 1.840 & 1.69 & 1.257 \\
\hline \hline
\end{tabular}

element $M_{k, k^{\prime}}$ is field dependent and vanishes in the absence of a laser field. The Fredholm determinant of the system (26) gives a transcendental equation for the complex quasienergy:

$$
\operatorname{det}\left|\zeta(\epsilon, 2 k \omega) \delta_{k, k^{\prime}}-M_{k, k^{\prime}}(\epsilon)\right|=0,
$$

with the boundary condition $\epsilon=E_{0} \equiv-1$ at $F=0$.

Generally, the term involving $r_{0}$ on the left-hand side (LHS) of Eq. (26) influences the numerical results for $\epsilon$ in both the perturbative and strong-field regimes. In the perturbative regime, the role of terms $\sim r_{0}$ is clear from the Brillouin-Wigner expansion of Eq. (28):

$$
\begin{aligned}
\zeta(\epsilon ; 0)= & M_{0,0}(\epsilon)+\sum_{k \neq 0} \frac{M_{0, k}(\epsilon) M_{k, 0}(\epsilon)}{\zeta(\epsilon ; 2 k \omega)-M_{k, k}(\epsilon)} \\
& +\sum_{k \neq k^{\prime}} \frac{M_{0, k}(\epsilon) M_{k, k^{\prime}}(\epsilon) M_{k^{\prime}, 0}(\epsilon)}{\left[\zeta(\epsilon ; 2 k \omega)-M_{k, k}(\epsilon)\right]\left[\zeta\left(\epsilon ; 2 k^{\prime} \omega\right)-M_{k^{\prime}, k^{\prime}}(\epsilon)\right]} \\
& +\cdots,
\end{aligned}
$$

where $r_{0}$-dependent terms (together with $\epsilon$ ) enter the denominators on the RHS of this equation. Thus, after further expansion of $\epsilon$ in $F$ in order to obtain the standard RayleighSchrödinger expansion for $\epsilon$ and $f_{2 k}$, these expansions will involve a power dependence on $\kappa r_{0}$.

The eigenvalue equation (22) is valid for an arbitrary elliptic polarization of the laser field. For the case of circular polarization $(\ell=0)$, the kernel of the integral on the RHS of Eq. (22) is independent of $t$ [see Eq. (23)], so that the dependence of $f(t)$ on $t$ vanishes and (22) reduces to a transcendental equation for $\epsilon$ :

$$
\begin{aligned}
& \frac{1}{2} r_{0}\left(\epsilon-E_{0}\right)-1=\frac{1}{\sqrt{4 \pi i}} \int_{0}^{\infty} \frac{d \tau}{\tau^{3 / 2}} \\
& \left.\times \exp \left(i\left[\frac{4 u_{p}}{\omega} \frac{\sin ^{2} \frac{\omega \tau}{2}}{\omega \tau}+\left(\epsilon-u_{p}\right) \tau\right]\right)-1\right\} \text {. }
\end{aligned}
$$

This result coincides with that obtained in Ref. [31] (see also [39]). At $r_{0}=0$, it reduces to the result in Refs. [21,22] for a ZRP model. [In Refs. [21,31] the authors solved the problem in the reference frame rotating with the field frequency $\omega$, in which case Eq. (7) becomes time independent.] Note also that for $k \neq k^{\prime}$, the matrix elements $M_{k, k^{\prime}}(\epsilon)$ vanish for the case of circular polarization (i.e., $\xi= \pm 1$ ), so that only the term $M_{0,0}(\epsilon)$ in Eq. (29) contributes. For this reason, the results for circular polarization are least sensitive to the value of $r_{0}$.

The complete characterization of the QQES wave function (21) for a particular negative ion requires two parameters $E_{0}$ (or $\left.\kappa\right)$ and $C_{0}$ and the solution of Eqs. (28) and (26) for the quasienergy $\epsilon$ and the coefficients $f_{2 k}$. This latter problem may be solved without any approximations only numerically. The energy parameters $\kappa$ and the asymptotic coefficients $C_{0}$ as well as their effective-range counterparts $a_{0}$ and $r_{0}$ for $\mathrm{H}^{-}$and the $s$-state negative ions of the alkali metals are presented in Table I [compiled from the data presented in Ref. [37] (for $\mathrm{H}^{-}$) and [40] (for other ions)]. To illustrate the intensity, frequency, and polarization dependences of $\operatorname{Re} \epsilon, \operatorname{Im} \epsilon$, and the coefficients $f_{2 k}$, as well as their sensitivity to the magnitude of the effective range $r_{0}$, we present below numerical results for $\mathrm{H}^{-}$.

\section{A. Intensity, frequency, and polarization dependence of the complex quasienergy of $\mathrm{H}^{-}$: Comparison of TDER and ZRP results}

Since the asymptotic coefficients $C_{\kappa l}$ for real ions differ significantly from those for a ZRP model with the same $\kappa$ (cf. Table I), comparisons of TDER and "pure" ZRP results (i.e., $r_{0}=0, C_{0}^{(Z R P)}=\sqrt{2 \kappa}$ ) show substantial differences. (For the case of circular polarization, such comparisons for the $F$ dependence of the decay rates $\Gamma$ were presented in Ref. [39] for the negative ions $\mathrm{H}^{-}$and $\mathrm{Rb}^{-}$for the third harmonic of the $\mathrm{CO}_{2}$ laser, $\omega=0.351 \mathrm{eV}$.) The comparisons are improved significantly by multiplying the ZRP results for the complex quasienergy by the dimensionless parameter $\mathcal{R}_{0}$ $=\left(C_{0} / C_{0}^{(Z R P)}\right)^{2}$, giving what we henceforth call "renormalized" ZRP (or RZRP) results. As has been shown in Ref. [8], RZRP results for $\mathrm{H}^{-}$over a wide interval of laser intensities are in reasonable agreement with results of other, more sophisticated calculations. We compare below TDER results for the complex quasienergy of $\mathrm{H}^{-}$, obtained by exact solution of Eq. (22), with RZRP results [i.e., exact eigenvalues of Eq. (22) for $r_{0}=0$, multiplied by $\left.\mathcal{R}_{0}\right]$.

The variations of the real and imaginary parts of $\epsilon$ with increasing laser field intensity are presented in Figs. 1 and 2 for two extreme cases of the laser polarization, circular and linear, respectively. Each figure gives results for two wavelengths (long and short) $\lambda=10.6 \mu \mathrm{m} \quad(\omega=0.117 \mathrm{eV}$ $=0.155$ s.u. $)$ and $\lambda=3.5 \mu \mathrm{m}(\omega=0.351 \mathrm{eV}=0.465$ s.u. $)$. For comparison, we present also the lowest-order perturbation theory results for the Stark shift (which depends linearly on the scaled intensity $x=I / I_{0}$ in the perturbative regime) and 

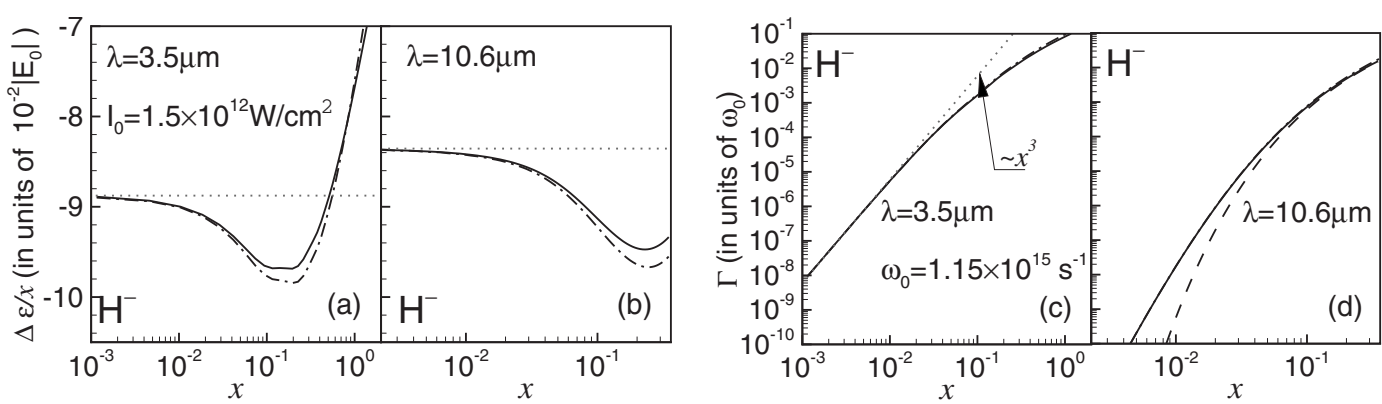

FIG. 1. Dependence of the complex quasienergy of $\mathrm{H}^{-}$on the scaled intensity $x=I / I_{0}$ for circularly polarized fields $(\xi= \pm 1)$ of wavelengths $\lambda=3.5 \mu \mathrm{m}$ and $10.6 \mu \mathrm{m}$. Panels (a) and (b): the Stark shift. Panels (c) and (d): the detachment rate. Solid lines: TDER results. Dot-dashed lines: RZRP results $\left(r_{0}=0\right)$ [i.e., ZRP results multiplied by the factor $\mathcal{R}_{0}$ (cf. Table I)]. Dotted lines: lowest-order perturbation theory results. Dashed line: "static electric field" result [41] (see text discussion). The scaled units of intensity $I_{0}$ and frequency $\omega_{0}$ for the $\mathrm{H}^{-}$ion are given in panels (a) and (c), respectively.

the detachment rates (for $\lambda=3.5 \mu \mathrm{m}$ only). The results for $\Gamma$ at $\lambda=10.6 \mu \mathrm{m}$ correspond to the strong-field regime. As shown in Ref. [41], the decay rate of a weakly bound electron in an $s$ state is insensitive to frequency in the strongfield regime and is well approximated by the cycle averaging of results for an instantaneous static electric field of strength $|\mathbf{F}(t)|$. In agreement with results in Ref. [41], the dashed lines in Figs. 1(d) and 2(d) for $\lambda=10.6 \mu \mathrm{m}$ demonstrate the increasing accuracy of the "static electric field behavior" of $\Gamma$ with increasing intensity.

For the case of circular polarization (Fig. 1) and relatively low intensities, both the Stark shift and the decay rate are well described by the RZRP results. The large value of the renormalization coefficient $\mathcal{R}_{0}$ (cf. Table I) indicates that the unrenormalized ZRP results can only provide qualitative information in this case. For higher intensity, visible discrepancies (up to 20\%) between the TDER and RZRP results occur only for the imaginary part of $\epsilon$, whereas differences for the real part $\operatorname{Re} \epsilon=1+\Delta \epsilon$ are minimal (less than $2 \%$ ). We do not consider intensities for which $|\operatorname{Im} \epsilon|$ becomes larger than $10 \%$ of $|\operatorname{Re} \epsilon|$, since then the concept of a decay rate becomes questionable.

Results for the case of linear polarization (Fig. 2) are qualitatively different from those in Fig. 1. The irregular behavior of $\Gamma$ in Figs. 2(c) and 2(d) is caused by ponderomotive potential-induced closing of the lowest open $n$-photon detachment channels with increasing $F[8,42]$. For $s$ states, such threshold effects are significantly suppressed for the case of circular polarization (cf. Fig. 1) since in this case the threshold behavior of the $n$-photon rate $\Gamma^{(n)}$ is proportional to
$E^{n+1 / 2}$ (according to the Wigner threshold law [43]), where $E=\operatorname{Re} \epsilon+n \omega-u_{p}$. For linear polarization, these channel closing effects are much more pronounced [since $\Gamma^{(n)}$ $\sim E^{1 / 2}\left(E^{3 / 2}\right)$ for even (odd) $\left.n\right]$ and lead also to irregularities in the $F$ dependence of the Stark shift in Figs. 2(a) and 2(b). These irregularities are consequences of the nonanalyticity of the function $\epsilon=\epsilon(F)$ at the "threshold values" $F_{t h}^{(n)}$ of $F$ which are given by the equation $\epsilon\left(F_{t h}^{(n)}\right)+n \omega-\left(F_{t h}^{(n)}\right)^{2} /\left(2 \omega^{2}\right)=0$.

In Fig. 3 we present the frequency dependence of the complex quasienergy for the cases of linear and circular polarizations and $F=0.35$, over a range of frequencies corresponding to $0.43 \leqslant \gamma \leqslant 4.3$, where $\gamma \equiv \omega / F$ is the Keldysh parameter. As for the $F$ dependence, the complex quasienergy results for circular polarization are smooth functions of $\omega$, whereas those for linear polarization reveal distinct threshold peculiarities. (Note that in view of the Stark and ponderomotive shifts, the threshold frequencies are blueshifted compared to the unperturbed values, $\omega_{t h}$ $=1,1 / 2,1 / 3, \ldots .$.

In Fig. 4 we present the dependence of the real and imaginary parts of $\epsilon$ on the degree of circular polarization $|\xi|$ for fixed intensity $(F=0.2)$ and three values of the frequency: $\omega=0.098,0.155$, and 0.235 . For fixed $F$ and $\omega$, both the Stark shift and the detachment rate gradually decrease as the magnitude of $|\xi|$ increases from 0 to 1 . As shown in Fig. 4(a), there is an approximately constant difference between the TDER results for $r_{0} \neq 0$ and the RZRP results for $r_{0}=0$, although it is very small (less than $1 \%$ ), at least for small frequencies. We observe also that the differences between the TDER and RZRP results become smaller as the degree of

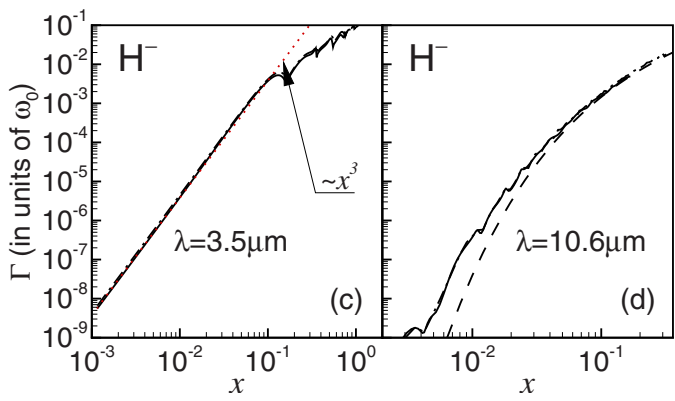

FIG. 2. (Color online) Same as Fig. 1, but for linearly polarized fields. 

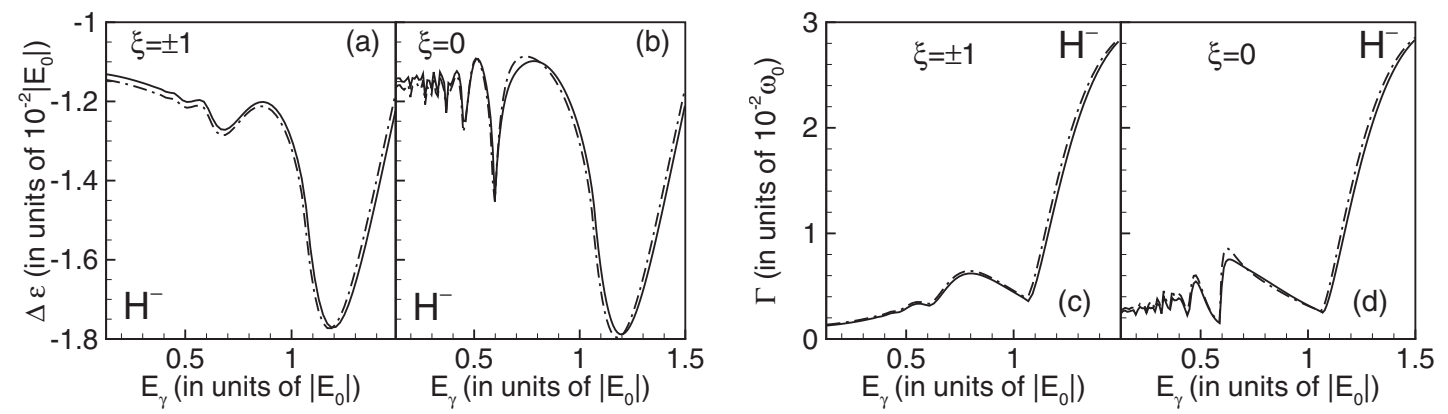

FIG. 3. Variation of the complex quasienergy of $\mathrm{H}^{-}$with photon energy $E_{\gamma}=\hbar \omega$ for a laser field of intensity $I=1.84 \times 10^{11} \mathrm{~W} / \mathrm{cm}^{2}$ and for both circular $(\xi=1)$ and linear $(\xi=0)$ polarizations. Solid lines: TDER results. Dot-dashed lines: RZRP results $\left(r_{0}=0\right)$. For $\omega_{0}$, see Fig. $1(\mathrm{c})$.

circular polarization, $\xi$, increases. This convergence is related in part to the fact that the magnitudes of the shifts and the widths for the $\mathrm{H}^{-}$ion are larger for linear polarization than for circular polarization at the same intensity.

Finally, in Table II we present TDER and RZRP results for detachment rates of $\mathrm{H}^{-}$and the negative alkali-metal ions at three intensities of a linearly polarized $\mathrm{CO}_{2}$ laser field. The data show that the magnitude of $\Gamma$ increases significantly as the binding energy $\left|E_{0}\right|$ decreases (cf. Table I). This trend is understandable since the scaled unit of intensity, $I_{0}$, is proportional to $\left|E_{0}\right|^{3}$. Hence, for fixed intensity $I$, an ion with smaller binding energy $\left|E_{0}\right|$ effectively feels a more intense field (in s.u.) than does an ion with a larger binding energy $\left|E_{0}\right|$. For $\mathrm{H}^{-}$, we compare our results with results of various other calculations at the fundamental $\mathrm{CO}_{2}$ frequency. One sees that our exact TDER results agree with results of recent more detailed calculations [44] within an accuracy better than $8 \%$, with our results being lower in magnitude than those of Ref. [44]. Surprisingly, the RZRP results exhibit almost complete agreement with the results of Ref. [44]. It thus appears that for the case of $\mathrm{H}^{-}$the effective-rangetheory-based renormalization of the ZRP results (cf. Table I) captures the essential corrections necessary to obtain agreement with more accurate single-active-electron model results. We note in addition that the quasiclassical, Keldysh approximation (KA) [45] results of Ref. [46] also agree with the results of Ref. [44] within an accuracy better than $8 \%$, but lie above those of Ref. [44]. (Note, however, that in Ref. [27] it is argued that the $\mathrm{H}^{-}$rates of Ref. [45] should be

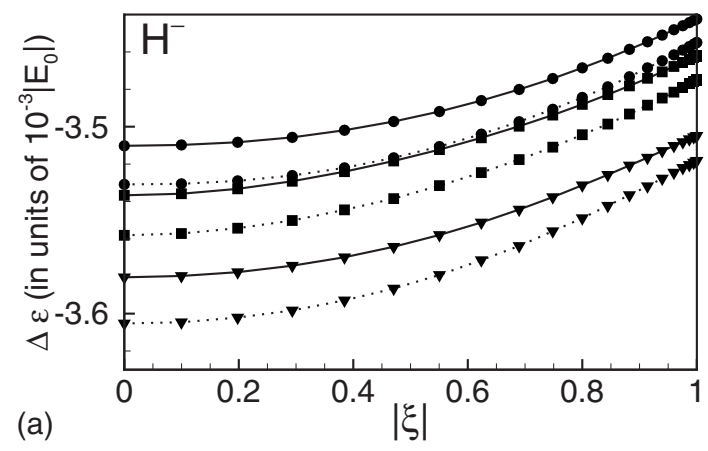

scaled upward by $10.4 \%$.) The tunneling KA results of Ref. [47] lie significantly above the results of all other calculations shown in Table II for these intensities.

For the negative alkali-metal ions at the fundamental frequency of the $\mathrm{CO}_{2}$ laser field, the RZRP and TDER results agree within better than $10 \%$ at all three laser intensities shown. Moreover, in all cases at this frequency the RZRP results are larger than the TDER results. For the third harmonic of the $\mathrm{CO}_{2}$ laser field, however, it is difficult to identify any general trends applicable to all ions. For $\mathrm{H}^{-}$and $\mathrm{Li}^{-}$ the RZRP results are generally larger than the TDER results, in some cases by as much as nearly $20 \%$. This trend, however, begins to reverse in the case of $\mathrm{Na}^{-}$at the lowest intensity shown, where the TDER and RZRP agree essentially exactly. For the cases of $\mathrm{K}^{-}$and $\mathrm{Rb}^{-}$, the RZRP results are a few percent lower than the TDER results at the lowest two intensities and a few percent higher than the TDER results at the highest intensity shown.

\section{B. Plateau features in the fourier coefficients of $f(t)$ : TDER and $Z R P$ results}

An equally important ingredient of the QQES wave function (21) besides the complex quasienergy $\epsilon$ is the periodic function $f(t)$ or, equivalently, the set of its Fourier coefficients $f_{2 k}$, which satisfy the matrix equation (26). Replacing for simplicity the index $2 k$ in $f_{2 k}$ by $k, \Phi_{\epsilon}(\mathbf{r}, t)$ may be represented in terms of the set $f_{k}$ as

$$
\Phi_{\epsilon}(\mathbf{r}, t)=\sum_{k=-\infty}^{\infty} f_{k} e^{-2 i k \omega t} \Phi^{K A}(\mathbf{r}, t),
$$

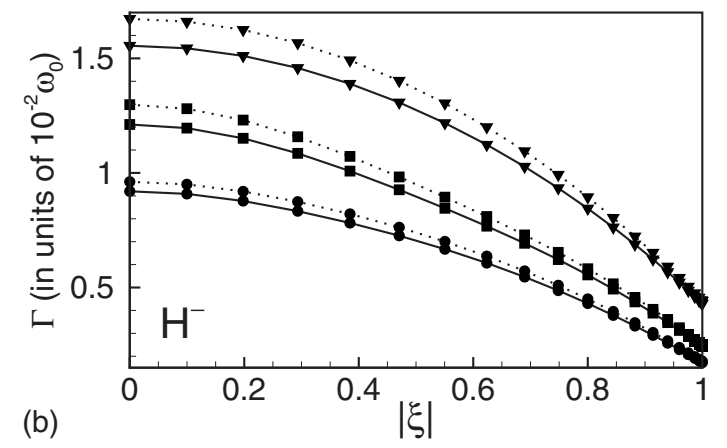

FIG. 4. The $\xi$ dependence (where $\xi$ is the degree of circular polarization) of (a) the Stark shift and (b) the detachment rate for $\mathrm{H}^{-}$for an intensity $I=6 \times 10^{10} \mathrm{~W} / \mathrm{cm}^{2}$ and three values of $\lambda: 16.8 \mu \mathrm{m}$ (circles), $10.6 \mu \mathrm{m}$ (squares), and $7.0 \mu \mathrm{m}$ (triangles). Solid lines: TDER results. Dotted lines: RZRP results. For $\omega_{0}$, see Fig. 1(c). 
TABLE II. Detachment rates (in atomic units) for $\mathrm{H}^{-}$and the negative alkali-metal ions. Results are given for three intensities $I$ (in units of $\left.10^{10} \mathrm{~W} / \mathrm{cm}^{2}\right)$ and two frequencies of a linearly polarized $\mathrm{CO}_{2}$ laser field: the fundamental $(\lambda=10.6 \mu \mathrm{m})$ and the third harmonic $(\lambda$ $=3.5 \mu \mathrm{m}) .(n) \equiv 10^{n}$.

\begin{tabular}{|c|c|c|c|c|}
\hline Ion & Ref. & $I=1$ & $I=3$ & $I=10$ \\
\hline \multicolumn{5}{|c|}{$\lambda=10.6 \mu \mathrm{m}$} \\
\hline \multirow[t]{5}{*}{$\mathrm{H}^{-}$} & TDER & $9.21(-10)$ & $1.80(-7)$ & $1.53(-5)$ \\
\hline & RZRP & $9.47(-10)$ & $1.87(-7)$ & $1.62(-5)$ \\
\hline & {$[44]$} & $9.55(-10)$ & $1.87(-7)$ & $1.65(-5)$ \\
\hline & {$[46]$} & $1.01(-9)$ & $2.02(-7)$ & $1.78(-5)$ \\
\hline & {$[47]$} & $1.23(-9)$ & $2.40(-7)$ & $2.35(-5)$ \\
\hline \multirow[t]{2}{*}{$\mathrm{Li}^{-}$} & TDER & $2.44(-8)$ & $2.03(-6)$ & $5.78(-5)$ \\
\hline & RZRP & $2.51(-8)$ & $2.13(-6)$ & $6.26(-5)$ \\
\hline \multirow[t]{2}{*}{$\mathrm{Na}^{-}$} & TDER & $1.79(-7)$ & $5.59(-6)$ & $1.09(-4)$ \\
\hline & RZRP & $1.86(-7)$ & $5.87(-6)$ & $1.22(-4)$ \\
\hline \multirow[t]{2}{*}{$\mathrm{K}^{-}$} & TDER & $2.52(-7)$ & $8.28(-6)$ & $1.45(-4)$ \\
\hline & RZRP & $2.60(-7)$ & $8.96(-6)$ & $1.56(-4)$ \\
\hline \multirow[t]{2}{*}{$\mathrm{Rb}^{-}$} & TDER & $2.28(-7)$ & $1.30(-5)$ & $1.29(-4)$ \\
\hline & RZRP & $2.32(-7)$ & $1.36(-5)$ & $1.37(-4)$ \\
\hline \multicolumn{5}{|c|}{$\lambda=3.5 \mu \mathrm{m}$} \\
\hline \multirow[t]{2}{*}{$\mathrm{H}^{-}$} & TDER & $3.13(-8)$ & $9.26(-7)$ & $3.69(-5)$ \\
\hline & RZRP & $3.63(-8)$ & $1.04(-6)$ & $3.96(-5)$ \\
\hline \multirow[t]{2}{*}{$\mathrm{Li}^{-}$} & TDER & $2.48(-6)$ & $1.99(-5)$ & $3.78(-5)$ \\
\hline & RZRP & $2.75(-6)$ & $2.27(-5)$ & $4.02(-5)$ \\
\hline \multirow[t]{2}{*}{$\mathrm{Na}^{-}$} & TDER & $3.87(-6)$ & $3.13(-5)$ & $2.32(-4)$ \\
\hline & RZRP & $3.86(-6)$ & $3.24(-5)$ & $2.70(-4)$ \\
\hline \multirow[t]{2}{*}{$\mathrm{K}^{-}$} & TDER & $4.42(-6)$ & $3.55(-5)$ & $2.86(-4)$ \\
\hline & RZRP & $4.21(-6)$ & $3.46(-5)$ & $3.03(-4)$ \\
\hline \multirow[t]{2}{*}{$\mathrm{Rb}^{-}$} & TDER & $3.86(-6)$ & $3.11(-5)$ & $2.53(-4)$ \\
\hline & RZRP & $3.69(-6)$ & $3.02(-5)$ & $2.59(-4)$ \\
\hline
\end{tabular}

$$
\Phi^{K A}(\mathbf{r}, t)=-\sqrt{4 \pi} C_{0} \int_{0}^{\infty} d \tau e^{i(\epsilon+2 k \omega) \tau} G(\mathbf{r}, t ; 0, t-\tau) .
$$

For a ZRP model, a number of general properties of the QQES wave function and the coefficients $f_{k}$ have been discussed in Refs. $[8,15]$. Below we present some additional results for a ZRP model as well as a comparison of the ZRP results with those taking into account the finite value of $r_{0}$ for the $\mathrm{H}^{-}$ion. Moreover, because some general properties of $f_{k}$ (such as their $\omega$ and $F$ dependences and plateau cutoff positions) depend only slightly on the spatial symmetry of the initial state, the results for an $s$ state presented below will be useful for further discussions of the general case of $l>0$.

In Fig. 5 we compare TDER $\left(r_{0} \neq 0\right)$ and ZRP $\left(r_{0}=0\right)$ results for the spectrum of coefficients $f_{k}$ (normalized so that $f_{k=0}=1$ ) for the case of linear polarization. The two results are in fair qualitative agreement. Although both sets of laser parameters correspond to the nonperturbative regime $(\gamma$ $\approx 0.7$ ), the coefficients $f_{k}$ for negative $k$ and for positive $k$ $\leqslant k_{o}$ show a perturbativelike decrease [8]: $f_{k}$ $\approx \alpha_{2 k}(\omega)\left(\ell F^{2}\right)^{|k|}$, where the coefficient $\alpha_{2 k}(\omega)$ is discussed below. The key feature of the $f_{k}$ spectra in Fig. 5 is the existence of a plateau for positive $k$ (starting from $k \approx k_{o}$ ), which is qualitatively similar to the plateau in HHG. For both sets of laser parameters in Fig. 5 (as well as for others) the effects of nonzero $r_{0}$ are most significant in the region of $k$ around the onset of the plateau, indicating that the magnitude of the coefficients $f_{k}$ with $k \approx k_{o}$ is most sensitive to the details of the binding potential. As an analysis of the ZRP model shows [8], the onset position $k_{o}$ is described approximately by

$$
k_{o}=\left[\frac{\left|E_{0}\right|}{2 \omega}\right] \text {, }
$$

where the symbol $[x]$ means the integer part of $x$. This estimate shows that the plateau onset originates from purely quantum effects. Indeed, for a fixed $\omega$, the value $k=k_{o}$ corresponds to the first branch-point nonanalyticity of the parameter $\alpha_{2 k}(\omega)$ as a function of $k$, which stems from the factor $\sqrt{E_{0}+2 k \omega}$. Physically, the plateau onset corresponds to the lowest value of $k$ at which the lowest even $u_{p}$-unshifted photodetachment channel for a given $\omega$ becomes open in the 

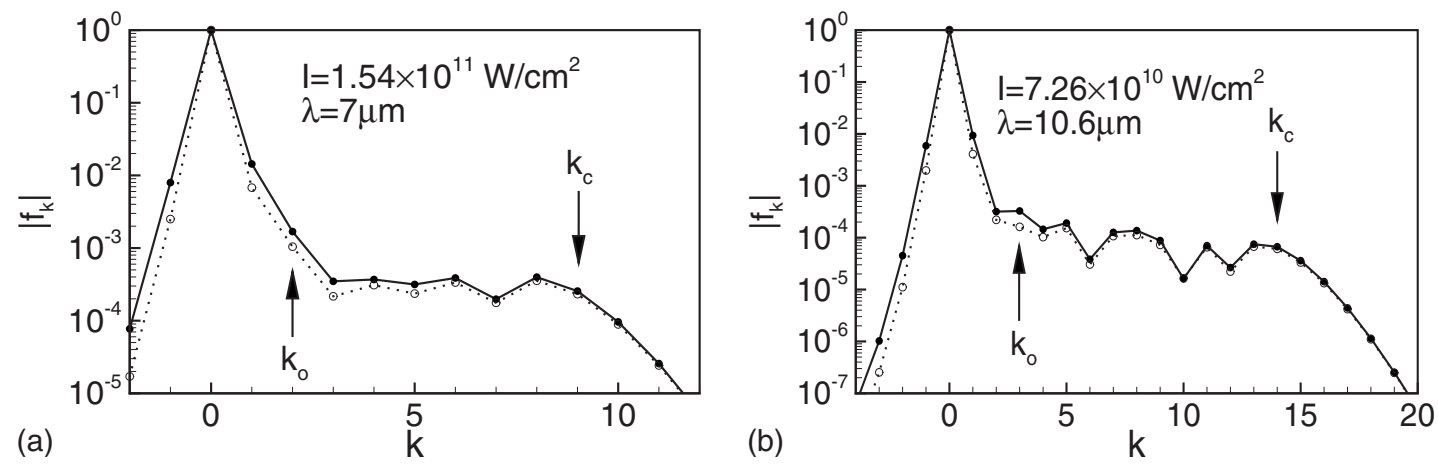

FIG. 5. Comparison of TDER and ZRP results for the spectrum of coefficients $f_{k}$ calculated for two sets of laser intensities and wavelengths. ZRP results $\left(r_{0}=0\right)$ ): dotted lines with open circles. TDER results for the $\mathrm{H}^{-}$ion (using the finite $r_{0}$ value in Table I): solid lines with solid circles. The arrows show the positions of the plateau onset, $k=k_{o}$ [cf. Eq. (32)], and the cutoff, $k=k_{c}$ [cf. Eq. (33)].

process of photon exchange between the electron and laser field at the origin or, equivalently, when the electron energy after exchange of $2 k$ photons (i.e., $E_{0}+2 k \omega$ ) becomes positive. In contrast, although we use a rigorous quantum approach, the cutoff $\left(k=k_{c}\right)$ in the spectrum of $f_{k}$ [8],

$$
k_{c}=\left[\frac{1+3.17 u_{p}}{2 \omega}\right],
$$

is determined by the purely classical quantity $E_{c l}^{(\max )}$ $\approx 3.17 u_{p}$, which is the maximum kinetic energy of a classical electron in a laser field upon returning to the same point $\mathbf{r}$ after some time interval $\Delta t=t-t^{\prime}$ [24].

This remarkable fact demonstrates how classical features appear even in a rigorous quantum treatment of a bound electron in a laser field when the electron-laser interaction becomes stronger than the binding potential. Generally, the appearance of classical features in the strong-field regime may be expected, since the key equation of TDER theory, Eq. (22), contains the classical action of a free electron in a laser field.

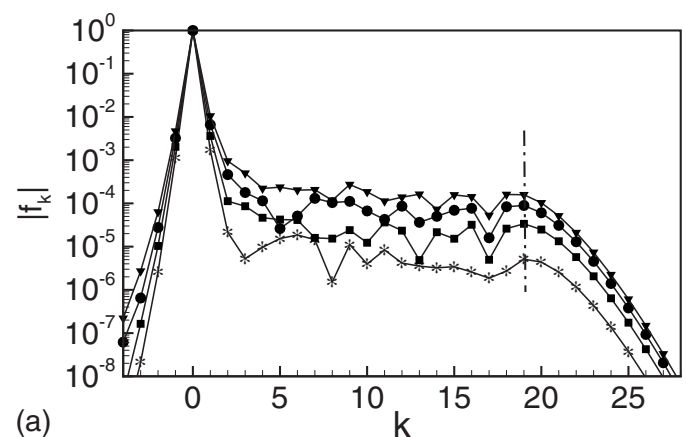

Both estimates (32) and (33) are applicable in the strongfield regime $u_{p} \gg \omega$ and can be obtained from analytical estimates of the matrix elements (B5) similarly to those for a ZRP model [48]. Although the cutoff value $k_{c}$ in the spectrum of $f_{k}$ increases with increasing intensity or decreasing frequency $\left(\sim \omega^{-3}\right)$, the height of the plateau dramatically decreases as $\omega$ decreases (cf. Fig. 6) and tends to zero in the limiting case $\omega \rightarrow 0$. Thus, there exists some optimum between intensity and frequency, for which rescattering effects are most effective (as measured by the magnitude and extent of the plateau).

The dependence of the coefficients $f_{k}$ on the ellipticity of a laser field is shown in Fig. 7. Note that the intensity of rescattering effects is defined by the nondiagonal matrix elements (B5), which decrease in magnitude as the degree of linear polarization, $\ell$, decreases [see the argument of the Bessel function in Eq. (B5)]. For elliptic polarization, not only does the plateau height decrease with decreasing $\ell$, but also both its onset and cutoff positions become $\ell$ dependent; in particular, the $\ell$ dependence of the cutoff position in Fig. 7 is described very well by the formula [48]

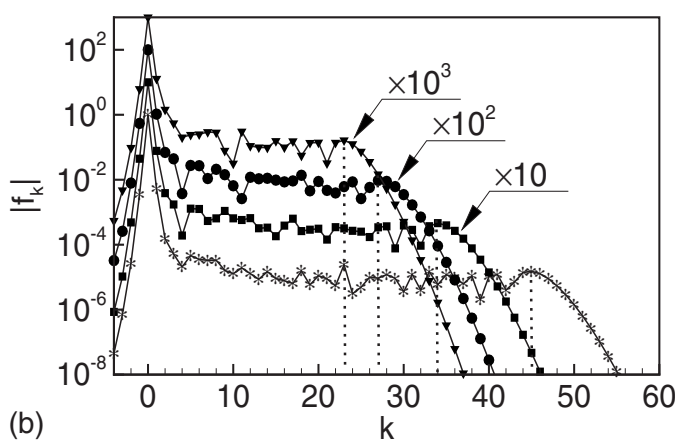

FIG. 6. Spectra of QQES coefficients $f_{k}$ for $\mathrm{H}^{-}$in a linearly polarized field for frequencies and field strengths corresponding to (a) fixed cutoff position and (b) fixed Keldysh parameter. (a) TDER values of $\left|f_{k}\right|$ for different pairs of values of $\omega$ and $F$ (in scaled units) corresponding to a fixed cutoff position (indicated by the vertical dot-dashed line) $k=k_{c}=\left(1+3.17 u_{p}\right) / 2 \omega$ [cf. Eq. (33)]. Stars: $\omega=0.098$ and $F=0.129$. Squares: $\omega=0.128$ and $F=0.200$. Circles: $\omega=0.158$ and $F=0.281$. Triangles: $\omega=0.188$ and $F=0.370$. (b) TDER values of $\left|f_{k}\right|$ for different pairs of values of $\omega$ and $F$ (in scaled units) corresponding to a fixed Keldysh parameter $\gamma=\omega / F=0.45$. For better visualization, each curve is multiplied by the factor $10^{\mu}$. Stars: $\omega=0.098, F=0.218$, and $\mu=0$. Squares: $\omega=0.128, F=0.284$, and $\mu=1$. Circles: $\omega=0.158, F$ $=0.351$, and $\mu=2$. Triangles: $\omega=0.188, F=0.418$, and $\mu=3$. Vertical dotted lines mark the cutoff positions $k=k_{c}$ according to Eq. (33). Solid lines in (a) and (b) serve to guide the eye. 


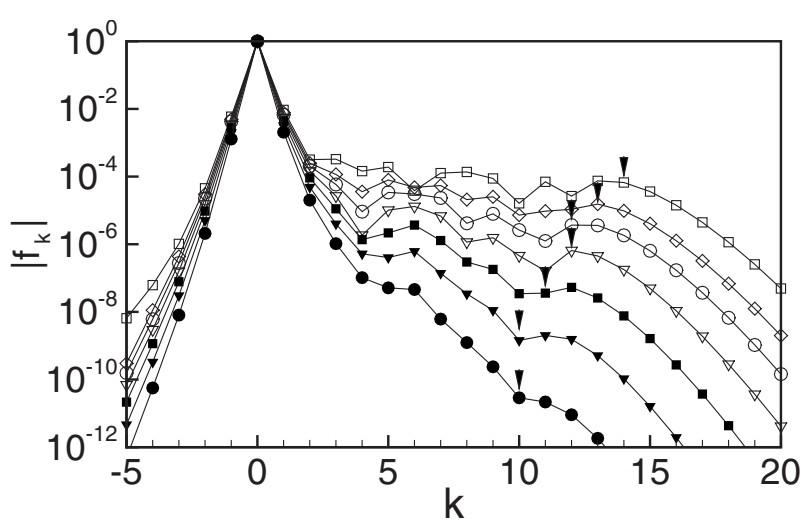

FIG. 7. Spectrum of coefficients $f_{k}$ for $\mathrm{H}^{-}$in an elliptically polarized field with $F=7.3 \times 10^{10} \mathrm{~W} / \mathrm{cm}^{2}, \lambda=10.6 \mu \mathrm{m}$, and several values of $\ell$. Open squares: $\ell=1$. Open diamonds: $\ell=0.835$. Open circles: $\ell=0.724$. Open triangles: $\ell=0.6$. Solid squares: $\ell=0.471$. Solid triangles: $\ell=0.342$. Solid circles: $\ell=0.22$. Arrows mark the cutoff positions $k=k_{c}$ according to Eq. (33).

$$
k_{c}=\left[\frac{1+3.17 u_{p}(1+\ell) / 2}{2 \omega}\right],
$$

where $u_{p}(1+\ell) / 2$ may be considered as the "longitudinal" ponderomotive shift corresponding to the component of the electric field $\mathbf{F}(t)$ in Eq. (3) along the major axis of the polarization ellipse. Note that Eq. (34) is an asymptotic estimate, valid for $u_{p} \ell \gg \omega$, so that some inaccuracy of order \pm 1 for $k_{c}$ is expected for small $\ell$. For relatively small $\ell$, there is no well-defined plateau feature in the spectrum of $f_{k}$, so that $k_{c}$ in these cases is indicative only of where the cutoff would be if there were a plateau (see, e.g., the curves for $\ell=0.471$, 0.342 , and 0.22 in Fig. 7).

\section{CASE OF AN INITIAL $p$ STATE IN A LINEARLY POLARIZED FIELD}

\section{A. General considerations and QQES wave functions for $p$ states}

Since the explicit form of $\Phi_{\epsilon}(\mathbf{r}, t)$ outside the well $U(r)$ depends significantly on the angular momentum value $l$ of the initial state and on the symmetry of the dipole interaction operator $V(\mathbf{r}, t)$ (i.e., on the polarization state of the laser field), general results for arbitrary $l$ and arbitrary laser polarization are rather complicated. Thus, for bound states with nonzero angular momentum, we begin with results for linear polarization. For this case, the Hamiltonian in Eq. (7) possesses axial symmetry in the reference frame with the $z$ axis along the laser polarization, so that the angular momentum projection $m$ on this axis is a conserved quantum number. Thus the set of $2 l+1$ QQESs corresponding to the degenerate initial state $\psi_{\kappa l m}(\mathbf{r})$ may be labeled by the index $m$. Since $m$ is the only pseudoscalar quantity of the problem, the complex quasienergies of these QQESs depend only on $\mathrm{m}^{2}$, so that the QQESs in a linearly polarized field, $\Phi_{\epsilon_{\mid m}, m}(\mathbf{r}, t)$, are doubly degenerate with respect to the sign of $m$. Thus the boundary condition at small $r$ is formulated for different $m$ separately and has the form (12). For given $l$ and $m$, integro- differential equations for the functions $f^{(m)}(t)$ and corresponding quasienergies $\epsilon_{|m|}$ follow by matching this boundary condition and the small-r expansion of the QQES wave function in Eq. (18). In general, the Stark shift and splitting of an initial energy $E_{0}, \Delta \epsilon_{|m|}=\operatorname{Re} \epsilon_{|m|}-E_{0}$, may be parametrized as

$$
\Delta \epsilon_{|m|}=-\frac{1}{4}\left[\alpha(\omega, F)+\beta(\omega, F) m^{2}\right] F^{2},
$$

where the parameters $\alpha(\omega, F=0)$ and $\beta(\omega, F=0)$ do not depend on $m^{2}$ and may be expressed in terms of standard scalar and tensor dynamic polarizabilities (see, e.g., [10]). An important property of $\Phi_{\epsilon_{|m|}, m}(\mathbf{r}, t)$ is that its expansion in spherical harmonics $Y_{l^{\prime}, m^{\prime}}(\hat{\mathbf{r}})$ involves only harmonics with $m^{\prime}$ $=m$ and $l^{\prime} \geqslant|m|$, so that for states with $m \neq 0$ the active electron, even in virtual intermediate states, always has nonzero angular momentum. Thus it may be expected that the influence of a strong field on states with $m \neq 0$ should be suppressed compared to the case of $m=0$ since (i) the centrifugal barrier significantly suppresses rescattering effects in the former case and (ii) the electron density in a state with $m$ $=0$ is mostly localized along the electric vector $\mathbf{F}(t)$. These considerations help to interpret our quantitative results for the parameters of the QQESs for a degenerate initial bound state of $p$ symmetry [such as the Stark shift $\Delta \epsilon_{|m|}$, the detachment rate $\Gamma_{|m|}=-2 \operatorname{Im} \epsilon_{|m|}$, and the Fourier coefficients $\left.f^{(m)}(t)\right]$.

Taking into account Eqs. (A4) and (A5), Eq. (18) gives the following result for the QQES wave function of a $p$ state outside the potential well:

$$
\begin{aligned}
\Phi_{\epsilon_{|m|}, m}(\mathbf{r}, t)= & 2 \pi i \int_{0}^{\infty} e^{i \epsilon_{|m|} \tau} G(\mathbf{r}, t ; 0, t-\tau) \\
& \times\left[\frac{r}{\tau} Y_{1, m}(\hat{\mathbf{r}})+\delta_{m, 0} \sqrt{\frac{3}{4 \pi}} \varphi(t, \tau)\right] f^{(m)}(t-\tau) d \tau,
\end{aligned}
$$

where

$$
\varphi(t, \tau)=\frac{2 F}{\omega}\left[\frac{2 \sin (\omega \tau / 2) \sin \omega(t-\tau / 2)}{\omega \tau}-\sin \omega(t-\tau)\right],
$$

and $f^{(m)}(t)=\sum_{k} f_{k}^{(m)} e^{-2 i k \omega t}$. The integro-differential equation that follows from matching the small- $r$ expansion of the wave function (36) [cf. Eq. (A12) for $\ell=1$ ] to the boundary condition (12) is equivalent to the following infinite system of linear equations for the coefficients $f_{k}^{(m)}$ and the quasienergy $\epsilon$ :

$$
\mathcal{R}(E) f_{k}^{(m)}=\sum_{k^{\prime}}\left[M_{k, k^{\prime}}(\epsilon)+\delta_{m, 0} \tilde{M}_{k, k^{\prime}}(\epsilon)\right] f_{k^{\prime}}^{(m)},
$$

where $E=\epsilon+2 k \omega$,

$$
\mathcal{R}(E)=-a_{1}^{-1}+r_{1} E / 2-i E^{3 / 2},
$$

and explicit forms for the matrix elements $M_{k, k^{\prime}}(\epsilon)$ and $\tilde{M}_{k, k^{\prime}}(\epsilon)$ are given in Appendix B. 
TABLE III. Parameters for negative ions having valence $p$ electrons.

\begin{tabular}{lcccccc}
\hline \hline Ion & $\left|E_{0}\right|(\mathrm{eV})$ & $\kappa($ a.u. $)$ & $C_{\kappa 1}($ a.u $)$ & $C_{\kappa 1}($ s.u. $)$ & $a_{1} \kappa^{3}$ & $r_{1} \kappa^{-1}$ \\
\hline $\mathrm{O}^{-}$ & 1.464 & 0.328 & 0.65 & 1.135 & 0.784 & -4.553 \\
$\mathrm{~F}^{-}$ & 3.401 & 0.500 & 0.84 & 1.188 & 0.827 & -4.417 \\
$\mathrm{Cl}^{-}$ & 3.622 & 0.516 & 1.34 & 1.865 & 1.270 & -3.575 \\
$\mathrm{Br}^{-}$ & 3.374 & 0.498 & 1.49 & 2.111 & 1.381 & -3.449 \\
$\mathrm{I}^{-}$ & 3.070 & 0.475 & 1.90 & 2.757 & 1.583 & -3.263 \\
\hline \hline
\end{tabular}

\section{B. Numerical results for halogen anions}

In Table III we present data for a number of negative ions with valence $p$-state electrons (from Ref. [40]). The variations in the magnitudes of the scattering lengths $a_{1}$ and the effective ranges $r_{1}$ are much larger than for ions having valence $s$-state electrons (cf. Table I). In particular, the asymptotic coefficients $C_{\kappa 1}$ differ by a factor of 3 from $\mathrm{O}^{-}$ (where $C_{\kappa 1}=0.65$ a.u.) to $\mathrm{I}^{-}$(where $C_{\kappa 1}=1.90$ a.u.). Thus it may be expected that the numerical results should be more sensitive to the actual values for $r_{1}$ in the equations for $\epsilon_{\mid m}$ and $f_{k}^{(m)}$. In earlier works $[46,47,49]$ employing the KA [45] for states with nonzero angular momentum, different ions are distinguished through both the normalization factors $C_{\kappa l}$ and the binding energy $\left|E_{0}\right|$, as in TDER theory. However, for a given $l$, the detachment rate $\Gamma$ in the KA approach factorizes into the product of $C_{\kappa l}^{2}$ and some universal function of $\left|E_{0}\right|$, $F$, and $\omega$. In the TDER theory this latter function depends also on $C_{\kappa l}$ or, equivalently, $r_{l}$. The TDER theory description thus allows one to verify the accuracy of such KA-like approximations.

To illustrate qualitative features of the intensity dependence of $\Delta \epsilon_{|m|}$ and $\Gamma_{|m|}$ for a $p$ state, we choose the $\mathrm{F}^{-}$ion, for which recent experiments [13,50-52] have been performed. In Fig. 8 we present the $F$ dependence of $\Delta \epsilon_{|m|}$ and $\Gamma_{|m|}$ for laser frequencies $\lambda=1.8 \mu \mathrm{m}$ [13] and $\lambda=800 \mathrm{~nm}$ $[50]$. The results show that virtual electron transitions to intermediate $s$ states, which are forbidden for the $|m|=1$ states, significantly increase the absolute values of both the Stark shift and the detachment rate for the state with $m=0$. One sees also that deviations from the perturbation theory (PT) results for $\Delta \epsilon_{|m|}$ are small up to much stronger fields than in

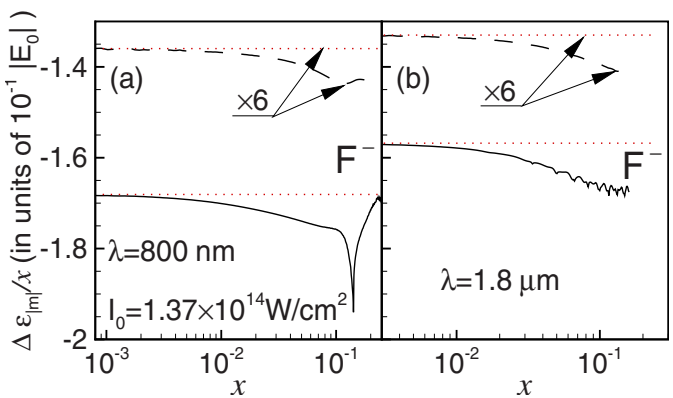

the case of the detachment rates, because $\Delta \epsilon_{|m|}$ is linear in the laser intensity in the PT regime, whereas $\Gamma_{|m|}$ is of much higher order in $I$ in this regime: $\Gamma_{|m|} \sim I^{n_{0}}$, where $n_{0}$ $=\left[\left|E_{0}\right| /(\hbar \omega)+1\right]$. Irregularities in the dependence of $\Gamma_{m=0}$ on $F$ are caused by the closing of the lowest open detachment channels due to increases of both the ponderomotive energy and the Stark shift with increasing intensity. This explains why at small frequencies these irregularities become visible in the curve for $\Delta \epsilon_{m=0}(F)$ at $\lambda=1.8 \mu \mathrm{m}$ in Fig. 8(b) only at high intensities, when nonlinear in the intensity corrections to $\Delta \epsilon_{m=0}(F)$ become significant. For higher frequencies (when $n_{0}$ becomes smaller), the channel closing effects do become significant for $\Delta \epsilon_{m=0}(F)$ [cf. Fig. 8(a)]. The fact that threshold effects are significant only for states with $m=0$ and are suppressed for those with $|m|>0$ is clear from the dependence of the Wigner threshold law for the detachment cross section $\sigma$ on the angular momentum $L$ of the detached electron [43]: $\sigma \sim E^{L+1 / 2}$. For instance, for $m=0$ both $\Delta \epsilon_{|m|}$ and $\Gamma_{|m|}$ in Fig. 8 are strongly affected at $\lambda=800 \mathrm{~nm}$ and $F$ $\approx 0.376$ (which corresponds to the closing of the threephoton detachment channel). However, for $|m|=1$ they are smooth. This is so because for $m=0$ the $L=0$ channel gives the dominant contribution to $\Gamma_{|m|}$, whereas for $|m|=1$ only the $L=2$ channel contributes.

The breakdown of PT for $\Gamma_{|m|}$ is illustrated for $\lambda$ $=800 \mathrm{~nm}$ in Fig. 8(c), where deviations from the lowestorder PT behavior, $\Gamma_{|m|} \sim F^{2 n_{0}}$ with $n_{0}=3$, become important for $F \gtrsim 0.2$. Account of high-order PT terms (not shown) improves the results only in a narrow interval of $F(F$ $<0.35$ ) below the closing of the three-photon detachment channel, where PT for the complex quasienergy becomes divergent [53]. In contrast to the results for $\lambda=800 \mathrm{~nm}$, the

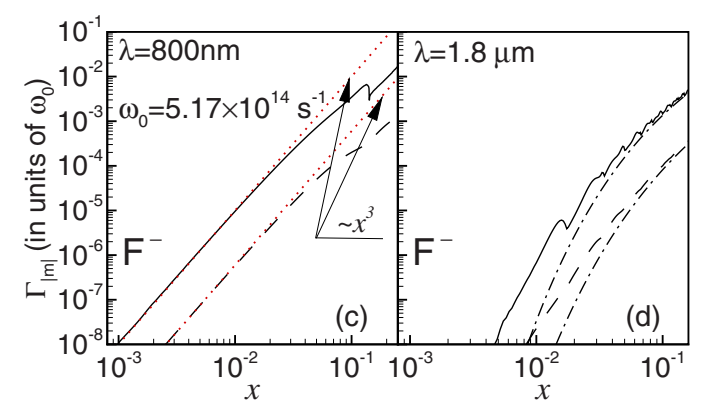

FIG. 8. (Color online) Dependence of the complex quasienergy of $\mathrm{F}^{-}$on the reduced intensity $x=I / I_{0}$ for linearly polarized fields of wavelengths $\lambda=800 \mathrm{~nm}$ and $1.8 \mu \mathrm{m}$. Panels (a) and (b): the Stark shift. Panels (c) and (d): the detachment rate. Solid lines: TDER results for $m=0$. Dashed lines: TDER results for $|m|=1$. Dotted lines: lowest-order perturbation theory results. Dash-dotted lines: "static electric field" results (cf. Fig. 1). Results in panels (a) and (b) for $|m|=1$ are multiplied by a factor of 6 for better visualization. Numerical values for $I_{0}$ and $\omega_{0}$ are presented in the figures. 

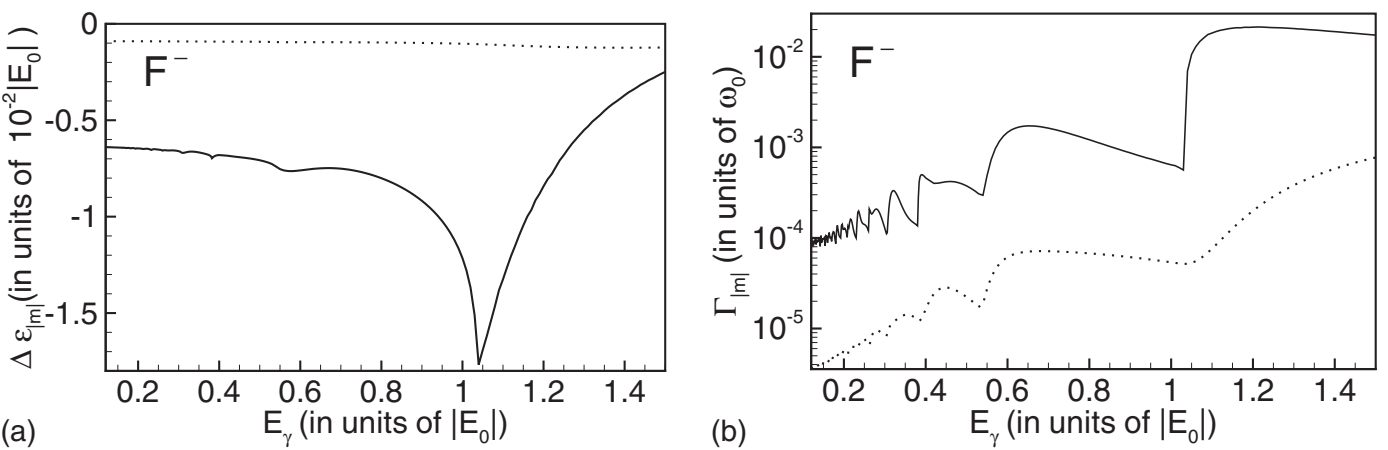

FIG. 9. Complex quasienergy of $\mathrm{F}^{-}$vs photon energy $E_{\gamma}=\hbar \omega$ for a linearly polarized field of intensity $I=5.48 \times 10^{12} \mathrm{~W} / \mathrm{cm}^{2}$. Solid lines: TDER results for $m=0$. Dotted lines: TDER results for $|m|=1$. For $\omega_{0}$, see Fig. 8.

results for $\Gamma_{|m|}$ at $\lambda=1.8 \mu \mathrm{m}$ in Fig. 8(d) correspond to the deeply nonperturbative regime, since the five-photon detachment channel is already closed for the intensities considered there and the first zigzag on the curve for $\Gamma_{m=0}$ corresponds to the closing of the six-photon channel. For such intensities, the "static electric field" results (cf. Figs. 1 and 2), given by the dash-dotted lines in Fig. 8(d) for $\lambda=1.8 \mu \mathrm{m}$, confirm the applicability of the method developed in Ref. [41] for $s$ states to the case of $p$ states with $m=0, \pm 1$.

Irregularities in the frequency dependence of $\Delta \epsilon_{|m|}$ and $\Gamma_{|m|}$, shown in Fig. 9, are also caused by the threshold effects discussed above. They correspond, however, to the opening of detachment channels requiring a smaller number of absorbed photons, $n_{0}$, as $\omega$ increases. The most pronounced "channel opening effects" occur in the vicinity of the threshold for one-photon detachment, which is blueshifted due to both ponderomotive and Stark shifts. Across this threshold one observes the downward cusp shape of the curve for $\Delta \epsilon_{m=0}$ and the sharp step in that for $\Gamma_{m=0}$, both of which stem primarily from detachment of the initially bound, $p$-state electron into the $s$-wave continuum channel. In contrast, one observes a much smoother step across this threshold in the curve for $\Gamma_{|m|=1}(\omega)$, which originates entirely from photodetachment of the initially bound $p$ electron into the $d$-wave channel. Near the threshold for two-photon detachment (at $\omega \approx 0.52)$, the behaviors of both $\Gamma_{m=0}(\omega)$ and $\Gamma_{|m|=1}(\omega)$ are essentially identical, since both are governed by the Wigner law for $L=1$.

The spectra of Fourier coefficients $f_{k}^{(m)}$ of the functions $f^{(m)}(t)$ in Eq. (36) are shown in Fig. 10 for $\mathrm{I}^{-}$and $\mathrm{F}^{-}$ions together with results for the bound $s$ state in a ZRP model for the same scaled laser parameters. One sees that the $p$-state results are very similar to those for valence $s$-state ions (cf. Sec. III B) except for those substates having $|m|=1$, for which the plateau height (or the "intensity of rescattering") is significantly suppressed owing to the fact that the electron angular momentum cannot be zero in this case (cf. Sec. IV A). The absolute values of the coefficients $f_{k}^{(m)}$ for different ions differ considerably for negative $k$ and for positive $k$ prior to the onset of the plateau. In contrast, both the ZRP results and the results for $m=0$ over the region of the plateau (and beyond) are essentially independent of the kind of ion. Therefore, rescattering effects are largely insensitive to the absolute value of the angular momentum of an initially bound electron, but depend crucially on the population of the $s$-wave component of the laser-dressed QQES wave function.

In Table IV we present our TDER results for the detachment rates of a number of ions and compare them with tunnelling KA rates [47] and results of the more precise KA of Ref. [46], in which the authors do not use the lowmomentum expansions of the $n$-photon detachment rates. In a way similar to that for a ZRP model [23], in the TDER theory the KA result for the complex quasienergy follows from the exact TDER equations (38) for $\epsilon$ and $f_{k}^{(m)}$ upon neglecting all Fourier coefficients $f_{k}^{(m)}$ except $f_{0}^{(m)}=1$ and, furthermore, approximating $\epsilon=E_{0}=-1$ in the matrix elements $M_{0,0}(\epsilon)$ and $\tilde{M}_{0,0}(\epsilon)$. Then, provided that $\left|\Delta \epsilon_{|m|} / E_{0}\right|$ $\ll 1$, the Stark shift and detachment rate may be calculated as the real and imaginary parts of $\Delta \epsilon_{|m|}$, which (using the relation, $\left.r_{1}+3=-2 C_{\kappa 1}^{-2}\right)$ is given by

$$
\Delta \epsilon_{|m|}=-C_{\kappa 1}^{2}\left[M_{0,0}\left(E_{0}\right)+\delta_{m, 0} \tilde{M}_{0,0}\left(E_{0}\right)\right] .
$$

(Analytical expressions for KA amplitudes of $n$-photon detachment from $p$ states in the TDER theory can be found in Refs. $[11,12]$.) For the laser parameters used in Table IV, our numerical KA results for $\Gamma_{|m|}$, obtained according to Eq. (40), are close to those of Ref. [46]. As the data in Table IV indicate (and our more extensive numerical analysis shows), the

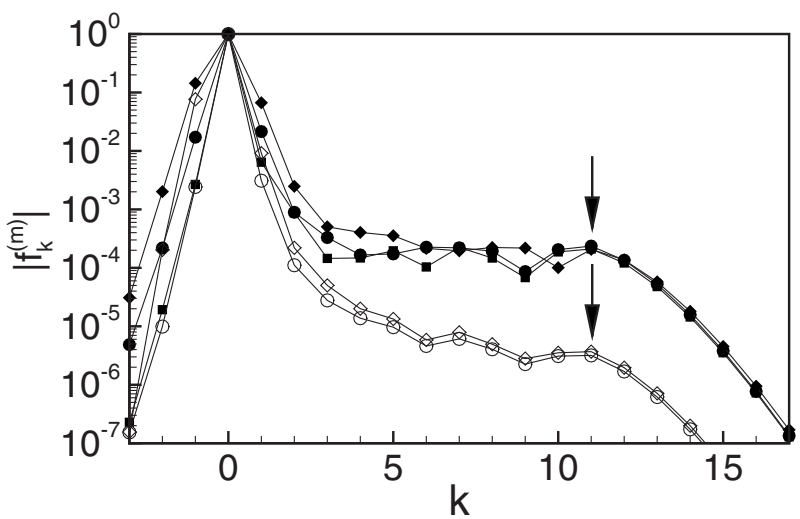

FIG. 10. Spectra of the Fourier coefficients $f_{k}^{(m)}$ for $\mathrm{I}^{-}$(diamonds), $\mathrm{F}^{-}$(circles), and a ZRP model (squares) for the case of linear polarization, $F=0.3$, and $\omega=0.203$ (in scaled units). Solid and open symbols correspond to $m=0$ and $|m|=1$. Arrows mark the cutoff position according to Eq. (33). Lines are added for better visualization. 
TABLE IV. Rates $\Gamma_{|m|}$ (in a.u.) for detachment of three negative ions by linearly polarized light of intensity $10^{13} \mathrm{~W} / \mathrm{cm}^{2}$ and two wavelengths $\lambda=1.8 \mu \mathrm{m}$ and $800 \mathrm{~nm}$. $(n) \equiv 10^{n}$.

\begin{tabular}{|c|c|c|c|c|c|c|}
\hline \multirow[b]{2}{*}{ Ref. } & \multicolumn{2}{|c|}{$\mathrm{F}^{-}$} & \multicolumn{2}{|c|}{$\mathrm{Cl}^{-}$} & \multicolumn{2}{|c|}{$\mathrm{Br}^{-}$} \\
\hline & $m=0$ & $|m|=1$ & $m=0$ & $|m|=1$ & $m=0$ & $|m|=1$ \\
\hline & & & $\lambda=1.8 \mu \mathrm{m}$ & & & \\
\hline TDER & $9.40(-5)$ & $5.08(-6)$ & $1.17(-4)$ & $6.94(-6)$ & $2.68(-4)$ & $1.65(-5)$ \\
\hline KA & $1.09(-4)$ & $5.25(-6)$ & $1.72(-4)$ & $7.39(-6)$ & $3.58(-4)$ & $1.77(-5)$ \\
\hline [46] & $1.02(-4)$ & $5.65(-6)$ & $1.60(-4)$ & $7.59(-6)$ & $3.58(-4)$ & $2.05(-5)$ \\
\hline \multirow[t]{2}{*}[47]{} & $1.20(-4)$ & $9.09(-6)$ & $1.90(-4)$ & $1.18(-5)$ & $4.30(-4)$ & $3.31(-5)$ \\
\hline & & & $\lambda=800 \mathrm{~nm}$ & & & \\
\hline TDER & $2.12(-4)$ & $1.44(-5)$ & $4.04(-4)$ & $2.03(-5)$ & $6.05(-4)$ & $4.59(-5)$ \\
\hline KA & $2.46(-4)$ & $1.54(-5)$ & $4.26(-4)$ & $2.10(-5)$ & $8.14(-4)$ & $5.15(-5)$ \\
\hline$[46]$ & $2.26(-4)$ & $1.47(-5)$ & $3.92(-4)$ & $2.24(-5)$ & $8.04(-4)$ & $5.26(-5)$ \\
\hline [47] & $3.00(-4)$ & $2.54(-5)$ & $5.30(-4)$ & $3.56(-5)$ & $1.05(-3)$ & $9.16(-5)$ \\
\hline
\end{tabular}

range of validity of the results of Ref. [47] is limited to low frequencies $(\omega<0.2)$ and not too intense fields $(F<0.2)$, whereas our KA results and those of Ref. [46] are applicable over a wider interval of both frequency and intensity. As one sees from the results in Table IV, all approximations overestimate the exact TDER results, owing to their neglect of all but one of the coefficients $f_{k}^{(m)}$. However, the magnitude of this inaccuracy is not so large for the laser parameters shown, although it increases with increasing laser frequency and intensity. This small contribution of the Fourier coefficients $f_{k}^{(m)}$ to the total detachment rates is not surprising, since they decrease in magnitude quickly with increasing $|k|$ (e.g., $\left.f_{|k|=1}^{(m)} \lesssim 0.1 f_{0}^{(m)}\right)$. However, they play a crucial role in describing $n$-photon detachment rates on the high-energy plateaus in both ATD and HHG spectra $[11,17]$.

\section{CASE OF AN INITIAL $p$ STATE IN A CIRCULARLY POLARIZED FIELD}

\section{A. General considerations for circular polarization}

As discussed in Sec. II and illustrated in Sec. III for the case of an $s$ state, for a nondegenerate initial state $\psi_{0}(\mathbf{r})$ subjected to a circularly polarized field all quasienergy harmonics $\Phi_{k \neq 0}(\mathbf{r})$ in Eq. (6) vanish at small distances $r \ll \kappa^{-1}$. Assuming that the quantization axis is chosen along the photon wave vector $\mathrm{k}$, this fact is a consequence of the selection rule $\Delta m=2 \xi$ (or $-2 \xi$ ) for absorption (or emission) of two right-hand $(\xi=+1)$ or left-hand $(\xi=-1)$ polarized photons. For initial states with $l>0$ that are degenerate in $m$, this selection rule is consistent with the selection rule $\Delta l=0$ for emission or absorption of an even number of photons. Thus an electron in the initial state $\psi_{0}(\mathbf{r})=\psi_{\kappa l m}(\mathbf{r})$ can absorb $(k$ $>0)$ or emit $(k<0)$ an even number of circularly polarized photons, $2|k|$, keeping the angular momentum $l$ and populating quasienergy harmonics with the same $l$ but $m^{\prime}=m+2 \xi k$. (Obviously, only $2 l$ such harmonics with $1 \leqslant|k| \leqslant l$ are populated.) Moreover, after turning on the circularly polarized field, the initially degenerate substates $\psi_{\kappa l m}(\mathbf{r})$ with different $m$ evolve (without mixing in $m$ ) into $(2 l+1)$ QQESs having different quasienergies $\epsilon^{(1)}, \epsilon^{(2)}, \ldots, \epsilon^{(2 l+1)}$. Indeed, since besides $m$ the problem involves another pseudoscalar quantity $\xi$ [28], the quasienergy in a circularly polarized field depends on the sign of $m$ through the scalar combination $\xi m$. Thus, for the case of circular polarization, the parametrization (35) for the Stark shift and splitting is modified:

$$
\Delta \epsilon_{m}=-\frac{1}{4}\left[\alpha(\omega, F)+\beta(\omega, F) m^{2}+\xi \gamma(\omega, F) m\right] F^{2} .
$$

In general, the scalar parameter $\gamma(\omega, F)$ depends on $m^{2}$ and, at $F=0$, reduces to the axial (or vector) dynamic polarizability, which vanishes as $\omega \rightarrow 0$ (see, e.g., Ref. [10]). Since for $F \rightarrow 0$ the wave function of each of the $(2 l+1)$ Stark-split QQESs reduces to an unperturbed state $\psi_{\kappa l m}(\mathbf{r})$ with a definite projection $m$, we will continue to use the indices $l$ and $m$ for enumeration of these QQESs, $\Phi_{\epsilon}(\mathbf{r}, t) \equiv \Phi_{l, m}(\mathbf{r}, t)$, and the corresponding complex quasienergies, $\epsilon_{m}$. The general form of the Fourier harmonics $\Phi_{k}(\mathbf{r})$ is clear from the general structure of the Fourier expansion for $\Phi_{l, m}(\mathbf{r}, t)$, which follows from Eq. (18) for $\xi= \pm 1(\ell=0)$ :

$$
\Phi_{l, m}(\mathbf{r}, t)=\sum_{k} e^{i(m+\xi k) \varphi} \phi_{k}(r, \sin \theta) e^{-i k \omega t},
$$

where the $k$ th quasienergy harmonic $\Phi_{k}(\mathbf{r})$ has been expressed as $\Phi_{k}(\mathbf{r})=\phi_{k}(r, \sin \theta) \exp [i(m+\xi k) \varphi]$, where $\varphi$ and $\theta$ are the spherical angles of the vector $\mathbf{r}$. We emphasize that $\Phi_{l, m}(\mathbf{r}, t)$ is not an eigenfunction of the operator $\hat{L}_{z}$. The only symmetry of the QQES wave function in a circularly polarized field is that its $k$ th quasienergy harmonic $\Phi_{k}(\mathbf{r})$ corresponds to a fixed magnetic quantum number $m+\xi k$ so that the partial-wave expansion of this harmonic involves only spherical harmonics $Y_{l^{\prime}, m+\xi k}(\hat{\mathbf{r}})$ having $l^{\prime} \geqslant|m+\xi k|$.

Based on these symmetry considerations, the boundary condition at small $r$ for the $l$-wave component of the QQES wave function in a circularly polarized field (having, for definiteness, $\xi=+1)$ is [cf. Eq. (12)] 


$$
\begin{aligned}
\Phi_{l, m}(\mathbf{r}, t) \sim & \sum_{m^{\prime}} Y_{l, m+m^{\prime}}(\hat{\mathbf{r}}) \\
& \times\left[\frac{1}{r^{l+1}}+\cdots+B\left(\epsilon+m^{\prime} \omega\right)\left(r^{l}+\cdots\right)\right] f_{m^{\prime}}^{(m)} e^{-i m^{\prime} \omega t},
\end{aligned}
$$

where the prime on the summation in Eq. (43) means that only even values of $m^{\prime}$ are summed-i.e., $m^{\prime}=0, \pm 2, \ldots$. Thus the equation for $\epsilon_{m}$ splits into two independent systems involving either even or odd values of $m$. We note also that although Eq. (43) involves nonzero Fourier coefficients, $f_{m^{\prime}}^{(m)}$ of $\Phi_{l, m}(\mathbf{r}, t)$ at small $r$, these coefficients have another, "geometrical" origin compared to the "dynamical" coefficients $f_{n}^{(m)}$ in Eq. (12) for the case of linear polarization, in which the quantum number $m$ is conserved and the index $n$ runs over the infinite interval $n=0, \pm 2, \pm 4, \ldots$.

In accordance with the boundary condition (43) and the general form (18) of the QQES having a definite value of the projection $m$, the proper QQES solution outside the well $U(r)$ for the case of circular polarization may be expressed as a superposition of derivatives of the Green's function:

$$
\begin{aligned}
\Phi_{l, m}(\mathbf{r}, t)= & -4 \pi C_{\kappa l} \sum_{m^{\prime}}^{\prime} f_{m^{\prime}}^{(m)} e^{-i m^{\prime} \omega t} \int_{-\infty}^{t} d t^{\prime} e^{i\left(\epsilon_{m}+m^{\prime} \omega\right)\left(t-t^{\prime}\right)} \\
& \times\left.\mathcal{Y}_{l, m+m^{\prime}}\left(\nabla_{\mathbf{r}^{\prime}}\right) G\left(\mathbf{r}, t ; \mathbf{r}^{\prime}, t^{\prime}\right)\right|_{\mathbf{r}^{\prime}=0}
\end{aligned}
$$

Taking into account Eqs. (16)-(18), one obtains the following final result:

$$
\begin{aligned}
\Phi_{l, m}(\mathbf{r}, t)= & -4 \pi(-i)^{l} C_{\kappa l} \\
& \times \sum_{m^{\prime}}^{\prime} f_{m^{\prime}}^{(m)} e^{-i m^{\prime} \omega t} \int_{-\infty}^{t} d t^{\prime} e^{i\left(\epsilon_{m}+m^{\prime} \omega\right)\left(t-t^{\prime}\right)} \\
& \times \mathcal{Y}_{l, m+m^{\prime}}\left(\frac{\mathbf{r}}{2\left(t-t^{\prime}\right)}+\boldsymbol{\alpha}\left(t, t^{\prime}\right)\right) G\left(\mathbf{r}, t ; 0, t^{\prime}\right),
\end{aligned}
$$

where $\alpha\left(t, t^{\prime}\right)$ is defined by Eq. (19).

In the case of circular polarization, for any initial angular momentum $l$ we have only a finite system of $2 l+1$ linear algebraic equations for the coefficients $f_{m^{\prime}}^{(m)}$ and a transcendental equation for $\epsilon$. [This is in contrast to the cases of elliptic or linear polarization, for which one has a system of integro-differential equations for $\left.f^{(m)}(t)\right]$. Moreover, for a given $m$, only the coefficient $f_{m^{\prime}}^{(m)}$ with $m^{\prime}=0$-i.e., $f_{0}^{(m)}$ (which we assume is normalized to unity) _ gives the dominant contribution in most cases. In the perturbative regime $(F \ll 1)$ this fact is obvious since $f_{m^{\prime}}^{(m)} \sim F^{\left|m^{\prime}\right|}$; however, a numerical analysis shows that it is valid also in the strongly nonperturbative regime. Therefore, the coefficients $f_{m^{\prime}}^{(m)}$ with $m^{\prime} \neq 0$ become significant only in the "intermediate" regime, when high-order (in $F$ ) corrections to the lowest-order perturbative result for $\epsilon(F, \omega, \xi= \pm 1)$ should be taken into account. For $s$ states, it is easy to verify that Eqs. (43) and (45) lead to the transcendental equation (30) for the complex quasienergy. We present below explicit equations for $\epsilon$ and the coefficients $f_{m^{\prime}}^{(m)}$ for an initial state with angular momentum $l=1$.

\section{B. Initial state of $\boldsymbol{p}$ symmetry}

The initial state $\psi_{\kappa 1 m=0}(\mathbf{r})$ does not mix with the states $\psi_{\kappa 1 m= \pm 1}(\mathbf{r})$, so that Eq. (45) for this case reduces [using Eqs. (A4) and (A5)] to

$$
\Phi_{1,0}(\mathbf{r}, t)=2 \pi i C_{\kappa 1} r Y_{1,0}(\hat{\mathbf{r}}) \int_{0}^{\infty} e^{i \epsilon \tau} \tau^{-1} G(\mathbf{r}, t ; 0, t-\tau) d \tau .
$$

Expanding this wave function at small $r$ and matching the result to the boundary condition (43) for $l=1, m=m^{\prime}=0$, the resulting transcendental equation for $\epsilon$ may be presented in the form

$$
\begin{aligned}
\frac{1}{a_{1}} & -\frac{r_{1} \epsilon}{2}+i \epsilon^{3 / 2} \\
& =\frac{3}{4} \sqrt{\frac{i}{\pi}} \int_{0}^{\infty} \frac{e^{i \epsilon \tau}\left\{e^{-i u_{p} \tau+4 i\left(u_{p} / \omega\right) \sin ^{2} \omega \tau / 2 / \omega \tau}-1\right\}}{\tau^{5 / 2}} d \tau,
\end{aligned}
$$

which is similar to Eq. (30) for $s$ states. [Note, however, that each of the two terms in the integrand in Eq. (47) has a stronger singularity at $\tau \rightarrow 0$ (i.e., $\sim \tau^{-5 / 2}$ ) than that in Eq. (30) $\left(\sim \tau^{-3 / 2}\right)$. For this reason, the RHS of Eq. (47) cannot be rewritten in terms of the parameter $\left(u_{p}-\epsilon\right)$, as was done in Eq. (30).]

For odd $m, m= \pm 1$, the boundary condition (43) is time dependent, so that the wave function (45) includes two terms, with $m^{\prime}=0,-2$ for $m=1$, and $m^{\prime}=0,2$ for $m=-1$. After simple routine calculations, this wave function may be presented as

$$
\begin{aligned}
\Phi_{1, m}(\mathbf{r}, t)= & 2 \pi i C_{\kappa 1} \sum_{m^{\prime}=-m \pm 1} f_{m^{\prime}}^{(m)} e^{-i m^{\prime} \omega t} \\
& \times \int_{0}^{\infty} e^{i\left(\epsilon+m^{\prime} \omega\right) \tau} G(\mathbf{r}, t ; 0, t-\tau) \\
& \times\left[\frac{r}{\tau} Y_{1, m+m^{\prime}}(\hat{\mathbf{r}})+i \sqrt{\frac{3}{4 \pi}} \frac{F}{\omega} e^{i\left(m+m^{\prime}\right) \omega(t-\tau / 2)}\right. \\
& \left.\times\left(\frac{2 \sin (\omega \tau / 2)}{\omega \tau}-e^{-i\left(m+m^{\prime}\right) \omega \tau / 2}\right)\right] d \tau .
\end{aligned}
$$

Expanding the QQES wave function (48) in a series in $r$ and matching to the boundary condition (43), one obtains a system of two linear homogeneous equations for the coefficients $f_{ \pm 1-m}^{(m)}$ and the quasienergy $\epsilon$ :

$$
\begin{gathered}
\hat{\mathcal{M}}\left(\epsilon_{0}\right) f_{1-m}^{(m)}+\left[\overline{\mathcal{M}}_{-}\left(\epsilon_{-1}\right)-\mathcal{R}\left(\epsilon_{-1}\right)\right] f_{-1-m}^{(m)}=0, \\
{\left[\overline{\mathcal{M}}_{+}\left(\epsilon_{1}\right)-\mathcal{R}\left(\epsilon_{1}\right)\right] f_{1-m}^{(m)}+\hat{\mathcal{M}}\left(\epsilon_{0}\right) f_{-1-m}^{(m)}=0,}
\end{gathered}
$$

where $\mathcal{R}(E)$ is given by Eq. (39), $\epsilon_{k}=\epsilon+(k-m) \omega$, and the explicit forms of the matrix elements $\overline{\mathcal{M}}_{ \pm}$and $\hat{\mathcal{M}}$ are given 

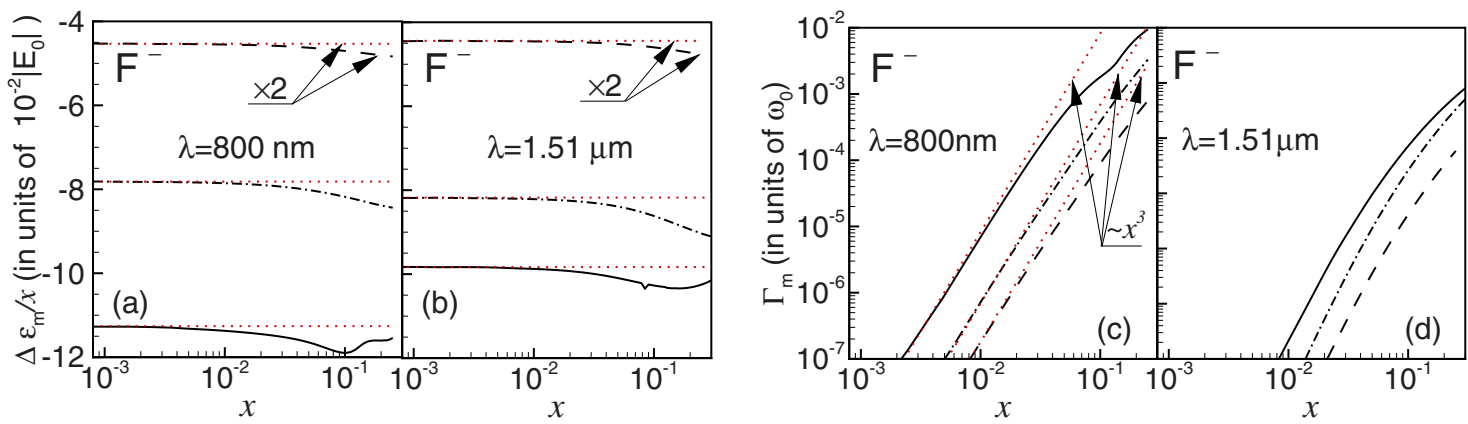

FIG. 11. (Color online) Dependence of the Stark shift $\Delta \epsilon_{m}$ and the detachment rate $\Gamma_{m}$ on the reduced laser intensity $x=I / I_{0}$ for $\mathrm{F}^{-}$in a circularly polarized field $(\xi=+1)$ of wavelengths $\lambda=800 \mathrm{~nm}[(\mathrm{a})$ and (c)] and $\lambda=1.51 \mu \mathrm{m}$ [(b) and (d)]. Solid lines: $m=-1$. Dot-dashed lines: $m=1$. Dashed lines: $m=0$. Dotted lines: lowest-order PT results (cf. Fig. 8). Results for $m=0$ in (a) and (b) have been multiplied by a factor of 2 for better visualization. For $I_{0}$ and $\omega_{0}$, see Fig. 8 .

in Appendix B. After minor transformations, the above results coincide with those obtained in Ref. [31] in a rotating reference frame. The complex quasienergy $\epsilon$ of two states, corresponding to mixed (unperturbed) sublevels with angular momentum projections $m= \pm 1$, is determined as the root of a transcendental equation which is obtained by equating to zero the determinant of the $2 \times 2$ matrix on the LHS of Eq. (49). This determinant has many roots; however, only two of them correspond to the actual values of the quasienergy $\epsilon$-i.e., to those that tend to the binding energy $E_{0}=-1$ as $F \rightarrow 0$.

\section{Stark shift and detachment rate for valence $p$-state negative ions in a circularly polarized field}

As for the case of linear polarization, we provide numerical results for the complex quasienergies of the QQESs (46) and (48) for the case of the $\mathrm{F}^{-}$ion. Note that laser detachment of $\mathrm{F}^{-}$by a circularly polarized field of wavelength $\lambda$ $=1.51 \mu \mathrm{m}(\omega=0.242)$ has been measured recently [51]. To illustrate the qualitative behavior of the complex quasienergies of the field-split sublevels (having different $m$ ) with increasing $F$, in Fig. 11 we present the $F$ dependence of the Stark shifts and the detachment rates for the same laser frequencies as in Fig. 8 for linear polarization. As in the case of an $s$ state in a circularly polarized field, the rates $\Gamma_{m}$ depend smoothly on $F$ even for high frequencies, since the effects of the Wigner threshold law are suppressed for this case (e.g., compared to the results in Fig. 8, they are very smooth, even for $\lambda=800 \mathrm{~nm}$ and $F \approx 0.376$ ). The ordering of the curves for different $m$ in Fig. 11 can be understood in terms of the dipole selection rules for the case of circular polarization; as previously, we assume $\xi=+1$. Generally, for any angular momentum $l$ we have two groups of field-split levels, with even and odd $m$, and in each group the absolute values of $\Delta \epsilon_{m}$ and $\Gamma_{m}$ decrease with increasing $m$. On the one hand, it follows from the dipole selection rules that a minimum value of $m$ ensures also a minimum value of the angular momentum of the detached electron. Therefore, according to the Wigner threshold law [43], the differential rate for detachment to a final state of low energy (up to the energy $\sim 2 U_{p}$ ) will be largest for the smallest $m$. On the other hand, only the low-energy part of the electron spectrum contributes to the total detachment rate, so that we can conclude that the detachment rate for the QQESs with the smallest negative $m$ will be largest. [Note that in general such considerations are valid only for the case in which the photon number of the lowest open detachment channel, $n_{0}$, is higher than the angular momentum $l$ of the initial state. The case of $n_{0}<l$ (which is not relevant to the case of $l=1$ considered in this paper) requires a separate analysis.] The relation between the detachment rates from substates with different parities of the projection $m$ depends on the particular situation and should be analyzed separately for each particular case. As an example, for an initial $p$ state the minimum angular momentum component of the detached electron is the same $(L=2)$ for detachment from $m=1$ and $m=0$ substates. However, due to dipole selection rules, the electrons detached from the substate with $m=0$ cannot be ejected in the polarization plane, where the maximum of the detachment probability is located. Therefore, the detachment rate for the substate with $m=0$ is expected to be less than that for $m=1$ (cf. Table V).

The frequency dependence of the complex quasienergies $\epsilon_{m}$ is shown in Fig. 12 for two laser field intensities. The ordering of the curves for substates with different $m$ follows the rules discussed above. As the frequency tends toward zero, the two levels with $m= \pm 1$ merge to a single one. As discussed with regard to Fig. 9 for the case of linear polarization and in Sec. III B for the case of $s$ states, threshold effects are governed by the Wigner threshold law and are most pronounced for $m=-1$. In this case the absorption of one photon creates a detached electron in an $s$ state of the continuum (whereas for both $m=0$ and 1 , the detached electron has an angular momentum $l=2$ ). It is interesting to note that the threshold peculiarities in $\Gamma_{m}$ become smoother with increasing intensity, while the cusp in $\Delta \epsilon_{m=0}$ at the threshold of the one-photon detachment becomes sharper.

In Table $\mathrm{V}$ we present TDER theory results for the detachment rates of $\mathrm{O}^{-}$and $\mathrm{Br}^{-}$for different magnetic sublevels and laser parameters. These results correspond to three different levels of accuracy: (i) $\Gamma_{m}$ is the exact TDER result, obtained from the solution of Eqs. (47) and (49); (ii) $\widetilde{\Gamma}_{m}$ is an approximate detachment rate obtained from the solution of the system of equations (49) in which the nondiagonal matrix elements $\hat{\mathcal{M}}\left(\epsilon_{0}\right)$ (which mix substates with $m= \pm 1$ ) are neglected, whereas the influence of the parameters $a_{1}$ and $r_{1}$ on 
TABLE V. Detachment rates (in atomic units) for $\mathrm{O}^{-}\left(C_{\kappa 1}^{2}=0.4225\right.$ a.u. $)$ and $\mathrm{Br}^{-}\left(C_{\kappa 1}^{2}=2.22\right.$ a.u. $)$ for right-hand circular polarization $(\xi=+1)$ and different laser field parameters (in scaled units). $(-n) \equiv 10^{-n}$.

\begin{tabular}{|c|c|c|c|c|c|c|}
\hline Ref. & $m=-1$ & $\begin{array}{c}\mathrm{O}^{-} \\
m=0\end{array}$ & $m=1$ & $m=-1$ & $\begin{array}{c}\mathrm{Br}^{-} \\
m=0\end{array}$ & $m=1$ \\
\hline \multicolumn{7}{|c|}{$F=0.145, \omega=0.155$} \\
\hline$\Gamma_{m} C_{\kappa 1}^{-2}$ & $1.26(-7)$ & $3.10(-9)$ & $2.85(-8)$ & $1.66(-7)$ & $4.63(-9)$ & $4.16(-8)$ \\
\hline$\tilde{\Gamma}_{m} C_{\kappa 1}^{-2}$ & $1.32(-7)$ & & $2.85(-8)$ & $1.88(-7)$ & & $4.11(-8)$ \\
\hline$\Gamma_{m}^{K A} C_{\kappa 1}^{-2}$ & $1.37(-7)$ & $3.20(-9)$ & $2.90(-8)$ & $2.08(-7)$ & $4.85(-9)$ & $4.41(-8)$ \\
\hline \multicolumn{7}{|c|}{$F=0.3, \omega=0.155$} \\
\hline$\Gamma_{m} C_{\kappa 1}^{-2}$ & $4.17(-5)$ & $2.54(-6)$ & $2.02(-5)$ & $4.75(-5)$ & $3.73(-6)$ & $2.91(-5)$ \\
\hline$\widetilde{\Gamma}_{m} C_{\kappa 1}^{-2}$ & $4.59(-5)$ & & $2.00(-5)$ & $6.17(-5)$ & & $2.74(-5)$ \\
\hline$\Gamma_{m}^{K A} C_{\kappa 1}^{-2}$ & $4.89(-5)$ & $2.67(-6)$ & $2.07(-5)$ & $7.42(-5)$ & $4.06(-6)$ & $3.14(-5)$ \\
\hline \multicolumn{7}{|c|}{$F=0.15, \omega=0.425$} \\
\hline$\Gamma_{m} C_{\kappa 1}^{-2}$ & $7.58(-6)$ & $1.36(-7)$ & $5.87(-7)$ & $1.01(-5)$ & $2.04(-7)$ & $8.59(-7)$ \\
\hline$\widetilde{\Gamma}_{m} C_{\kappa 1}^{-2}$ & $8.38(-6)$ & & $5.87(-7)$ & $1.22(-0)$ & & $8.59(-7)$ \\
\hline$\Gamma_{m}^{K A} C_{\kappa 1}^{-2}$ & $9.04(-6)$ & $1.44(-7)$ & $6.19(-7)$ & $1.37(-5)$ & $2.18(-7)$ & $9.38(-7)$ \\
\hline \multicolumn{7}{|c|}{$F=0.4, \omega=0.425$} \\
\hline$\Gamma_{m} C_{\kappa 1}^{-2}$ & $4.92(-4)$ & $3.08(-5)$ & $1.37(-4)$ & $5.53(-4)$ & $4.51(-5)$ & $1.89(-4)$ \\
\hline$\widetilde{\Gamma}_{m} C_{\kappa 1}^{-2}$ & $5.71(-4)$ & & $1.37(-4)$ & $7.49(-4)$ & & $1.85(-4)$ \\
\hline$\Gamma_{m}^{K A} C_{\kappa 1}^{-2}$ & $6.55(-4)$ & $3.33(-5)$ & $1.46(-4)$ & $9.93(-4)$ & $5.05(-5)$ & $2.21(-4)$ \\
\hline
\end{tabular}

the matrix elements $\overline{\mathcal{M}}\left(\epsilon_{1}, \xi= \pm 1\right)$ is treated exactly; and (iii) $\Gamma_{m}^{K A}$ is the KA detachment rate [54], which neglects both level mixing and the Stark shift and splitting of the substates with different $m$, so that $\Gamma_{m}^{K A}$ factorizes into an $r_{1}$-independent function and the square of the coefficient $C_{\kappa 1}$. To make clear the dependence of the results in all three cases on the parameter $r_{1}$, we have divided all detachment rates in Table $\mathrm{V}$ by $C_{\kappa 1}^{2}$. Thus, if the entire dependence of $\widetilde{\Gamma}_{m}$ on $r_{1}$ is concentrated only in the factor $C_{\kappa 1}^{2}$, then there will be no difference between $\widetilde{\Gamma}_{m}$ and $\Gamma_{m}^{K A}$. The KA works reasonably well for the QQES with $m=0, \Phi_{1,0}(\mathbf{r}, t)$, which does not involve any admixture of the initially degenerate $m= \pm 1$ substates [cf. Eq. (46)]. As Table V shows, there is only a small contribution of the sublevel with $m=-1$ to $\Gamma_{m=+1}$, so that differences between the KA and TDER results originate mostly from the nonlinear dependence of the complex quasienergy on the effective range $r_{1}$. This result is not surprising, because the admixture of the sublevel having $m=$ -1 with that having $m=+1$ is realized only through the virtual emission of two photons [as is clear from Eq. (48) for $\Phi_{1,+1}(\mathbf{r}, t)$, where the mixing coefficient $f_{-2}^{(+1)}$ is multiplied by the factor $\exp (2 i \omega t)]$. Thus the mixing effect in the rate $\Gamma_{m}$ for the state $\Phi_{1,+1}(\mathbf{r}, t)$ is suppressed, since the admixed component of $\Phi_{1,+1}(\mathbf{r}, t)$ lies deeper in energy than the basic one and requires absorption of two more photons in order to be
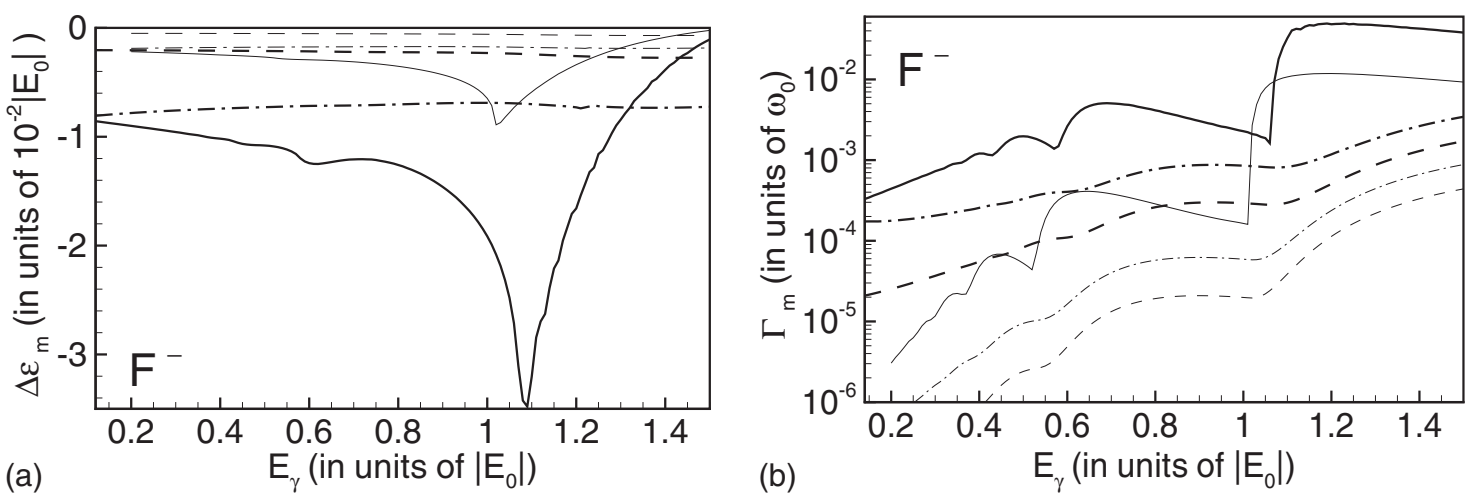

FIG. 12. Dependence of (a) the Stark shift $\Delta \epsilon_{m}$ and (b) the detachment rate $\Gamma_{m}$ on the photon energy $E_{\gamma}=\hbar \omega$ for $\mathrm{F}^{-}$in a circularly polarized field $(\xi=+1)$. Results are given for two laser intensities: $I=1.2 \times 10^{13} \mathrm{~W} / \mathrm{cm}^{2}$ (thick lines) and $3 \times 10^{12} \mathrm{~W} / \mathrm{cm}^{2}($ thin lines). Solid lines: $m=-1$. Dot-dashed lines: $m=1$. Dashed lines: $m=0$. For $\omega_{0}$, see Fig. 8 . 
ionized. On the contrary, for the state $\Phi_{1,-1}(\mathbf{r}, t)$, in which case the admixture of the state having $m=+1$ with that having $m=-1$ happens through the virtual absorption of two photons [since the mixing coefficient $f_{+2}^{(-1)}$ is multiplied by the factor $\exp (-2 i \omega t)]$, the mixing effect is much more important: its neglect gives an inaccuracy up to $10 \%$ or more in $\Gamma_{m=-1}$. (Note that, as discussed above, both coefficients $f_{-2}^{(+1)}$ and $f_{+2}^{(-1)}$ are small compared to $f_{0}^{( \pm 1)}$.) Thus, in general, the sublevel with $m=-1$ has the maximum detachment rate and is more sensitive to different approximations than the other two-i.e., those with $m=0,1$. We emphasize in connection with these general remarks that they apply for the case of right circular polarization, $\xi=+1$; for the case of $\xi=-1$, the situation is inverted. Finally, we note that the largest absolute differences between the TDER and the approximate results occur for the ion with the largest $C_{\kappa 1}$.

\section{CASE OF AN INITIAL $p$ STATE IN AN ELLIPTICALLY POLARIZED FIELD}

\section{A. QQES wave functions and quasienergies}

The case of an elliptically polarized field is the most complicated one in view of the reduced symmetry of the interaction potential $V(\mathbf{r}, t)$ in Eq. (7). Indeed, the only symmetry that exists for this case is that the Hamiltonian in Eq. (7) is invariant with respect to a rotation by the angle $180^{\circ}$ around the direction of the photon wave vector $\mathbf{k}$ (i.e., in the plane of the polarization ellipse), which we assume is directed along the $z$ axis of the reference frame. Therefore, as for the case of $\xi= \pm 1$, the initial bound state $\psi_{\kappa l m}(\mathbf{r})$ evolves into a set of $(2 l+1)$ QQESs $\Phi^{(p)}(\mathbf{r}, t)(p=1,2, \ldots, 2 l+1)$ with different quasienergies $\epsilon_{p}$. However, in contrast to the case of circular polarization, for $0<|\xi|<1$ each quasienergy harmonic $\Phi_{k}^{(p)}(\mathbf{r})$, of $\Phi^{(p)}(\mathbf{r}, t)[\mathrm{cf}$. Eq. (6)] is a mixture of components with different angular momentum projections $m$ (and does not have a fixed angular momentum projection, as in the case of $\xi= \pm 1$ ). Nevertheless, in view of the above-mentioned symmetry, each $\Phi_{k}^{(p)}(\mathbf{r})$ is a combination of angular momentum components $m^{\prime}$ with the same parity of $m^{\prime}$-i.e., $m^{\prime}$ $=0, \pm 2, \ldots$ or $m^{\prime}= \pm 1, \pm 3, \ldots$ Thus $\Phi_{k}^{(p)}(\mathbf{r})$ has definite parity, $(-1)^{m^{\prime}}$, with respect to the substitution $\varphi \rightarrow \varphi+\pi$. Moreover, $\Phi_{k}^{(p)}(\mathbf{r})$ and $\Phi_{k \pm 1}^{(p)}(\mathbf{r})$ have opposite parities, as for the case of circular polarization. This parity depends on the combination of the laser field-mixed $m$ substates $\psi_{\kappa l m}(\mathbf{r})$ (with even or odd $m$ ) to which the harmonic $\Phi_{k=0}^{(p)}(\mathbf{r})$ reduces as $F \rightarrow 0$-i.e., in the limit of the standard (quadratic in $F$ ) Stark effect. As a result, the $(2 l+1)$ equations for $\epsilon_{p}$ and the functions $f^{(p)}(t)$, which enter the boundary condition for $\Phi^{(p)}(\mathbf{r}, t)$ at small $r$, reduce to two separate systems of coupled integro-differential equations corresponding to the mixing of either even- or odd- $m$ substates of $\psi_{\kappa l m}(\mathbf{r})$. To illustrate the QQES results for the case of elliptic polarization, we consider below the simplest example: an initial state of $p$ symmetry.

According to the general considerations above, the boundary condition for each of two QQESs, corresponding to mixed substates (with $m= \pm 1$ ) of an initial state with $l=1$, should involve two functions $f^{(p= \pm 1)}(t)$, which define the small- $r$ behavior of the QQES wave function in the $P$-wave channel with magnetic projections $p= \pm 1$ :

$$
\begin{aligned}
& \int \Phi_{\epsilon}(\mathbf{r}, t) Y_{1 p}^{*}(\hat{\mathbf{r}}) d \Omega_{\mathbf{r}} \\
& \quad \sim \sum_{k}\left[\frac{1}{r^{2}}+\frac{\epsilon+k \omega}{2}+B_{1}(\epsilon+2 k \omega)(r+\cdots)\right] f_{k}^{(p)} e^{-2 i k \omega t},
\end{aligned}
$$

where $f_{k}^{(p)}$ are the Fourier coefficients of functions $f^{(p)}(t)$. The initial state with $l=1$ and $m=0$ is not mixed with $m$ $= \pm 1$ states; thus, the boundary condition at small $r$ for the QQES $\Phi_{\epsilon}(\mathbf{r}, t)$, to which this state evolves in an elliptically polarized field, may be formulated similarly to the case of linear polarization; i.e., it has the form (50) with $p=0$ and involves a single function $f^{(0)}(t)$.

Taking into account the explicit form for the $P$-wave component of the wave function (18) for small $r$ (see Appendix A), it can be verified that the QQES wave functions satisfying the boundary conditions (50) for $p=0$ and $p= \pm 1$ can be composed from three functions $\widetilde{\Phi}^{(0, \pm 1)}(\mathbf{r}, t)$ as follows:

$$
\Phi_{\epsilon}(\mathbf{r}, t)=\left\{\begin{array}{l}
\widetilde{\Phi}^{(0)}(\mathbf{r}, t), \\
{\left[\tilde{\Phi}^{(+1)}(\mathbf{r}, t)+\widetilde{\Phi}^{(-1)}(\mathbf{r}, t)\right] / \sqrt{2},}
\end{array}\right.
$$

where the explicit form of $\widetilde{\Phi}^{(p)}(\mathbf{r}, t)$ is

$$
\begin{aligned}
\widetilde{\Phi}^{(p=0, \pm 1)}(\mathbf{r}, t)= & 4 \pi i C_{\kappa 1} \int_{0}^{\infty} e^{i \epsilon \tau} G(\mathbf{r}, t ; 0, t-\tau) \\
& \times\left[\frac{r Y_{1, p}(\hat{\mathbf{r}})}{2 \tau}+p \sqrt{\frac{3}{8 \pi}} \phi_{p}(t, \tau)\right] f^{(p)}(t-\tau) d \tau
\end{aligned}
$$

and where

$$
\begin{aligned}
\phi_{p}(t, \tau)= & \frac{i F}{\omega \sqrt{2(1+\ell)}}\left\{\frac{2 \sin \frac{\omega \tau}{2}}{\omega \tau}[i(1+\ell) \sin \omega(t-\tau / 2)\right. \\
& +\xi p \cos \omega(t-\tau / 2)]-[i(1+\ell) \sin \omega(t-\tau) \\
& +\xi p \cos \omega(t-\tau)]\}
\end{aligned}
$$

For the case of right circular polarization $(\xi=+1)$, the functions $\widetilde{\Phi}^{(p=0, \pm 1)}(\mathbf{r}, t)$ in Eq. (52) reduce to the functions $\Phi_{1, m=0, \pm 1}(\mathbf{r}, t)$ in Eqs. (46) and (48). For the case of linear polarization, $\phi_{p}(t, \tau)=-\varphi(t, \tau) / 2$, where $\varphi(t, \tau)$ is given by Eq. (37).

Expanding the functions in Eq. (51) in $r$ [cf. Eq. (A12)] and matching their projections on $Y_{1, p}(\hat{\mathbf{r}})$ to the small- $r$ boundary condition (50), one obtains integro-differential equations for the functions $f^{(0)}(t)$ and $f^{( \pm 1)}(t)$ as well as equivalent systems of linear homogeneous equations for the Fourier coefficients $f_{k}^{(p)}$. For $f_{k}^{(0)}$, the equations are 

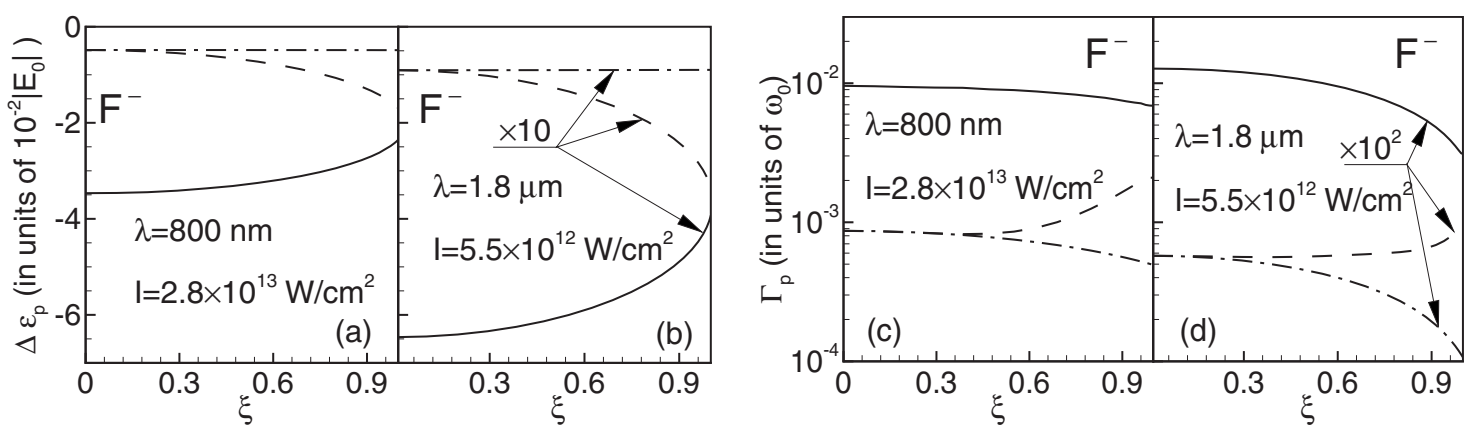

FIG. 13. $\xi$ dependence of the Stark shift (a) and detachment rate (b) for the $\mathrm{F}^{-}$ion. Solid line: the substate with $p=-1$. Dashed line: $p=1$. Dash-dotted line: $p=0$. Results for $\lambda=1.8 \mu \mathrm{m}$ are multiplied by factors 10 and $10^{2}$ for the Stark shift and detachment rate, respectively. For $\omega_{0}$, see Fig. 8 .

$$
\mathcal{R}(E) f_{k}^{(0)}=\sum_{k^{\prime}} M_{k, k^{\prime}}^{(0)}(\epsilon) f_{k^{\prime}}^{(0)},
$$

while for mixed states with $m= \pm 1$, we have two coupled systems of equations for the coefficients $f_{k}^{( \pm 1)}$ :

$$
\begin{gathered}
\mathcal{R}(E) f_{k}^{(-1)}=\sum_{k^{\prime}}\left[\bar{M}_{k, k^{\prime}}(-\xi) f_{k^{\prime}}^{(-1)}+\hat{M}_{k, k^{\prime}}(-\xi) f_{k^{\prime}}^{(+1)}\right], \\
\mathcal{R}(E) f_{k}^{(+1)}=\sum_{k^{\prime}}\left[\bar{M}_{k, k^{\prime}}(\xi) f_{k^{\prime}}^{(+1)}+\hat{M}_{k, k^{\prime}}(\xi) f_{k^{\prime}}^{(-1)}\right],
\end{gathered}
$$

where $E=\epsilon+2 k \omega$, and $\mathcal{R}(E)$ is given by Eq. (39). The explicit forms of the matrix elements in Eqs. (54) and (55) are given by Eqs. (B6)-(B8), which allow one to verify the following symmetry relations:

$$
\begin{gathered}
\bar{M}_{k, k^{\prime}}(\epsilon, \xi)=\bar{M}_{k^{\prime}, k}(\epsilon, \xi), \\
\hat{M}_{k, k^{\prime}}(\epsilon, \xi)=\hat{M}_{k^{\prime}, k}(\epsilon,-\xi) .
\end{gathered}
$$

Numerical analysis over a wide range of laser frequencies, intensities, and ellipticities shows that the determinant of the system (54) vanishes only for the unique value of $\epsilon$ which tends to the unperturbed energy $E_{0}=-1$ as $F \rightarrow 0$. Furthermore, all roots equal $\epsilon$ modulo $\omega$. A similar analysis shows that the determinant of Eq. (55) vanishes for two different values of $\epsilon$, which both tend to $E_{0}$ as $F \rightarrow 0$. Thus Eq. (55) yields a set of two different quasienergies $\epsilon_{ \pm 1}$ and corresponding eigenvectors $f_{k}^{( \pm 1)}\left(\epsilon_{+1}\right)$ and $f_{k}^{( \pm 1)}\left(\epsilon_{-1}\right)$ that describe two Stark-split quasistationary states corresponding to linear combination of initial substates $\psi_{\kappa l m}(\mathbf{r})$ with $m= \pm 1$.

Finally, we explain how the complex quasienergy results for the cases of circular and linear polarizations follow from the general equations (54) and (55). For $\xi= \pm 1 \quad(\ell=0)$, the explicit forms of the matrix elements $\bar{M}_{k, k^{\prime}}, \hat{M}_{k, k^{\prime}}$, and $M_{k, k^{\prime}}^{(0)}(\epsilon)$ (cf. Appendix B) simplify drastically. As a result, the system (55) reduces to an infinite set of equivalent independent subsystems involving only two linear equations, similar to the system (49) for $m= \pm 1$, while Eq. (54) reduces to Eq. (47) for $m=0$. For the case of linear polarization $(\xi$ $=0)$, the matrix elements $M_{k, k^{\prime}}^{(0)}(\epsilon)$ in Eq. (54) coincide with $M_{k, k^{\prime}}(\epsilon)$ in Eq. (38) (cf. Appendix B). Thus the solution of

the system (54) yields the same values for the quasienergy $\epsilon^{(0)}$ and the coefficients $f_{k}^{(0)}$ as for the degenerate substates with $|m|=1$ in a linearly polarized field (in which case the quantization axis is directed along the laser polarization). For $\xi=0$, the matrix elements in Eq. (55) become simpler [cf. Appendix B and the symmetry relation (57)], so that the system (55) has two possible solutions $\left\{f^{(+1)}(t), f^{(-1)}(t)\right\}$ : the "symmetric" solution, in which $f^{(+1)}(t)=f^{(-1)}(t) \equiv f^{(+)}(t)$, and the "antisymmetric" one, in which $f^{(+1)}(t)=-f^{(-1)}(t) \equiv f^{(-)}(t)$. Substituting both these solutions into Eq. (55), we obtain two independent systems for the Fourier coefficients of the functions $f^{(+)}(t)$ and $f^{(-)}(t)$ : the system for $f_{k}^{(-)}$is equivalent to that in Eq. (38) for $m=0$, while the system for $f_{k}^{(+)}$coincides with the system (54) for $\xi=0$ and is equivalent to Eq. (38) for $m= \pm 1$.

\section{B. Ellipticity dependence of the Stark shift and detachment rates for a $p$ state $\left(\mathrm{F}^{-}\right.$ion)}

The ellipticity dependence of the Stark shift $\left(\Delta \epsilon_{p}\right)$ and the detachment rate $\left(\Gamma_{p}\right)$, defined by

$$
\epsilon_{p}=-1+\Delta \epsilon_{p}-i \Gamma_{p} / 2
$$

are shown in Fig. 13 for the three split substates of the $\mathrm{F}^{-}$ ion. For $\xi=0$, two of the three levels are degenerate-i.e., those with angular momentum projections $m= \pm 1$ on the laser polarization axis. This degeneracy is removed with increasing $\xi$. The position of one of these degenerate levels (corresponding to the state with $m=0$ in an elliptically polarized field) is essentially independent of $\xi$, while its decay rate gradually decreases with increasing $\xi$. The positions of the other two levels, which are mixed for $|\xi| \neq 0$, as well as their corresponding detachment rates become closer to each other as $|\xi|$ increases. The dependence of $\Delta \epsilon_{p}$ and $\Gamma_{p}$ on $\xi$ is monotonic with a slope whose magnitude increases gradually, becoming most pronounced at $|\xi|=1$. As discussed previously, the threshold effects in the intensity dependence of the complex quasienergy are most prominent for linear polarization (cf. Fig. 8) and disappear for circular polarization (cf. Fig. 11). For $0<|\xi|<1$, the threshold peculiarities still exist (see Fig. 14), but are relatively smooth compared to the case of $\xi=0$ due to the decreasing population of the $S$-wave components in the final state of the detached electron as $\xi$ increases. 

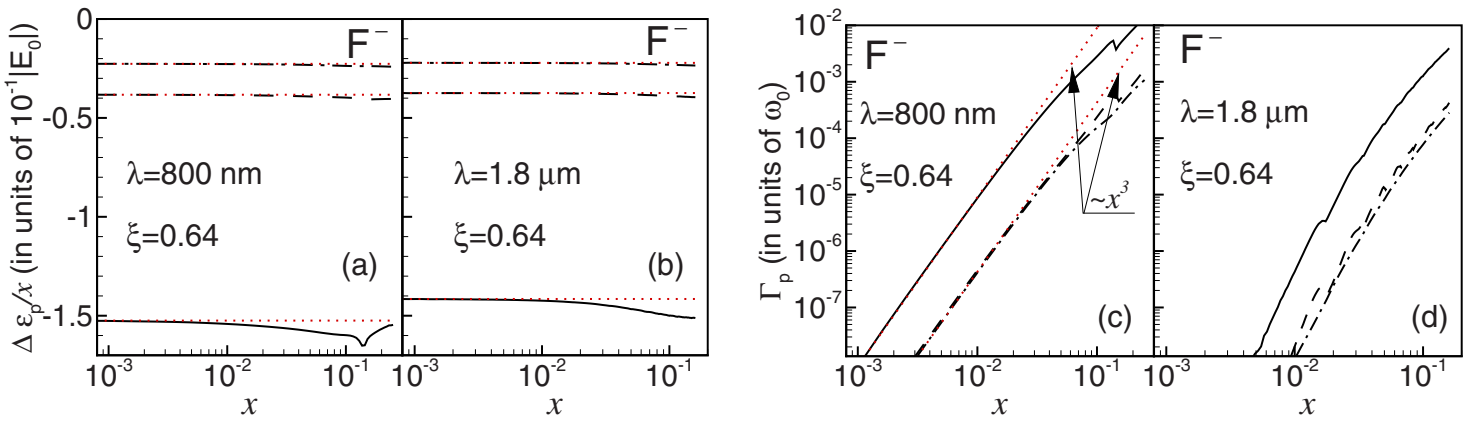

FIG. 14. (Color online) The same as in Fig. 11, but for the elliptic polarization $\xi=0.64$. Solid line: $p=-1$. Dashed line: $p=1$. Dash-dotted line: $p=0$.

\section{Ellipticity dependence of the Fourier coefficients $f_{k}^{(p)}$ for a $p$ state $\left(\mathbf{F}^{-}\right.$ion $)$}

In Fig. 15 we present the spectra of coefficients $f_{k}^{(-1)}\left(\epsilon_{ \pm 1}\right)$ and $f_{k}^{(0)}\left(\epsilon_{0}\right)$ as functions of the degree of circular polarization $\xi$ for $0 \leqslant \xi \leqslant 1$. [Our numerical analysis shows that the behaviors of the two other coefficients $f_{k}^{(+1)}\left(\epsilon_{ \pm 1}\right)$ are similar to those for $f_{k}^{(-1)}(\epsilon \pm 1)$.] For $\xi=0$, the coefficients $f_{k}^{(-1)}\left(\epsilon_{-1}\right)$ coincide with the coefficients $f_{k}^{(m=0)}$ for the case of linear polarization, while the coefficients $f_{k}^{(-1)}\left(\epsilon_{+1}\right)$ are equal to $f_{k}^{(0)}\left(\epsilon_{0}\right)$ and coincide with the coefficients $f_{k}^{(|m|=1)}$ for the case of linear polarization [cf. Eq. (38)]. For the opposite limiting case $\xi=1$, the sets of coefficients $f_{k}^{(0)}\left(\epsilon_{0}\right)$ and $f_{k}^{( \pm 1)}\left(\epsilon_{ \pm 1}\right)$ reduce to $\delta_{k, 0}$, while the set $f_{k}^{( \pm 1)}\left(\epsilon_{\mp 1}\right)$ reduces to $\delta_{k, \pm 1}$. Interestingly, the reduction of these sets of Fourier coefficients to Kronecker $\delta$ 's happens in a very narrow interval of $\xi$ near $\xi=1$ (e.g., for the laser parameters in Fig. 15, $f_{0}^{(-1)}\left(\epsilon_{+1}\right)=0.0454$ for $\left.\xi=0.99995\right)$. Thus the transition to the case of pure circular polarization cannot be properly presented on the scale of Fig. 15, where even for $\xi=0.98$ some signatures of plateau structures in the coefficients $f_{k}^{(-1)}\left(\epsilon_{ \pm 1}\right)$ still exist.

The dependence of the coefficients $f_{k}^{(-1)}\left(\epsilon_{-1}\right)$ and $f_{k}^{(0)}\left(\epsilon_{0}\right)$ on $\xi$ in Fig. 15 is very similar to that for the coefficients $f_{k}$ for an $s$ state (cf. Fig. 7); i.e., both the height and the extent (in $k$ ) of the plateau structures have maxima at $\xi=0$ and gradually decrease with increasing $\xi$, with a sharp downturn at $\xi \approx 1$. The smallness of the coefficients $f_{k}^{(0)}\left(\epsilon_{0}\right)$ compared to $f_{k}^{(-1)}\left(\epsilon_{-1}\right)$ is similar to that between the coefficients $f_{k}^{(|m|=1)}$ and $f_{k}^{(m=0)}$ for the case of linear polarization. The ellipticity dependence of the coefficients $f_{k}^{(-1)}\left(\epsilon_{+1}\right)$, as well as that for $f_{k}^{(+1)}\left(\epsilon_{+1}\right)$ (not shown), however, exhibits quite unusual behavior for small $\xi$ : starting from $\xi=0$ [where the two sets coincide with $f_{k}^{(0)}\left(\epsilon_{0}\right)$, these coefficients increase by as much as an order of magnitude with increasing $\xi$ (up to $\xi \approx 0.3$ ). As a result, the maxima in the height and extent of the plateau structures for the coefficients $f_{k}^{( \pm 1)}\left(\epsilon_{+1}\right)$ are achieved at nonzero values of $\xi$. Plateau features in the spectra of the Fourier coefficients $f_{k}^{( \pm 1,0)}$ are closely connected to the intensity of rescattering effects (cf. the discussion in Sec. III B). Thus one can conclude that in laser interactions with a bound electron having nonzero angular momentum, electron rescattering in a laser field with nonzero ellipticity may be even more effective than for the case of pure linear polarization.

A detailed mathematical analysis of the trends in the ellipticity dependence of the coefficients $f_{k}^{( \pm 1)}\left(\epsilon_{+1}\right)$ that are exhibited in Fig. 15 is presented in Appendix C.

\section{SUMMARY AND CONCLUSIONS}

The main results of this paper are the following: (i) The formal development of a general nonperturbative method (the TDER theory) for the analytic solution of the timedependent Schrödinger equation (7) for the complex quasienergy $\epsilon$ and the quasistationary wave function $\Phi_{\epsilon}(\mathbf{r}, t)$, corresponding to an initially bound state $\psi_{\kappa l m}(\mathbf{r})=R_{\kappa l}(r) Y_{l m}(\hat{\mathbf{r}})$ of an electron in a short-range potential $U(r)$ of radius $r_{c}$ (cf.
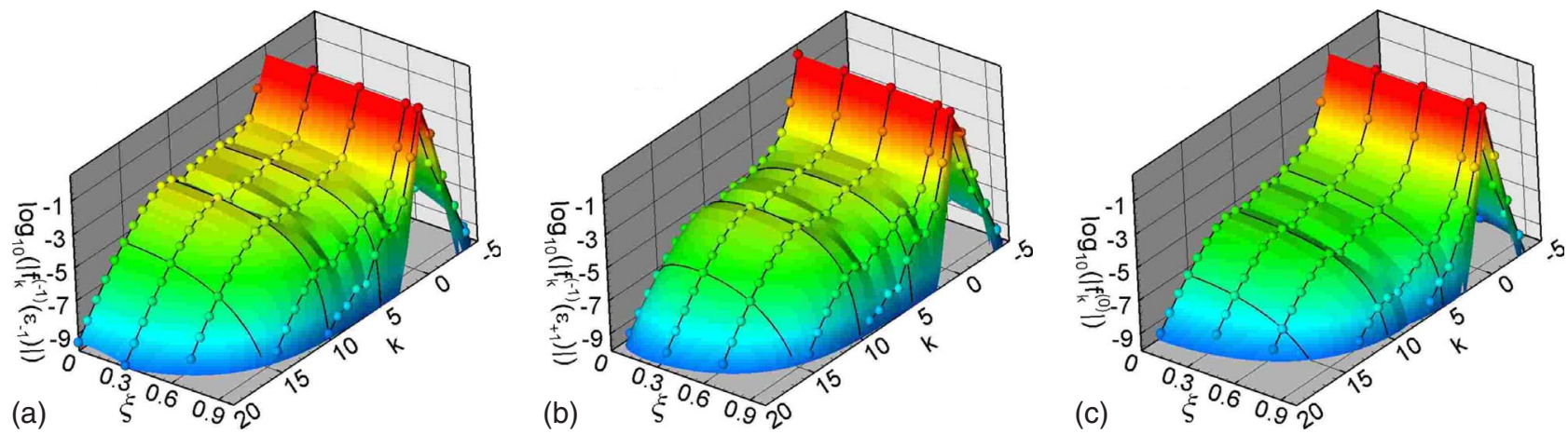

FIG. 15. (Color online) Dependence of coefficients $f_{k}^{(-1)}\left(\epsilon_{+1}\right), f_{k}^{(-1)}\left(\epsilon_{-1}\right)$, and $f_{k}^{(0)}$ on both $k$ and $\xi$ for $\mathrm{F}^{-}$at $I=1.23 \times 10^{13} \mathrm{~W} / \mathrm{cm}^{2}$ and $\lambda=1.8 \mu \mathrm{m}$. Points corresponding to fixed $\xi(\xi=0,0.3,0.6,0.9,0.98)$ and lines corresponding to fixed $k(k=5,10,15)$ have been added for better data visualization. 
Sec. II); (ii) derivation of explicit equations for $\epsilon$ and $\Phi_{\epsilon}(\mathbf{r}, t)$ for the particular cases of initial $s$ and $p$ states (which correspond, in particular, to negative ions with $s$ and $p$ valence electrons); (iii) numerical analysis of the laser frequency and intensity dependence of $\epsilon$ and the Fourier coefficients $f_{k}^{(m)}$ of $\Phi_{\epsilon}(\mathbf{r}, t)$ at small $r$ for the cases of linear, circular, and elliptic laser field polarizations (cf. Secs. III-VI); and (iv) application of the TDER theory to the calculation of total laser detachment rates for a number of negative ions: $\mathrm{H}^{-}, \mathrm{O}^{-}$, the alkali-metal negative ions, and the halogen negative ions (cf. Tables II, IV, and V). In what follows we make some concluding remarks on this work.

The key idea for handling Eq. (7) in the TDER theory is that the potentials $U(r)$ and $V(\mathbf{r}, t)$ act in essentially nonoverlapping coordinate ranges-i.e., $r \leqq r_{c}$ for the former and $r$ $\gtrsim \kappa^{-1}$ for the latter, where $\kappa^{-1}$ is the "radius" of the bound state $\psi_{\kappa l m}(\mathbf{r})$ and $\kappa r_{c} \ll 1$. Thus the solution of Eq. (7) at small $r$ may be replaced by the appropriate boundary condition for $\Phi_{\epsilon}(\mathbf{r}, t)$ [involving a periodic in time function $f_{\epsilon}^{(m)}(t)$ ] on the edge $\left(r_{c}\right)$ of a potential well $U(r)$ [cf. Eq. (12)], while the solution outside the potential $U(r)$ may be constructed in explicit analytic form in terms of the Volkov Green's function [cf. Eq. (18)]. Matching this solution at $r_{c}<r<\kappa^{-1}$ to the boundary condition (in which the standard effective range parametrization for the $\epsilon$-dependent factor is used) yields the key equations of TDER theory-i.e., the homogeneous integro-differential equations for $f_{\epsilon}^{(m)}(t)$ and $\epsilon$. The four-dimensional Schrödinger equation (7) is thus reduced to a one-dimensional eigenvalue problem.

These results are the basic ingredients for an essentially analytical, self-consistent theory for an $s$ or $p$ electron in a short-range potential interacting with a strong elliptically polarized laser field. When applying this theory to describe the interaction of a negative ion with a laser field, only two parameters are necessary to specify the particular ion: the electron affinity $E_{0}$ and the asymptotic coefficient $C_{\kappa l}$ of the wave function $R_{\kappa l}(r)$ at $r \gg \kappa^{-1}$. We have applied this TDER theory to analyze the dependence of the complex quasienergy (i.e., the Stark shift and the detachment rate) of an initially degenerate state $\psi_{\kappa l m}(\mathbf{r})$ of a negative ion on both the laser parameters (i.e., intensity, frequency, and ellipticity) and the initial-state $s$ or $p$ symmetry. As shown, the behavior of the function $\epsilon=\epsilon(F, \omega)$ depends strongly on this symmetry. In fact, we find that the frequency and intensity dependence of both the real and imaginary parts of $\epsilon$ are much more sensitive to the initial-state symmetry than to the particular negative ions having the same symmetry. This sensitivity is not surprising, since all irregularities of an analytic function [such as $\epsilon(F, \omega)$ ] are defined by its points of nonanalyticity. For $\epsilon(F, \omega)$, these points correspond to the thresholds of the closing or opening of some $n$-photon detachment channel due to variation of the intensity and/or the frequency. Since the Wigner threshold law is sensitive to the angular momentum of the detaching electron, it causes differences in the $\omega$ and $F$ dependences of the complex quasienergy for ions with $s$-or $p$-state valence electrons.

Finally, we note that our analysis of Eqs. (54) and (55) for the most general case of an elliptic polarization allows one to conclude that the number of quasistationary states remains the same as the number of degenerate in $m$ substates of the initial bound state $\psi_{\kappa l m}(\mathbf{r})$-i.e., $(2 l+1)$. This statement agrees with results of more formal analyses concerning the number of bound levels for an electron in a threedimensional (3D) short-range potential in the presence of a strong laser field [55]. [Note that in Ref. [55] only the timeindependent part of the total Hamiltonian in the oscillating ("Kramers-Henneberger") reference frame was taken into account, whereas in our approach our conclusion is based on the exact solutions.] For the 1D case, the number of quasienergy levels is not conserved owing to the occurrence of socalled laser-induced states with increasing laser intensity [56]. Thus we surmise that these states are artifacts inherent to $1 \mathrm{D}$ models and cannot be used as a tool for analyzing strong field phenomena in real 3D systems bound by a shortrange potential.

\section{ACKNOWLEDGMENTS}

This work was supported in part by RFBR Grant No. 07-02-00574 and NSF Grant No. PHY-0601196. We acknowledge a number of useful discussions with Evgeny A. Pronin. M.V.F. and N.L.M. also gratefully acknowledge the hospitality of The University of Nebraska, where part of this paper was prepared.

\section{APPENDIX A: SMALL- $r$ BEHAVIOR OF THE QQES WAVE FUNCTION $\Phi_{\epsilon}(\mathbf{r}, t)$ IN EQ. (18)}

Before expanding the wave function (18) in $r$, we present first its explicit form at $\mathbf{F}(t)=0$. By putting in Eq. (18) $\mathbf{F}(t)$ $=0$ and substituting $f^{(m)}(t)=1$ we find (in scaled units, which are used through this appendix)

$$
\begin{aligned}
\left.\Phi_{\epsilon}(\mathbf{r}, t)\right|_{F=0} \equiv & \widetilde{\phi}_{l, m}(\mathbf{r} ; \epsilon) \\
= & -4 \pi(-i)^{l} C_{\kappa l} Y_{l, m}(\hat{\mathbf{r}}) \\
& \times \int_{-\infty}^{t} d t^{\prime}\left[\frac{r}{2\left(t-t^{\prime}\right)}\right]^{l} \\
& \times G_{0}\left(\mathbf{r}, t ; 0, t^{\prime}\right) \exp \left[i \epsilon\left(t-t^{\prime}\right)\right] \\
= & i C_{\kappa l}(\sqrt{\boldsymbol{\epsilon}})^{l} \sqrt{\frac{\pi \sqrt{\epsilon}}{2 r}} H_{l+1 / 2}^{(1)}(\sqrt{\epsilon} r) Y_{l, m}(\hat{\mathbf{r}}),
\end{aligned}
$$

where the Hankel function of the first kind, $H_{l+1 / 2}^{(1)}(\sqrt{\epsilon} r)$, appears after integration with respect to $\tau=t-t^{\prime}$, taking into account the Feynman representation (14) for the freeelectron Green's function $G_{0}$. Using the known expansions of $H_{l+1 / 2}^{(1)}(z)$ for small and large arguments [57], we obtain the behaviors of $\widetilde{\phi}_{l m}(\mathbf{r} ; \epsilon)$ for both small $r$,

$$
\begin{aligned}
\widetilde{\phi}_{l, m}(\mathbf{r} ; \epsilon)= & C_{\kappa l}\left[\frac{(2 l-1) ! !}{r^{l+1}}\left(1+\frac{\epsilon r^{2}}{2(2 l-1)}+\cdots\right)\right. \\
& \left.+i \frac{\epsilon^{l+1 / 2} r^{l}}{(2 l+1) ! !}\left(1-\frac{\epsilon r^{2}}{2(2 l+3)}+\cdots\right)\right] Y_{l, m}(\hat{\mathbf{r}}),
\end{aligned}
$$

and large $r$, 


$$
\widetilde{\phi}_{l, m}(\mathbf{r} ; \epsilon)=C_{\kappa l}(-i \sqrt{\boldsymbol{\epsilon}})^{l} \frac{\exp (i \sqrt{\epsilon})}{r} Y_{l, m}(\hat{\mathbf{r}})+\cdots
$$

To extract the terms regular and irregular in $r$ in the expansion of the function $\Phi_{\epsilon}(\mathbf{r}, t)$ at small $r$, we start with the expansion of the function $\mathcal{Y}_{l, m}\left(\frac{\mathbf{r}}{2\left(t-t^{\prime}\right)}+\boldsymbol{\alpha}\left(t, t^{\prime}\right)\right) G\left(\mathbf{r}, t ; 0, t^{\prime}\right)$ [cf. the integrand in Eq. (18) for $\Phi_{\epsilon}(\mathbf{r}, t)$ ] in both $r$ and $\tau$ $=t-t^{\prime}$ variables. First, we represent the solid harmonic $\mathcal{Y}_{l, m}(\mathbf{a})$ as the irreducible tensor product of rank $l$ composed of the rank-1 tensors a [35]:

$$
\begin{aligned}
\mathcal{Y}_{l, m} & \left(\frac{\mathbf{r}}{2\left(t-t^{\prime}\right)}+\boldsymbol{\alpha}\left(t, t^{\prime}\right)\right) \\
& =\sqrt{\frac{(2 l+1) ! !}{4 \pi l !}}\left\{\frac{\mathbf{r}}{2\left(t-t^{\prime}\right)}+\boldsymbol{\alpha}\left(t, t^{\prime}\right)\right\}_{l, m},
\end{aligned}
$$

which can be further simplified using Eq. (44) of Ref. [58],

$$
\left\{c_{1} \mathbf{a}_{1}+c_{2} \mathbf{a}_{2}\right\}_{l, m}=\sum_{k=0}^{l}\left(\begin{array}{l}
l \\
k
\end{array}\right) c_{1}^{l-k} c_{2}^{k}\left\{\left\{\mathbf{a}_{1}\right\}_{l-k} \otimes\left\{\mathbf{a}_{2}\right\}_{k}\right\}_{l, m},
$$

where $c_{1,2}$ are some scalars and $\left(\begin{array}{l}l \\ k\end{array}\right)$ is the binomial coefficient. Taking into account the behaviors of the functions $R$, $M$, and $\alpha\left(t, t^{\prime}\right)$ [see Eqs. (14) and (18)] at small $r$ and $\tau$, we find that expansion of the $l$-wave component of the product $\mathcal{Y}_{l, m}\left(\frac{\mathbf{r}}{2\left(t-t^{\prime}\right)}+\boldsymbol{\alpha}\left(t, t^{\prime}\right)\right) G\left(\mathbf{r}, t ; 0, t^{\prime}\right)$ in both $r$ and $\tau$ can be presented as follows:

$$
\begin{aligned}
& \int \mathcal{Y}_{l, m}\left(\frac{\mathbf{r}}{2\left(t-t^{\prime}\right)}+\boldsymbol{\alpha}\left(t, t^{\prime}\right)\right) G\left(\mathbf{r}, t ; 0, t^{\prime}\right) Y_{l, m}^{*}(\hat{\mathbf{r}}) d \Omega_{\mathbf{r}} \\
& =r^{l} \sum_{s=0}^{l} \frac{a_{s}}{\tau^{l-3 s+3 / 2}}+\cdots,
\end{aligned}
$$

where $a_{s}$ are some coefficients independent of $r$ and $\tau$.

Restricting our analysis to the case of $l \leqslant 2$, it can be seen from the expansion (A6) that the terms with $s>0$ are integrable with respect to $\tau$ at $\tau=0$ and therefore contribute only to the regular $\left(\sim r^{l}\right)$ part of the expansion of $\Phi_{\epsilon}(\mathbf{r}, t)$ in $r$, while the term with $s=0$ involves a nonintegrable singularity at $\tau=0$ and thus contributes to both the irregular and regular parts of the expansion of $\Phi_{\epsilon}(\mathbf{r}, t)$ in $r$. Moreover, the term with $s=0$ in Eq. (A6) originates from the first term (with $k$ $=0$ ) of the sum (A5), where $\mathbf{a}_{1} \sim \mathbf{r}\left[2\left(t-t^{\prime}\right)\right]^{-1}$. Therefore, in order to estimate the contribution of this term to the expansion of $\Phi_{\epsilon}(\mathbf{r}, t)$ at small $r$ more precisely, we must analyze the following integral:

$$
\begin{aligned}
\widetilde{\Phi}_{\epsilon}(\mathbf{r}, t)= & -4 \pi(-i)^{l} C_{\kappa l} \int_{-\infty}^{t} d t^{\prime} e^{i \epsilon\left(t-t^{\prime}\right)} \\
& \times f\left(t^{\prime}\right) \mathcal{Y}_{l, m}\left(\frac{\mathbf{r}}{2\left(t-t^{\prime}\right)}\right) G\left(\mathbf{r}, t ; 0, t^{\prime}\right),
\end{aligned}
$$

where $f\left(t^{\prime}\right)$ is a periodic function of $t^{\prime}$. For $\mathbf{F}(t)=0$, the function $\widetilde{\Phi}_{\epsilon}(\mathbf{r}, t)$ reduces to a superposition of functions $\widetilde{\phi}_{l, m}(\mathbf{r} ; \epsilon)$ [cf. Eq. (A1)]:

$$
\widetilde{\Phi}_{\epsilon}(\mathbf{r}, t) \rightarrow \widetilde{\Phi}_{\epsilon}^{(0)}(\mathbf{r}, t)=\sum f_{n} e^{-i n \omega t} \widetilde{\phi}_{l, m}(\mathbf{r} ; \epsilon+n \omega)
$$

where $f_{n}$ are the Fourier coefficients of $f(t)$. By adding and subtracting the function $\tilde{\Phi}_{\epsilon}^{(0)}(\mathbf{r}, t)$ on the RHS of Eq. (A7), we obtain

$$
\begin{aligned}
\tilde{\Phi}_{\epsilon}(\mathbf{r}, t)= & \tilde{\Phi}_{\epsilon}^{(0)}(\mathbf{r}, t)-4 \pi C_{\kappa l}(-i r)^{l} Y_{l, m}(\hat{\mathbf{r}}) \sum_{n} f_{n} e^{-i n \omega t} \\
& \times \int_{0}^{\infty} e^{i(\epsilon+n \omega) \tau} \frac{\left[G(\mathbf{r}, t ; 0, t-\tau)-G_{0}(\mathbf{r}, t ; 0, t-\tau)\right]}{(2 \tau)^{l}} d \tau .
\end{aligned}
$$

To obtain the expansion of $\widetilde{\Phi}_{\epsilon}(\mathbf{r}, t)$ in $r$ up to terms $\sim r^{l}$, we expand $\widetilde{\Phi}_{l}^{(0)}(\mathbf{r}, t)$ in Eq. (A9) in $r$ using Eqs. (A8) and (A2) and set $\mathbf{r}=0$ in arguments of the Green's functions $G$ and $G_{0}$ in the second term on the RHS of Eq. (A9) (because this term already involves the factor $r^{l}$ ). After some simple algebra, we obtain the following expansion of $\widetilde{\Phi}_{\epsilon}(\mathbf{r}, t)$ at small $r$ :

$$
\begin{aligned}
\tilde{\Phi}_{\epsilon}(\mathbf{r}, t)= & C_{\kappa l} Y_{l m}(\hat{\mathbf{r}})\left(\frac{f(t)(2 l-1) ! !}{r^{l+1}}+\cdots\right. \\
& +r^{l} \sum_{n} f_{n} e^{-i n \omega t}\left[i \frac{(\epsilon+n \omega)^{l+1 / 2}}{(2 l+1) ! !}+\frac{1}{(2 i)^{l}}\right. \\
& \left.\left.\times \int_{0}^{\infty} e^{i(\epsilon+n \omega) \tau} \frac{e^{i S(t, t-\tau)}-1}{\sqrt{4 \pi i} \tau^{l+3 / 2}} d \tau\right]+\cdots\right),
\end{aligned}
$$

where the classical action $S(t, t-\tau)$ is defined by Eq. (23). The wave function $\Phi_{\epsilon}(\mathbf{r}, t)$ at small $r$ differs from $\widetilde{\Phi}_{\epsilon}(\mathbf{r}, t)$ in Eq. (A10) only by terms $\sim r^{l}$, which correspond to terms with $s>0$ in Eq. (A6) [or to terms with $k>0$ in the sum (A5)]. Therefore, $\Phi_{\epsilon}(\mathbf{r}, t)$ can be matched to the boundary condition (12), which has the same structure in $r$ as that in Eq. (A10).

Below we present the explicit small- $r$ forms of $\Phi_{\epsilon}(\mathbf{r}, t)$ for $l=0$ and 1 , which are used in the main text. For the case of $l=0$, the sum in Eq. (A5) includes only the term with $k$ $=0$, so that the expansion of $\Phi_{\epsilon}(\mathbf{r}, t)$ is given by Eq. (A10) with $l=0$, which after some transformations may be presented as follows:

$$
\begin{aligned}
\Phi_{\epsilon}(\mathbf{r}, t)= & \frac{C_{\kappa 0}}{\sqrt{4 \pi}}\left[\frac{f(t)}{r}\right. \\
& \left.+\int_{0}^{\infty} \frac{e^{i \epsilon \tau+i S(t, t-\tau)} f(t-\tau)-f(t)}{\sqrt{4 \pi i} \tau^{3 / 2}} d \tau+\cdots\right] .
\end{aligned}
$$

For $l=1$, the sum (A5) involves two terms, with $k=0$ and $k$ $=1$; thus, the results become more complicated and we present below only the $p$-wave component of $\Phi_{\epsilon}(\mathbf{r}, t)$ at small $r$ up to terms of order $r$ : 


$$
\begin{aligned}
\left.\Phi_{\epsilon}(\mathbf{r}, t)\right|_{r \rightarrow 0}= & C_{\kappa 1} Y_{1 m}(\hat{\mathbf{r}}) \sum_{n} f_{n}^{(m)} e^{-i n \omega t}\left[\frac{1}{r^{2}}+\frac{\epsilon+n \omega}{2}+r\left(i \frac{(\epsilon+n \omega)^{3 / 2}}{3}-\int_{0}^{\infty} e^{i(\epsilon+n \omega) \tau} \frac{e^{i S(t, t-\tau)}-1}{\sqrt{-16 \pi i} \tau^{5 / 2}} d \tau\right)\right] \\
& +C_{\kappa 1}\left\{\begin{array}{l}
\delta_{m, 0} r Y_{10}(\hat{\mathbf{r}}) \int_{0}^{\infty} \frac{e^{i \epsilon \tau+i S(t, t-\tau)}}{\sqrt{64 \pi i} \tau^{3 / 2}} \varphi(t-\tau,-\tau) \varphi(t, \tau) f^{(0)}(t-\tau) d \tau \\
m r \int_{0}^{\infty} \frac{e^{i \epsilon \tau+i S(t, t-\tau)}}{\sqrt{16 \pi i} \tau^{3 / 2}} \phi_{m}(t, \tau)\left[Y_{1,1}(\hat{\mathbf{r}}) \phi_{-1}(t-\tau,-\tau)-Y_{1,-1}(\hat{\mathbf{r}}) \phi_{1}(t-\tau,-\tau)\right] f^{(m)}(t-\tau) d \tau
\end{array}\right\},
\end{aligned}
$$

where the functions $\varphi(t, \tau)$ and $\phi_{m= \pm 1}(t, \tau)$ are given by Eqs. (37) and (53), respectively. The upper line in braces in Eq. (A12) corresponds to the case of linear polarization (where the quantization axis is directed along the polarization vector), while the bottom line corresponds to the case of elliptic polarization (where the quantization axis is directed along the photon wave vector).

\section{APPENDIX B: EXPLICIT FORMS OF THE MATRIX ELEMENTS IN EQUATIONS FOR $\epsilon$}

In this appendix we present the explicit forms of the matrix elements (such as $M_{k, k^{\prime}}$ ) that are involved in the equations for the complex quasienergy $\epsilon$ for $s$ states [cf. Eq. (26)] and $p$ states [cf. Eqs. (38), (49), (54), and (55)]. For concise presentation of these matrix elements, we define the following functions:

$$
\begin{gathered}
z(\tau)=\frac{u_{p}}{\omega}\left(\sin \omega \tau-\frac{4 \sin ^{2}(\omega \tau / 2)}{\omega \tau}\right), \\
\lambda(\tau)=\frac{u_{p}}{\omega}\left(\omega \tau-\frac{4 \sin ^{2} \omega \tau / 2}{\omega \tau}\right), \\
j_{1}(\tau)=\frac{u_{p}}{\omega}\left(\frac{2 \sin ^{2} \omega \tau / 2}{(\omega \tau)^{2}}-\frac{\sin \omega \tau}{\omega \tau}+\frac{\cos \omega \tau}{2}\right), \\
j_{2}(\tau)=\frac{u_{p}}{\omega}\left(\frac{2 \sin ^{2} \omega \tau / 2}{(\omega \tau)^{2}}-\frac{\sin \omega \tau}{\omega \tau}+\frac{1}{2}\right),
\end{gathered}
$$

where $u_{p}=F^{2}\left(2 \omega^{2}\right)^{-1}$.

(i) $s$ states [cf. Eq. (26)]:

$$
\begin{aligned}
M_{k, k^{\prime}}(\epsilon)= & \frac{i^{k-k^{\prime}}}{\sqrt{4 \pi i}} \int_{0}^{\infty} \frac{d \tau}{\tau^{3 / 2}} e^{i\left(\epsilon+\left(k+k^{\prime}\right) \omega\right) \tau} \\
& \times\left[e^{-i \lambda(\tau)} J_{k-k^{\prime}}(\ell z(\tau))-\delta_{k, k^{\prime}}\right] .
\end{aligned}
$$

(ii) $p$ states: elliptic polarization [cf. Eqs. (54) and (55)]:

$$
\begin{aligned}
M_{k, k^{\prime}}^{(0)}(\epsilon)= & \frac{3}{2} \frac{i^{k-k^{\prime}-1}}{\sqrt{4 \pi i}} \int_{0}^{\infty} e^{i\left[\epsilon+\left(k+k^{\prime}\right) \omega\right] \tau} \\
& \times \frac{\left\{e^{-i \lambda(\tau)} J_{k-k^{\prime}}(\ell z(\tau))-\delta_{k, k^{\prime}}\right\}}{\tau^{5 / 2}} d \tau,
\end{aligned}
$$

$$
\begin{aligned}
\bar{M}_{k, k^{\prime}}(\epsilon, \xi)= & M_{k, k^{\prime}}^{(0)}+3 \omega \frac{i^{k-k^{\prime}-1}}{\sqrt{4 \pi i}} \\
& \times \int_{0}^{\infty} \frac{e^{i\left[\epsilon+\left(k+k^{\prime}\right) \omega\right] \tau-i \lambda(\tau)}}{\tau^{3 / 2}}\left[\left(\frac{\xi}{2} z(\tau)+i j_{1}(\tau)\right)\right. \\
& \left.\times J_{k-k^{\prime}}(\ell z(\tau))-\ell J^{\prime}{ }_{k-k^{\prime}}(\ell z(\tau)) j_{2}(\tau)\right] d \tau
\end{aligned}
$$

$$
\begin{aligned}
\hat{M}_{k, k^{\prime}}(\epsilon, \xi)= & 3 \omega \frac{i^{k-k^{\prime}-1}}{\sqrt{4 \pi i}} \int_{0}^{\infty} \frac{e^{i\left[\epsilon+\left(k+k^{\prime}\right) \omega\right] \tau-i \lambda(\tau)}}{\tau^{3 / 2}} \\
& \times\left\{-i \ell J_{k-k^{\prime}}(\ell z(\tau)) j_{1}(\tau)\right. \\
& \left.+\left[J^{\prime}{ }_{k-k^{\prime}}(\ell z(\tau))+\xi \frac{k-k^{\prime}}{\ell z(\tau)} J_{k-k^{\prime}}(\ell z(\tau))\right] j_{2}(\tau)\right\} d \tau .
\end{aligned}
$$

(iii) $p$ states: linear polarization [cf. Eq. (38)]:

$$
\begin{gathered}
M_{k, k^{\prime}}(\epsilon)=M_{k, k^{\prime}}^{(0)}(\epsilon, \ell=1), \\
\tilde{M}_{k, k^{\prime}}(\epsilon)=\bar{M}_{k, k^{\prime}}(\epsilon, \xi=0)-M_{k, k^{\prime}}(\epsilon)-\hat{M}_{k, k^{\prime}}(\epsilon, \xi=0) .
\end{gathered}
$$

(iv) $p$ states: circular polarization [cf. Eq. (49)]:

$$
\begin{gathered}
\overline{\mathcal{M}}_{ \pm}(\epsilon+2 k \omega)=\bar{M}_{k, k}(\epsilon, \xi= \pm 1), \\
\hat{\mathcal{M}}(\epsilon+(2 k-1) \omega)=\hat{M}_{k, k-1}(\epsilon, \xi=1), \\
\hat{\mathcal{M}}(\epsilon+(2 k+1) \omega)=\hat{M}_{k, k+1}(\epsilon, \xi=-1) .
\end{gathered}
$$

As follows from the explicit forms of the matrix elements (B5)-(B8) [as well as of Eqs. (22), (30), and (47)], the integrals with respect to $\tau$ in these equations are formally divergent at the upper limit since $\operatorname{Im} \epsilon<0$; they are to be understood as the analytical continuation from the upper halfplane of complex $\epsilon$, where $\operatorname{Im} \epsilon>0$. Such divergencies are typical for the theory of quasistationary states and different methods for the numerical regularization of singular integrals in this theory can be used (such as, e.g., the "Zel'dovich regularization" $[59,60])$. However, for our purposes the most 
convenient method is to perform an analytic continuation procedure, as in Ref. [42] (see also Appendix B3 of Ref. [8]), using the relation

$$
\int_{0}^{\infty} \frac{e^{i \epsilon \tau}}{\tau^{1 / 2}} f(\tau) d \tau=\frac{1}{\sqrt{4 \pi i}} \int_{-\infty}^{\infty} \frac{d k}{\sqrt{\epsilon+k}} \int_{-\infty}^{\infty} d \tau e^{i k \tau} f(\tau),
$$

where the integral on the RHS is convergent for any complex $\epsilon$. The Fourier transform in Eq. (B11) can be calculated very efficiently using a fast Fourier transform algorithm, and then the integration over $k$ can also be performed numerically with high accuracy.

\section{APPENDIX C: ANALYTIC EXPLANATION OF THE ELLIPTICITY DEPENDENCE OF THE $p$-STATE FOURIER COEFFICIENTS $f_{k}^{( \pm 1)}\left(\epsilon_{+1}\right)$}

To explain the increase of the Fourier coefficients $f_{k}^{( \pm 1)}\left(\epsilon_{+1}\right)$ with increasing $\xi$ that is exhibited in Fig. 15, we transform the QQES wave functions (51) (which are defined in the reference frame $\{X, Y, Z\}$ whose $Z$ axis is directed along the wave vector of the laser field) to the "primed" reference frame $\left\{X^{\prime}, Y^{\prime}, Z^{\prime}\right\}$ whose quantization axis $Z^{\prime}$ is directed along the major axis of the polarization ellipse. As follows from the general form of the QQES wave function (18), which is an irreducible tensor of rank $l$, the functions $\widetilde{\Phi}^{(p=0, \pm 1)}$ in Eq. (52) transform similarly to spherical harmonics $Y_{1 p}(\hat{\mathbf{r}})$-i.e., with use of the Wigner functions $D_{p, p^{\prime}}^{j=1}(\alpha, \beta, \gamma)$, where the Euler angles in our case are $\alpha=0$, $\beta=\pi / 2$, and $\gamma=0$. Taking into account the explicit form of $D^{j=1}[35]$, we find that the wave functions (51) transform as follows:

$$
\begin{aligned}
\tilde{\Phi}^{(0)} \rightarrow & \frac{1}{\sqrt{2}}\left[\hat{\Phi}^{(+1)}-\hat{\Phi}^{\prime(-1)}\right] f^{(0)}(t), \\
{\left[\widetilde{\Phi}^{(+1)}+\widetilde{\Phi}^{(-1)}\right] \rightarrow } & \hat{\Phi}^{\prime(0)} \frac{f^{(+1)}(t)-f^{(-1)}(t)}{\sqrt{2}} \\
& +\left[\hat{\Phi}^{(+1)}+\hat{\Phi}^{(-1)}\right] \frac{f^{(+1)}(t)+f^{(-1)}(t)}{2},
\end{aligned}
$$

where the formal integral operator $\hat{\Phi}^{\left(m^{\prime}\right)}$, acting in the "primed" reference frame, is introduced, in agreement with the definition (18) for the QQES having $l=1-$ i.e.,

$$
\begin{aligned}
\hat{\Phi}^{(m)} f^{(p)}(t) \equiv & 4 \pi i C_{\kappa 1} \int_{-\infty}^{t} e^{i \epsilon\left(t-t^{\prime}\right)} G\left(\mathbf{r}, t ; 0, t^{\prime}\right) \\
& \times \mathcal{Y}_{1, m}\left(\frac{\mathbf{r}}{2\left(t-t^{\prime}\right)}+\boldsymbol{\alpha}\left(t, t^{\prime}\right)\right) f^{(p)}\left(t^{\prime}\right) d t^{\prime}
\end{aligned}
$$

Consider first the case of linear polarization $(\xi=0)$, for which the two-component solutions $\left\{f^{(+1)}(t), f^{(-1)}(t)\right\}$ of Eq. (55) reduce to the "symmetric" solution $\left\{f^{(+)}(t), f^{(+)}(t)\right\}$ with complex quasienergy $\epsilon_{+}$and the "antisymmetric" one $\left\{f^{(-)}(t),-f^{(-)}(t)\right\}$ with quasienergy $\epsilon_{-}$. In both cases the pair of functions $f^{( \pm 1)}(t)$ reduces to a single function, either $f^{(+)}(t)$ or $f^{(-)}(t)$. According to the transformation rule $(\mathrm{C} 2)$, the wave functions (51) with mixed projections $m= \pm 1$ on the $Z$ axis are represented in the "primed" reference frame as a superposition of functions $\Phi_{\epsilon_{\left|m^{\prime}\right|}, m^{\prime}}(\mathbf{r}, t)$ introduced for the case of linear polarization (cf. Sec. IV), where $m^{\prime}$ is the conserved projection of angular momentum on the direction of laser polarization (the $Z^{\prime}$ axis). For the "symmetric" solution of Eq. (55), the corresponding QQES wave function $\Phi_{\epsilon_{+}}^{s}(\mathbf{r}, t)$ is given by the symmetric superposition of the functions $\Phi_{\epsilon_{\mid m^{\prime}, m^{\prime}}}$ with $m^{\prime}= \pm 1$ [since the term $f^{(+1)}(t)-f^{(-1)}(t)$ in Eq. (C2) vanishes]. In contrast, the "antisymmetric" solution $\Phi_{\epsilon_{-}}^{a}(\mathbf{r}, t)$ coincides with $\Phi_{\epsilon_{\left|m^{\prime}\right| m^{\prime}}}(\mathbf{r}, t)$ for $m^{\prime}=0$ [since $f^{(+1)}(t)+f^{(-1)}(t)=0$ for this case]. Since the projection $m^{\prime}$ is a conserved quantum number for a linearly polarized field, the wave function $\Phi_{\epsilon}^{s}$ contains partial-wave components with $m^{\prime}=0$ (and thus with $l=0$ ), whereas the function $\Phi_{\epsilon_{+}}^{a}$ does not. The lack of angular momentum components with $l=0$ in the partial-wave expansion of $\Phi_{\epsilon_{+}}^{s}$ explains the smallness of the Fourier coefficients of $f^{(+)}(t)$ [or, equivalently, of the coefficients $f_{k}^{(-1)}\left(\epsilon_{+1}\right)$ in Fig. 15] as compared to those for $f^{(-)}(t)$ [or $f_{k}^{(-1)}\left(\epsilon_{-1}\right)$ in Fig. 15].

For the case of $\xi \neq 0$, the elliptically polarized field destroys the axial symmetry of the Hamiltonian in a linearly polarized field, so that the angular momentum projection $m^{\prime}$ is no longer a conserved quantum number of QQESs. Therefore, as $\xi$ increases from zero, the contribution of partial waves with $\left|m^{\prime}\right|>0$ (and thus $l>0$ ) to the QQES wave function corresponding to $\Phi_{\epsilon_{-}}^{a}(\mathbf{r}, t)$ at $\xi=0$ increases, while the Fourier coefficients $f_{k}^{( \pm 1)}\left(\epsilon_{-1}\right)$ begin decreasing (cf. Fig. 15). In contrast, the function $\Phi_{\epsilon_{+}}^{s}(\mathbf{r}, t)$ with increasing $\xi$ acquires partial-wave components with $m^{\prime}=0$, so that the coefficients $f_{k}^{( \pm 1)}\left(\epsilon_{+1}\right)$ start to grow (cf. Fig. 15). In fact, for $\xi>0$, each of the two solutions of Eq. (55) involves two different components $\left\{f^{(+1)}(t), f^{(-1)}(t)\right\}$. For the QQES having the quasienergy $\epsilon_{+1}$ [and corresponding to $\Phi_{\epsilon_{+}}^{s}(\mathbf{r}, t)$ at $\xi=0$ ], the population of field-induced components with $m^{\prime}=0$ is proportional to the difference $f^{+1}(t)-f^{-1}(t)$ [cf. Eq. (C2)], which increases with increasing $\xi$, starting from zero at $\xi$ $=0$, and leads to the increase of the Fourier coefficients $f_{k}^{( \pm 1)}\left(\epsilon_{+1}\right)$ of the functions $f^{( \pm 1)}(t)$ with increasing ellipticity. 
[1] K. J. Schafer, B. Yang, L. F. DiMauro, and K. C. Kulander, Phys. Rev. Lett. 70, 1599 (1993).

[2] P. B. Corkum, Phys. Rev. Lett. 71, 1994 (1993).

[3] P. Salières, A. L'Huillier, P. Antoine, and M. Lewenstein, Adv. At., Mol., Opt. Phys. 41, 83 (1999).

[4] W. Becker, F. Grasbon, R. Kopold, D. B. Milošević, G. G. Paulus, and H. Walther, Adv. At., Mol., Opt. Phys. 48, 35 (2002).

[5] N. L. Manakov, A. F. Starace, A. V. Flegel, and M. V. Frolov, Pis'ma Zh. Eksp. Teor. Fiz. 76, 316 (2002) [JETP Lett. 76, 258 (2002)].

[6] A. Čerkić and D. B. Milošević, Phys. Rev. A 70, 053402 (2004).

[7] A. V. Flegel, M. V. Frolov, N. L. Manakov, and A. F. Starace, Phys. Lett. A 334, 197 (2005).

[8] N. L. Manakov, M. V. Frolov, B. Borca, and A. F. Starace, J. Phys. B 36, R49 (2003).

[9] L. D. Landau and E. M. Lifshitz, Quantum Mechanics (Nonrelativistic Theory), 3rd ed. (Pergamon, Oxford, 1977).

[10] N. L. Manakov, V. D. Ovsiannikov, and L. P. Rapoport, Phys. Rep. 141, 319 (1986).

[11] M. V. Frolov, N. L. Manakov, E. A. Pronin, and A. F. Starace, Phys. Rev. Lett. 91, 053003 (2003).

[12] M. V. Frolov, N. L. Manakov, and A. F. Starace, J. Phys. B 36, L419 (2003).

[13] I. Yu. Kiyan and H. Helm, Phys. Rev. Lett. 90, 183001 (2003).

[14] M. V. Frolov, A. V. Flegel, N. L. Manakov, and A. F. Starace, J. Phys. B 38, L375 (2005).

[15] M. V. Frolov, A. A. Khuskivadze, N. L. Manakov, and A. F. Starace, J. Phys. B 39, S283 (2006).

[16] N. L. Manakov and M. V. Frolov, Pis'ma Zh. Eksp. Teor. Fiz. 83, 630 (2006) [JETP Lett. 83, 536 (2006)].

[17] M. V. Frolov, A. V. Flegel, N. L. Manakov, and A. F. Starace, Phys. Rev. A 75, 063408 (2007).

[18] N. L. Manakov, A. F. Starace, A. V. Flegel, and M. V. Frolov, Pis'ma Zh. Eksp. Teor. Fiz. 87, 99 (2008) [JETP Lett. 87, 92 (2008)].

[19] M. V. Frolov, N. L. Manakov, and A. F. Starace, Phys. Rev. Lett. 100, 173001 (2008).

[20] Yu. N. Demkov and V. N. Ostrovsky, Zero-Range Potentials and Their Applications in Atomic Physics (Plenum, New York, 1988).

[21] N. L. Manakov and L. P. Rapoport, Zh. Eksp. Teor. Fiz. 69, 842 (1975) [Sov. Phys. JETP 42, 430 (1975)].

[22] I. J. Berson, J. Phys. B 8, 3078 (1975).

[23] N. L. Manakov and A. G. Fainshtein, Zh. Eksp. Teor. Fiz. 79, 751 (1980) [Sov. Phys. JETP 52, 382 (1980)].

[24] W. Becker, S. Long, and J. K. McIver, Phys. Rev. A 50, 1540 (1994).

[25] N. L. Manakov, M. V. Frolov, A. F. Starace, and I. I. Fabrikant, J. Phys. B 33, R141 (2000).

[26] D. B. Milošević, A. Gazibegović-Busuladžić, and W. Becker, Phys. Rev. A 68, 050702(R) (2003).

[27] K. Krajewska, I. I. Fabrikant, and A. F. Starace, Phys. Rev. A 74, 053407 (2006).
[28] V. B. Berestetskii, E. M. Lifshitz, and L. P. Pitaevskii, Quantum Electrodynamics, 2nd ed. (Pergamon, Oxford, 1992), Sec. 8.

[29] M. V. Frolov, A. V. Flegel, N. L. Manakov, and A. F. Starace, Phys. Rev. A 75, 063407 (2007).

[30] Yu. N. Demkov and G. F. Drukarev, Zh. Eksp. Teor. Fiz. 81, 1218 (1981) [Sov. Phys. JETP 54, 650 (1981)].

[31] S. P. Andreev, B. M. Karnakov, and V. D. Mur, Pis'ma Zh. Eksp. Teor. Fiz. 37, 155 (1983) [JETP Lett. 37, 187 (1983)]; S. P. Andreev, B. M. Karnakov, V. D. Mur, and V. A. Polunin, Zh. Eksp. Teor. Fiz. 86, 866 (1984) [Sov. Phys. JETP 59, 506 (1984)].

[32] S. P. Andreev, B. M. Karnakov, and V. D. Mur, Teor. Mat. Fiz. 64, 287 (1985) [Theor. Math. Phys. 64, 838 (1985)].

[33] A. I. Baz', Ya. B. Zel'dovich, and A. M. Perelomov, Scattering, Reactions and Decays in Nonrelativistic Quantum Mechanics, 2nd ed. (Nauka, Moscow, 1971).

[34] N. L. Manakov, S. I. Marmo, and A. V. Meremianin, J. Phys. B 29, 2711 (1996).

[35] D. A. Varshalovich, A. N. Moskalev, and V. K. Khersonskii, Quantum Theory of Angular Momentum (World Scientific, Singapore, 1988).

[36] See Problem 3 at the end of Sec. 133 in Ref. [9].

[37] T. Ohmura and H. Ohmura, Phys. Rev. 118, 154 (1960).

[38] M. L. Du and J. B. Delos, Phys. Rev. A 38, 5609 (1988).

[39] V. D. Mur, S. V. Popruzhenko, S. G. Pozdnyakov, and V. S. Popov, Phys. Lett. A 316, 226 (2003).

[40] A. A. Radzig and B. M. Smirnov, Reference Data on Atoms, Molecules and Ions (Springer, Berlin, 1985).

[41] M. V. Frolov, N. L. Manakov, B. Borca, and A. F. Starace, J. Phys. B 34, L579 (2001).

[42] N. L. Manakov, M. V. Frolov, B. Borca, and A. F. Starace, Pis'ma Zh. Eksp. Teor. Fiz. 72, 426 (2000) [JETP Lett. 72, 294 (2000)].

[43] E. P. Wigner, Phys. Rev. 73, 1002 (1948).

[44] D. A. Telnov and Shin-I Chu, J. Phys. B 37, 1489 (2004).

[45] L. V. Keldysh, Zh. Eksp. Teor. Fiz. 47, 1945 (1964) [Sov. Phys. JETP 20, 1307 (1965)].

[46] G. F. Gribakin and M. Yu. Kuchiev, Phys. Rev. A 55, 3760 (1997)

[47] A. M. Perelomov, V. S. Popov, and M. V. Terent'ev, Zh. Eksp. Teor. Fiz. 50, 1393 (1966) [Sov. Phys. JETP 23, 924 (1966)].

[48] A. V. Flegel, M. V. Frolov, N. L. Manakov, and A. F. Starace, J. Phys. B 38, L27 (2005).

[49] A. M. Perelomov, V. S. Popov, and M. V. Terent'ev, Zh. Eksp. Teor. Fiz. 51, 309 (1966) [Sov. Phys. JETP 24, 207 (1967)].

[50] J. Pedregosa-Gutierrez, P. A. Orr, J. B. Greenwood, A. Murphy, J. T. Costello, K. Zrost, T. Ergler, R. Moshammer, and J. Ullrich, Phys. Rev. Lett. 93, 223001 (2004).

[51] B. Bergues, Y. Ni, H. Helm, and I. Yu. Kiyan, Phys. Rev. Lett. 95, 263002 (2005).

[52] B. Bergues, Z. Ansari, D. Hanstorp, and I. Yu. Kiyan, Phys. Rev. A 75, 063415 (2007).

[53] N. L. Manakov and A. G. Fainshtein, Dokl. Akad. Nauk SSSR 244, 567 (1979) [Sov. Phys. Dokl. 24, 41 (1979)]. 
[54] S. Beiser, M. Klaiber, and I. Yu. Kiyan, Phys. Rev. A 70, 011402(R) (2004).

[55] R. M. Potvliege, Phys. Rev. A 62, 013403 (2000).

[56] R. Bhatt, B. Piraux, and K. Burnett, Phys. Rev. A 37, 98 (1988).

[57] Handbook of Mathematical Functions, edited by M. Abramowitz and I. A. Stegun (Dover, New York, 1995).
[58] N. L. Manakov, A. V. Meremianin, and A. F. Starace, Phys. Rev. A 57, 3233 (1998).

[59] Ya. B. Zel'dovich, Zh. Eksp. Teor. Fiz. 39, 776 (1960) [Sov. Phys. JETP 12, 542 (1961)].

[60] V. D. Mur, S. G. Pozdnyakov, S. V. Popruzhenko, and V. S. Popov, Phys. At. Nucl. 68, 677 (2005). 